\title{
ON DEGENERATE INTERPOLATION, ENTROPY AND EXTREMAL PROBLEMS FOR MATRIX SCHUR FUNCTIONS
}

\author{
VLADIMIR BOLOTNIKOV and HARRY DYM *
}

\begin{abstract}
We consider a general bitangential interpolation problem for matrix Schur functions and focus mainly on the case when the associated Pick matrix is singular (and positive semidefinite). Descriptions of the set of all solutions are given in terms of special linear fractional transformations which are obtained using two quite different approaches. As applications of the obtained results we consider the maximum entropy and the maximum determinant extension problems suitably adapted to the degenerate situation.
\end{abstract}

\section{Introduction}

This paper is devoted to three main themes. The first and major theme is a study of representation formulas for bitangential matrix problems in the Schur class with a finite number of interpolation constraints when the associated Pick matrix is singular. The second two themes are applications of these representation formulas to appropriate versions of a maximum entropy problem and a maximum determinant extension problems in the singular case. We shall work within the framework of the augmented Basic Interpolation Problem. An introduction to this problem, which includes an account of its development from more elementary problems as well as other formulations, appears in [16].

In order to describe the Basic Interpolation Problem (BIP) we need to introduce some notation. Let $\mathbf{H}_{2}^{p \times q}$ denotes the set of $\mathbb{C}^{p \times q}$-valued functions with entries in the Hardy space $\mathbf{H}_{2}$ of the unit disc $\mathbb{D}$ and let $\mathbf{H}_{2}^{k \times 1}$ be abbreviated by $\mathbf{H}_{2}^{k}$. Similarly, let $L_{2}^{k}(\mathbb{T})$ designate the Hilbert space of measurable and square integrable $\mathbb{C}^{k}$-valued functions with inner product

$$
\langle f, g\rangle=\frac{1}{2 \pi} \int_{0}^{2 \pi} g\left(e^{i t}\right)^{*} f\left(e^{i t}\right) d t, \quad\left(f, g \in L_{2}^{k}(\mathbb{T})\right) .
$$

The space $\mathbf{H}_{2}^{k}$ is identified as the closed subspace of $L_{2}^{k}(\mathbb{T})$ which consists of all $f \in L_{2}^{k}(\mathbb{T})$ whose negative Fourier coefficients are equal to zero. The symbol $\mathbf{H}_{2}^{k \perp}$ stands for the orthogonal complement of $\mathbf{H}_{2}^{k}$ with respect to the inner product (1.1). More generally, $\mathbf{H}_{2}^{p \times q^{\perp}}$

\footnotetext{
${ }^{*}$ H. Dym would like to thank Renee and Jay Weiss for endowing the chair which supports his research.
} 
denotes the set of $\mathbb{C}^{p \times q}$-valued functions with entries in $\mathbf{H}_{2}{ }^{\perp}$. The Schur class of $\mathbb{C}^{p \times q}$ valued analytic contractions in $\mathbb{D}$ is denoted by $\mathcal{S}^{p \times q}$. Throughout the paper $J$ denotes the signature matrix defined by

$$
J=\left(\begin{array}{cc}
I_{p} & 0 \\
0 & -I_{q}
\end{array}\right)
$$

where $I_{q}$ stands for the identity matrix in $\mathbb{C}^{q \times q}$.

Let

$$
M, N, P \in \mathbb{C}^{n \times n} \text { and } C \in \mathbb{C}^{(p+q) \times n}
$$

be a given set of matrices and let $\operatorname{aBIP}(M, N, P, C)$ denote the following "augmented basic interpolation problem" which is now formulated under the assumption that the mvf (matrix valued function)

$$
G(z)=M-z N
$$

is invertible at every point on the unit circle:

$$
\operatorname{det} G(\zeta) \neq 0 \text { for }|\zeta|=1 \text {. }
$$

1. Find necessary and sufficient conditions which ensure the existence of a Schur function $S \in \mathcal{S}^{p \times q}$ such that

$$
\left(\begin{array}{cc}
I_{p} & -S(\zeta) \\
-S(\zeta)^{*} & I_{q}
\end{array}\right) C G(\zeta)^{-1} \in\left(\begin{array}{c}
\mathbf{H}_{2}^{p \times n} \\
\left(\mathbf{H}_{2}^{q \times n}\right)^{\perp}
\end{array}\right)
$$

and

$$
\left\langle\left(\begin{array}{cc}
I_{p} & -S(\zeta) \\
-S(\zeta)^{*} & I_{q}
\end{array}\right) C G(\zeta)^{-1} y, C G(\zeta)^{-1} x\right\rangle_{L_{2}^{p+q}(\mathbb{T})}=x^{*} P y \quad\left(\forall x, y \in \mathbb{C}^{n}\right)
$$

2. Describe the set of all such functions.

The solvability criterion of the $\operatorname{aBIP}(M, N, P, C)$ is easily stated:

Theorem 1.1 Let (1.5) be in force. Then the $\operatorname{aBIP}(M, N, P, C)$ has a solution if and only if $P$ is a nonnegative solution of the Lyapunov-Stein equation

$$
M^{*} P M-N^{*} P N=C^{*} J C .
$$

The proof of necessity reduces to a straightforward calculation which is reviewed in Section 2. The asserted sufficiency is an immediate consequence of the next theorem which will be established in Section 7. The partition

$$
C=\left(\begin{array}{l}
C_{1} \\
C_{2}
\end{array}\right), \quad C_{1} \in \mathbb{C}^{p \times n}, \quad C_{2} \in \mathbb{C}^{q \times n},
$$

which enables us to express the Lyapunov-Stein equation (1.8) in the form

$$
M^{*} P M-N^{*} P N=C_{1}^{*} C_{1}-C_{2}^{*} C_{2},
$$

will be useful. 
Theorem 1.2 Let (1.5) be in force, let $P$ be a nonnegative solution of the Lyapunov-Stein equation (1.8) and let

$$
\nu=\operatorname{rank}\left(M^{*} P M+C_{2}^{*} C_{2}\right)-\operatorname{rank} P=\operatorname{rank}\left(N^{*} P N+C_{1}^{*} C_{1}\right)-\operatorname{rank} P .
$$

Then

$$
0 \leq \nu \leq \min (p, q)
$$

and there exists a rational $(p+q) \times(p+q) \operatorname{mvf} \boldsymbol{\Theta}(z)$,

$$
\boldsymbol{\Theta}=\left(\begin{array}{ll}
\Theta_{11} & \Theta_{12} \\
\Theta_{21} & \Theta_{22}
\end{array}\right):\left(\begin{array}{l}
\mathbb{C}^{p} \\
\mathbb{C}^{q}
\end{array}\right) \rightarrow\left(\begin{array}{l}
\mathbb{C}^{p} \\
\mathbb{C}^{q}
\end{array}\right)
$$

which is J-inner in $\mathbb{D}$ and defines a one to one map via the linear fractional transformation

$$
S(z)=\left(\boldsymbol{\Theta}_{11}(z) \mathcal{E}(z)+\boldsymbol{\Theta}_{12}(z)\right)\left(\boldsymbol{\Theta}_{21}(z) \mathcal{E}(z)+\boldsymbol{\Theta}_{22}(z)\right)^{-1}
$$

from the set of all $\mathcal{E} \in \mathcal{S}^{p \times q}$ of the form

$$
\mathcal{E}(z)=\left(\begin{array}{cc}
\widehat{\mathcal{E}}(z) & 0 \\
0 & I_{\nu}
\end{array}\right), \quad \widehat{\mathcal{E}} \in \mathcal{S}^{(p-\nu) \times(q-\nu)},
$$

onto the set of all solutions $S(z)$ of the $\operatorname{aBIP}(M, N, P, C)$. Moreover, the McMillan degree of $\Theta(z)$ is equal to the rank of $P$.

In other words, a mvf $S$ is a solution of the $\operatorname{aBIP}(M, N, P, C)$ if and only if it admits a representation (1.13) for some (uniquely defined) parameter $\mathcal{E}$ of the form (1.14).

The $\operatorname{aBIP}(M, N, P, C)$ is termed nondegenerate if $P>0$ and is termed degenerate if $P$ is singular (and positive semidefinite).

Let us mention two particular cases of Theorem 1.2 corresponding to the extreme values of $\nu$. The first one is well known. If $P$ is invertible, then $\nu=0$ and the set of all solutions of the nondegenerate aBIP is parametrized by the linear fractional transformation (1.13) when the parameter $\mathcal{E}$ varies over all of $\mathcal{S}^{p \times q}$.

In the opposite extreme case when $\nu=\min (p, q)$ we conclude from (1.14) that the parameter $\mathcal{E}$ is constant:

$$
\mathcal{E}(z) \equiv\left(\begin{array}{ll}
0 & I_{p}
\end{array}\right) \text { if } p \leq q \quad \text { and } \quad \mathcal{E}(z) \equiv\left(\begin{array}{c}
0 \\
I_{q}
\end{array}\right) \text { if } p \geq q,
$$

and so, by Theorem 1.2, the aBIP has a unique solution. Thus, we obtain the following simple criterion of uniqueness:

Theorem 1.3 Let (1.5) be in force, let $P$ be a nonnegative solution of the Lyapunov-Stein equation (1.8). Then the $\operatorname{aBIP}(M, N, P, C)$ has a unique solution if and only if

$$
\operatorname{rank}\left(M^{*} P M+C_{2}^{*} C_{2}\right)-\operatorname{rank} P=\min (p, q) .
$$


The latter situation clearly can happen only if $P$ is singular. Degenerate cases of the classical Nevanlinna-Pick and Carathéodory-Fejér interpolation problems for Schur functions were studied using quite different approaches in [9], [10], [11] and [13, Section 7]. The results obtained below in Section 7 rely on Potapov's method (which characterizes the solutions of an interpolation problem in terms of a related fundamental matrix inequality suitably adapted to the present framework; for some early examples of this method, see [27]).

In Section 8 we present another description of all the solutions of the aBIP in terms of the Redheffer transformation

$$
S(z)=\Psi_{12}(z)+\Psi_{11}(z) \widehat{\mathcal{E}}(z)\left(I_{q-\nu}-\Psi_{21}(z) \widehat{\mathcal{E}}(z)\right)^{-1} \Psi_{22}(z)
$$

where the $\Psi_{j k}(z)$ are obtained from the $\theta_{j k}(z)$ by the Potapov-Ginzburg transform and $\widehat{\mathcal{E}}$ is a free parameter varying over all of $\mathcal{S}^{(p-\nu) \times(q-\nu)}$. In Section 9 we obtain a Redheffer description of all the solutions of the aBIP using quite a different approach which is based on identifying the set of solutions to the aBIP with the characteristic functions of unitary colligations which extend an isometric operator constructed in a natural way from the data of the problem. This approach is adapted from the work of Katsnelson, Kheifets and Yuditskii [23] on the abstract interpolation problem and the works of Arov and Grossman [?], [?] on the coupling of open systems. Explicit formulas for the coefficients $\Psi_{j k}$ are given in Section 10 and are then applied in Sections 11 and 12 to solve two extremal problems for Schur functions. The first one consists of finding the solution $S$ of the aBIP, which maximizes an entropy integral. The second is a maximum determinant extension problem for structured matrices which is formulated in purely algebraic language, but is solved using analytic methods. This is a generalization of results from [13], [15] and [17], where the above extremal problems were considered for the nondegenerate situation. In Section 13 we discuss the aBIP for analytic contractions defined on a general class of domains.

We shall make frequent use of the mvf

$$
H(z)=z M^{*}-N^{*}
$$

and the notations

$$
\rho_{\omega}(z)=1-z \bar{\omega} \quad \text { and } \quad \delta_{\omega}(z)=z-\omega .
$$

In this paper we shall focus on the case when $G$ is invertible on $\mathbb{T}$. However, for ease of future reference, we shall carry out parts of the analysis under the less restrictive assumption that

$$
\operatorname{det} G(z) \not \equiv 0 \text {. }
$$

For the case when $G$ is invertible on $\mathbb{T}$, the interpolation conditions of the aBIP can be expressed in terms of contour integrals. The formulation of one sided interpolation problems in terms of contour integrals was suggested by A. Nudelman in [?] and extended to two sided problems in [8], where a full description of the set of all solutions is given for the case when the associated Pick matrix $P$ is invertible; for additional discussion and comparison, see [14, Section 7.4]. 


\section{Preliminary analysis of the problem}

Let $[,]_{S}$ be the matrix valued Hermitian form which is associated with $S \in \mathcal{S}^{p \times q}$ by the rule

$$
[h, g]_{S}=\frac{1}{2 \pi} \int_{0}^{2 \pi} g\left(e^{i t}\right)^{*}\left(\begin{array}{cc}
I_{p} & -S\left(e^{i t}\right) \\
-S\left(e^{i t}\right)^{*} & I_{q}
\end{array}\right) h\left(e^{i t}\right) d t
$$

for every pair $h \in L_{2}^{(p+q) \times k}(\mathbb{T})$ and $g \in L_{2}^{(p+q) \times \ell}$ and any positive integers $k$ and $\ell$.

Lemma 2.1 Let $S \in \mathcal{S}^{p \times q}$ satisfy the condition (1.6). Then the matrix

$$
P_{S}:=\left[C G(\zeta)^{-1}, C G(\zeta)^{-1}\right]_{S}
$$

is nonnegative and satisfies the Lyapunov-Stein equation

$$
M^{*} P_{S} M-N^{*} P_{S} N=C_{1}^{*} C_{1}-C_{2}^{*} C_{2} .
$$

Proof: If $S \in \mathcal{S}^{p \times q}$, then

$$
\left(\begin{array}{cc}
I_{p} & -S(\zeta) \\
-S(\zeta)^{*} & I_{q}
\end{array}\right) \geq 0 \quad(\text { for a.e. } \zeta \in \mathbb{T})
$$

which implies that the form $(2.1)$ is nonnegative. In particular, the matrix $P_{S}$ defined by (2.2) is nonnegative. In view of (1.4),

$$
\zeta G(\zeta)^{-1} N=G(\zeta)^{-1} M-I_{n}
$$

and therefore,

$$
\begin{aligned}
& M^{*}\left[C G(\zeta)^{-1}, C G(\zeta)^{-1}\right]_{S} M-N^{*}\left[C G(\zeta)^{-1}, C G(\zeta)^{-1}\right]_{S} N \\
& =\left[C G(\zeta)^{-1} M, C G(\zeta)^{-1} M\right]_{S}-\left[C G(\zeta)^{-1} N, C G(\zeta)^{-1} N\right]_{S} \\
& =\left[C G(\zeta)^{-1} M, C G(\zeta)^{-1} M\right]_{S}-\left[C\left(G(\zeta)^{-1} M-I_{n}\right), C\left(G(\zeta)^{-1} M-I_{n}\right)\right]_{S} \\
& =\left[C G(\zeta)^{-1} M, C\right]_{S}+\left[C, C G(\zeta)^{-1} M\right]_{S}-[C, C]_{S} .
\end{aligned}
$$

But in view of (1.6),

$$
\begin{aligned}
{\left[C G(\zeta)^{-1} M, C\right]_{S}=} & \left\langle\left\{C_{1}-S(\zeta) C_{2}\right\} G(\zeta)^{-1} M, C_{1}\right\rangle_{L_{2}^{p}(\mathbb{T})} \\
& +\left\langle\left\{C_{2}-S(\zeta)^{*} C_{1}\right\} G(\zeta)^{-1} M, C_{2}\right\rangle_{L_{2}^{q}(\mathbb{T})} \\
= & \left\langle\left\{C_{1}-S(\zeta) C_{2}\right\} G(\zeta)^{-1} M, C_{1}\right\rangle_{L_{2}^{p}(\mathbb{T})}+0 \\
= & \left\langle\left\{C_{1}-S(\zeta) C_{2}\right\} G(\zeta)^{-1}(M-\zeta N), C_{1}\right\rangle_{L_{2}^{p}(\mathbb{T})} \\
= & \left\langle C_{1}-S(\zeta) C_{2}, C_{1}\right\rangle_{L_{2}^{p}(\mathbb{T})},
\end{aligned}
$$


whereas

$$
\left[C, C G(\zeta)^{-1} M\right]_{S}=\left\langle C_{1}, C_{1}-S(\zeta) C_{2}\right\rangle_{L_{2}^{p}(\mathbb{T})}
$$

and

$$
[C, C]_{S}=\left\langle C_{1}-S(\zeta) C_{2}, C_{1}\right\rangle_{L_{2}^{p}(\mathbb{T})}+\left\langle C_{2}-S(\zeta)^{*} C_{1}, C_{2}\right\rangle_{L_{2}^{q}(\mathbb{T})}
$$

Formula (2.3) now emerges easily from (2.6) upon substituting the preceding three evaluations.

As a corollary we obtain the necessity part of Theorem 1.1: if $S$ satisfies the conditions (1.6) and (1.7), then the Pick matrix $P$ is necessarily nonnegative and satisfies the LyapunovStein equation (1.8).

We shall say that two aBIP's are equivalent if they have the same set of solutions. The following simple observation is useful.

Lemma 2.2 Let $T_{1}$ and $T_{2}$ be two invertible $n \times n$ matrices and let

$$
\widehat{M}=T_{1} M T_{2}, \quad \widehat{N}=T_{1} N T_{2}, \quad \widehat{P}=T_{1}^{-*} P T_{1}^{-1}, \quad \text { and } \quad \widehat{C}=C T_{2} .
$$

Then the problems $\mathbf{a B I P}(M, N, P, C)$ and $\mathbf{a B I P}(\widehat{M}, \widehat{N}, \widehat{P}, \widehat{C})$ are equivalent.

Proof: It suffices to note that the matrices from (2.7) satisfies the equality

$$
\widehat{M}^{*} \widehat{P} \widehat{M}-\widehat{N}^{*} \widehat{P} \widehat{N}=\widehat{C}^{*} J \widehat{C}
$$

and that

$$
\widehat{C} \widehat{G}(z)^{-1}:=\left(\begin{array}{c}
\widehat{C}_{1} \\
\widehat{C}_{2}
\end{array}\right)(\widehat{M}-z \widehat{N})^{-1}=\left(\begin{array}{c}
C_{1} \\
C_{2}
\end{array}\right)(M-z N)^{-1} T_{1}^{-1}=C G(z)^{-1} T_{1}^{-1},
$$

which implies

$$
\left[\widehat{C} \widehat{G}(\zeta)^{-1}, \widehat{C} \widehat{G}(\zeta)^{-1}\right]_{S}=\left[C G(\zeta)^{-1} T_{1}^{-1}, C G(\zeta)^{-1} T_{1}^{-1}\right]_{S}=T_{1}^{-*} P T_{1}^{-1}=\widehat{P} .
$$

The next lemma allows us to express $G(z)$ in a certain canonical form which will simplify some later computations.

Lemma 2.3 Let $M$ and $N$ satisfy (1.17). Then there exist invertible matrices $T_{1}$ and $T_{2}$ from $\mathbb{C}^{n \times n}$ and matrices $A_{1} \in \mathbb{C}^{k_{1} \times k_{1}}, A_{2} \in \mathbb{C}^{k_{2} \times k_{2}}$ and $A_{3} \in \mathbb{C}^{\left(n-k_{1}-k_{2}\right) \times\left(n-k_{1}-k_{2}\right)}$ with

$$
\operatorname{spec} A_{1} \bigcup \operatorname{spec} A_{2} \subset \mathbb{D} \quad \text { and } \quad \operatorname{spec} A_{3} \subset \mathbb{T}
$$

such that

$$
T_{1} M T_{2}=\left(\begin{array}{ccc}
I_{k_{1}} & 0 & 0 \\
0 & A_{2} & 0 \\
0 & 0 & A_{3}
\end{array}\right) \quad \text { and } \quad T_{1} N T_{2}=\left(\begin{array}{ccl}
A_{1} & 0 & 0 \\
0 & I_{k_{2}} & 0 \\
0 & 0 & I_{n-k_{1}-k_{2}}
\end{array}\right)
$$


Proof: By (1.17), $G(z)$ is a regular pencil and therefore (see e.g., [18, p. 28, Theorem 3]), there exist a pair of invertible matrices $L_{1}$ and $L_{2}$ such that

$$
L_{1} M L_{2}=\left(\begin{array}{cc}
I_{r} & 0 \\
0 & \tilde{M}
\end{array}\right) \quad \text { and } \quad L_{1} N L_{2}=\left(\begin{array}{cc}
\tilde{N} & 0 \\
0 & I_{n-r}
\end{array}\right)
$$

where the matrices $\tilde{M}$ and $\tilde{N}$ are in Jordan form and are such that

$$
\operatorname{det} \tilde{M} \neq 0 \quad \text { and } \quad \operatorname{spec} \tilde{N}=\{0\}
$$

Without loss of generality, $\tilde{M}$ can be assumed to be of the following block diagonal form

$$
\tilde{M}=\operatorname{diag}\left\{M_{1}, M_{2}, M_{3}\right\}
$$

where

$$
\operatorname{spec} M_{1} \subset \mathbb{C} \backslash \overline{\mathbb{D}}, \quad \text { spec } M_{2} \subset \mathbb{D} \quad \text { and } \quad \operatorname{spec} M_{3} \subset \mathbb{T}
$$

and all of the blocks $\tilde{M}_{j}$ are of Jordan form. Then spec $M_{1}^{-1} \in \mathbb{D}$ and setting

$$
T_{1}=L_{1}, \quad T_{2}=L_{2} \operatorname{diag}\left\{I_{r} ; \tilde{M}_{1}^{-1} ; I_{\ell}\right\}
$$

and

$$
A_{1}=\operatorname{diag}\left\{\tilde{N} ; M_{1}^{-1}\right\}, \quad A_{2}=M_{2}, \quad A_{3}=M_{3},
$$

we get the required assertion.

Corollary 2.4 Let condition (1.5) be in force. Then, without loss of generality, the matrices $M$ and $N$ from the data set (1.3) of the $\mathbf{a B I P}$ can be assumed to be of the form

$$
M=\left(\begin{array}{cc}
I_{k} & 0 \\
0 & A_{2}
\end{array}\right), \quad N=\left(\begin{array}{cc}
A_{1} & 0 \\
0 & I_{n-k}
\end{array}\right)
$$

where $A_{1}$ and $A_{2}$ are matrices satisfying (2.8) and can be presumed to be in Jordan form.

Proof: In view of (1.5), the pencil $M-z N$ has no singular points on $\mathbb{T}$ and therefore, the matrix $A_{3}$ does not appear in representation (2.9). The rest follows from Lemma 2.2.

\section{A fundamental matrix inequality}

In this section we establish a number of equivalent conditions for a function $S \in \mathcal{S}^{q \times p}$ to be a solution of the aBIP. We recall that in this paper the aBIP is formulated under the assumption that condition (1.5) is in force. Nevertheless, for future convenience, some results in this section are derived under the less restrictive condition (1.17). The following notations will be useful. 
Definition 3.1 Let $\mathcal{S}(M, N, P, C)$ denote the set of all functions $S \in \mathcal{S}^{q \times p}$ which satisfy conditions (1.6) and (1.7). Let $\widehat{\mathcal{S}}(M, N, P, C)$ denote the set of all functions $S \in \mathcal{S}^{q \times p}$ which satisfy condition (1.6) and the following inequality:

$$
\left\langle\left(\begin{array}{cc}
I_{p} & -S(\zeta) \\
-S(\zeta)^{*} & I_{q}
\end{array}\right) C G(\zeta)^{-1} x, C G(\zeta)^{-1} x\right\rangle_{L_{2}^{p+q}(\mathbb{T})} \leq x^{*} P x \quad\left(\forall x \in \mathbb{C}^{n}\right) .
$$

The set $\mathcal{S}(M, N, P, C)$ coincides with the set of all solutions of the $\operatorname{aBIP}(M, N, P, C)$ and $\mathcal{S}(M, N, P, C) \subseteq \widehat{\mathcal{S}}(M, N, P, C)$. Sufficient conditions for the equality of these two sets are known; see e.g., [16, Lemma 6.1], from where the next lemma is adapted.

Lemma 3.2 Let P satisfy the Lyapunov-Stein equation (1.8) and let (1.5) hold. Then

$$
\mathcal{S}(M, N, P, C)=\widehat{\mathcal{S}}(M, N, P, C) .
$$

Proof: By Corollary 2.4, we may assume without loss of generality that the matrices $M$ and $N$ are of the form (2.10) for some choice of $A_{1}$ and $A_{2}$ satisfying the spectral condition (2.8). Let $S \in \widehat{\mathcal{S}}(M, N, P, C)$. Then $P_{S} \leq P$. By Lemma 2.1, $P_{S}$ satisfies the Lyapunov-Stein equation (2.3). Therefore, the nonnegative matrix

$$
\widehat{P}=\left(\begin{array}{ll}
\widehat{P}_{11} & \widehat{P}_{12} \\
\widehat{P}_{12}^{*} & \widehat{P}_{22}
\end{array}\right):=P-P_{S} \geq 0
$$

satisfies the homogeneous equation

$$
M^{*} \widehat{P} M-N^{*} \widehat{P} N=0 .
$$

The partition of $\widehat{P}$ in (3.2) corresponds to the partitions of $M$ and $N$ in (2.10). Substituting (2.10) and (3.2) into (3.3) we conclude in particular that the blocks $\widehat{P}_{11}$ and $\widehat{P}_{22}$ satisfy the homogeneous Stein equations

$$
\widehat{P}_{11}-A_{1}^{*} \widehat{P}_{11} A_{1}=0 \text { and } \widehat{P}_{22}-A_{2}^{*} \widehat{P}_{22} A_{2}=0,
$$

and hence, because of the spectral condition (2.8), that $\widehat{P}_{11}=0$ and $\widehat{P}_{22}=0$. Since the diagonal blocks of the nonnegative matrix $\widehat{P}$ are zero matrices, we also have $\widehat{P}_{12}=0$. Thus $\widehat{P}=0$, i.e., $P_{S}=P$ and $S \in \mathcal{S}(M, N, P, C)$. This proves the inclusion $\widehat{\mathcal{S}}(M, N, P, C) \subseteq$ $\mathcal{S}(M, N, P, C)$. Since the converse inclusion is clear, this finishes the proof of lemma.

The next lemma is the first step in characterizing all solutions of the aBIP in terms of the so called fundamental matrix inequality (FMI).

Lemma 3.3 Let $S$ belong to $\widehat{\mathcal{S}}(M, N, P, C)$. Then the inequality

$$
\left(\begin{array}{cc}
G(z)^{*} P G(z) & C^{*}\left(\begin{array}{c}
I_{p} \\
-S(z)^{*}
\end{array}\right) \\
\left(I_{p},-S(z)\right) C & \frac{I_{p}-S(z) S(z)^{*}}{\rho_{z}(z)}
\end{array}\right) \geq 0
$$

holds for every $z \in \mathbb{D}$. 
Proof: Let $S$ belong to $\widehat{\mathcal{S}}(M, N, P, C)$ (this means in particular, that $S \in \mathcal{S}^{p \times q}$ ) and let $[,]_{S}$ be the nonnegative Hermitian form defined via (2.1). Then,

$$
\left(\begin{array}{ll}
{\left[f_{1}, f_{1}\right]_{S}} & {\left[f_{2}, f_{1}\right]_{S}} \\
{\left[f_{1}, f_{2}\right]_{S}} & {\left[f_{2}, f_{2}\right]_{S}}
\end{array}\right) \geq 0 \quad\left(\forall f_{1}, f_{2} \in L_{2}^{p+q}(\mathbb{T})\right) .
$$

Fixing $x \in \mathbb{C}^{n}, y \in \mathbb{C}^{p}$ and a point $z \in \mathbb{D}$ at which $G(z)$ is invertible, set

$$
f_{1}(\zeta)=C G(\zeta)^{-1} x, \quad \text { and } \quad f_{2}(\zeta)=\left(\begin{array}{c}
I_{p} \\
S(z)^{*}
\end{array}\right) \frac{y}{\rho_{z}(\zeta)}
$$

in (3.5). Since $f_{2} \in \mathbf{H}_{2}^{p+q}$,

$$
\begin{aligned}
{\left[f_{2}, f_{2}\right]_{S} } & =\left\langle\left(\begin{array}{cc}
I_{p} & -S(\zeta) \\
-S(\zeta)^{*} & I_{q}
\end{array}\right)\left(\begin{array}{c}
I_{p} \\
S(z)^{*}
\end{array}\right) \frac{y}{\rho_{z}(\zeta)},\left(\begin{array}{c}
I_{p} \\
S(z)^{*}
\end{array}\right) \frac{y}{\rho_{z}(\zeta)}\right\rangle_{L_{2}^{p+q}(\mathbb{T})} \\
& =\left\langle\frac{I_{p}-S(\zeta) S(z)^{*}}{\rho_{z}(\zeta)} y, \frac{y}{\rho_{z}(\zeta)}\right\rangle_{L_{2}^{p}(\mathbb{T})}+\left\langle\frac{S(z)^{*}-S(\zeta)^{*}}{\rho_{z}(\zeta)} y, \frac{S(z)^{*} y}{\rho_{z}(\zeta)}\right\rangle_{L_{2}^{q}(\mathbb{T})} \\
& =y^{*} \frac{I_{p}-S(z) S(z)^{*}}{\rho_{z}(z)} y+\left\langle\frac{S(z)^{*} y}{\rho_{z}(\zeta)}, \frac{S(z)^{*} y}{\rho_{z}(\zeta)}\right\rangle_{L_{2}^{q}(\mathbb{T})}-\left\langle\frac{y}{\rho_{z}(\zeta)}, \frac{S(\zeta) S(z)^{*} y}{\rho_{z}(\zeta)}\right\rangle_{L_{2}^{p}(\mathbb{T})} \\
& =y^{*} \frac{I_{p}-S(z) S(z)^{*}}{\rho_{z}(z)} y
\end{aligned}
$$

and, in view of (1.6),

$$
\begin{aligned}
{\left[f_{1}, f_{2}\right]_{S} } & =\left\langle\left(\begin{array}{cc}
I_{p} & -S(\zeta) \\
-S(\zeta)^{*} & I_{q}
\end{array}\right) C G(\zeta)^{-1} x,\left(\begin{array}{c}
I_{p} \\
S(z)^{*}
\end{array}\right) \frac{y}{\rho_{z}(\zeta)}\right\rangle_{L_{2}^{p+q}(\mathbb{T})} \\
& =\left\langle\left(I_{p},-S(\zeta)\right) C G(\zeta)^{-1} x, \frac{y}{\rho_{z}(\zeta)}\right\rangle_{L_{2}^{p}(\mathbb{T})} \\
& =y^{*}\left(I_{p},-S(z)\right) C G(z)^{-1} x .
\end{aligned}
$$

Finally, in view of $(3.1)$,

$$
\left[f_{1}, f_{1}\right]_{S} \leq x^{*} P x .
$$

Substituting these three relations into (3.5) we get

$$
\left(\begin{array}{cc}
x^{*} P x & x^{*} G(z)^{-*} C^{*}\left(\begin{array}{c}
I_{p} \\
-S(z)^{*}
\end{array}\right) y \\
y^{*}\left(I_{p},-S(z)\right) C G(z)^{-1} x & y^{*} \frac{I_{p}-S(z) S(z)^{*}}{\rho_{z}(z)} y
\end{array}\right) \geq 0
$$

which is equivalent to (3.4) (since $x$ and $y$ are arbitrary).

The next step is to prove that in fact, the FMI (3.4) characterizes the set $\widehat{\mathcal{S}}(M, N, P, C)$ when (1.8) is in force. This will be done using the so called transformed matrix inequality which was introduced and applied to continuous interpolation problems by V. Katsnelson in [20] and [21]. See also [22], [23] and [25] for various applications of this idea. 
Lemma 3.4 Let the Lyapunov-Stein identity (1.8) be in force and let the FMI (3.4) hold for some $S \in \mathcal{S}^{p \times q}$. Then

1. The mvf

$$
W(z)=-H(z)^{-1} M^{*} P+H(z)^{-1} C_{1}^{*}\left(I_{p},-S(z)\right) C G(z)^{-1}
$$

is analytic in $\mathbb{D}$.

2. The mvf

$$
D(z)=H(z)^{-1} C^{*}\left(\begin{array}{c}
-S(z) \\
I_{q}
\end{array}\right)
$$

belongs $\mathbf{H}_{2}^{n \times q}$.

3. The transformed fundamental matrix inequality

$$
\left(\begin{array}{cc}
P & W(z)^{*} \\
W(z) & \frac{P+z W(z)+\bar{z} W(z)^{*}-D(z) D(z)^{*}}{\rho_{z}(z)}
\end{array}\right) \geq 0
$$

holds for every $z \in \mathbb{D}$.

4. If, in addition, (1.5) is valid, then $W \in \mathbf{H}_{2}^{n \times n}$.

5. If $\operatorname{rank} C_{1}=p$, then the inequalities (3.4) and (3.8) are equivalent.

Proof: Choose a point $z \in \mathbb{D}$ at which $G(z)$ and $H(z)$ are invertible and set

$$
E(z)=\left(\begin{array}{cc}
G(z)^{-*} & 0 \\
-H(z)^{-1} M^{*} G(z)^{-*} & H(z)^{-1} C_{1}^{*}
\end{array}\right) .
$$

Then, upon multiplying (3.4) by $E(z)$ on the left and by $E(z)^{*}$ on the right we get

$$
\left(\begin{array}{cc}
P & W(z)^{*} \\
W(z) & T(z)
\end{array}\right) \geq 0
$$

where $W(z)$ is the function defined by (3.6) and

$$
\begin{aligned}
T(z)=H(z)^{-1} & \left\{M^{*} P M-M^{*} G(z)^{-*} C^{*}\left(\begin{array}{c}
I_{p} \\
-S(z)^{*}
\end{array}\right) C_{1}\right. \\
& \left.-C_{1}^{*}\left(I_{p},-S(z)\right) C G(z)^{-1} M+C_{1}^{*} \frac{I_{p}-S(z) S(z)^{*}}{\rho_{z}(z)} C_{1}\right\} H(z)^{-*} .
\end{aligned}
$$

To simplify the last expression we use the resolventlike identity

$$
\rho_{z}(z) G(z)^{-1} M H(z)^{-*}=H(z)^{-*}-z G(z)^{-1}
$$

and the identity

$$
\begin{aligned}
\rho_{z}(z) H(z)^{-1} M^{*} P M H(z)^{-*}= & P-z H(z)^{-1} M^{*} P-\bar{z} P M H(z)^{-*} \\
& +H(z)^{-1}\left\{C_{1}^{*} C_{1}-C_{2}^{*} C_{2}\right\} H(z)^{-*}
\end{aligned}
$$


which follows easily from (1.8). Substituting (3.11) and (3.12) into (3.10) and using (3.6) and (3.7) we obtain

$$
\begin{aligned}
\rho_{z}(z) T(z)= & P-z H(z)^{-1} M^{*} P-\bar{z} P M H(z)^{-*}+H(z)^{-1}\left\{C_{1}^{*} C_{1}-C_{2}^{*} C_{2}\right\} H(z)^{-*} \\
& +H(z)^{-1} C_{1}^{*}\left\{I_{p}-S(z) S(z)^{*}\right\} C_{1} H(z)^{-*} \\
& +H(z)^{-1} C_{1}^{*}\left(I_{p},-S(z)\right) C\left(z G(z)^{-1}-H(z)^{-*}\right) \\
& +\left(\bar{z} G(z)^{-*}-H(z)^{-1}\right) C^{*}\left(\begin{array}{c}
I_{p} \\
-S(z)^{*}
\end{array}\right) C_{1} H(z)^{-*} \\
= & P+z W(z)+\bar{z} W(z)^{*}-D(z) D(z)^{*}
\end{aligned}
$$

and hence, by (3.9), the inequality (3.8) holds for every $z \in \mathbb{D}$ at which $G(z)$ and $H(z)$ are invertible. It follows from (3.8) that $T(z)$ is nonnegative inside $\mathbb{D}$ and therefore, (3.13) implies that

$$
P+z W(z)+\bar{z} W(z)^{*} \geq D(z) D(z)^{*} \geq 0 \quad(z \in \mathbb{D}) .
$$

Therefore the meromorphic function $z W(z)+\frac{1}{2} P$ has a nonnegative real part inside the unit disc and hence, is analytic there. Then we conclude from (3.13) that $T(z)$ is bounded on compact subsets of $\mathbb{D}$. Since both of the diagonal blocks $P$ and $T(z)$ in the nonnegative matrix (3.9) are bounded, the off-diagonal block $W(z)$ has to be bounded on compact subsets of $\mathbb{D}$ also and thus must be analytic in $\mathbb{D}$. Moreover, it follows from (3.14) that the meromorphic (by definition (3.7)) function $D(z)$ is bounded on compact subsets of $\mathbb{D}$ and is therefore analytic in $\mathbb{D}$. To show that $D$ belongs to $\mathbf{H}_{2}^{n \times q}$ we note that the expression on the left hand side of (3.14) is a nonnegative harmonic mvf which takes the value $P$ at the origin. Therefore,

$$
\frac{1}{2 \pi} \int_{0}^{2 \pi} D\left(r e^{i t}\right) D\left(r e^{i t}\right)^{*} d t \leq \frac{1}{2 \pi} \int_{0}^{2 \pi}\left(P+r e^{i t} W\left(r e^{i t}\right)+r e^{-i t} W\left(r e^{i t}\right)^{*}\right) d t=P .
$$

This implies the uniform estimate

$$
\sup _{0<r<1} \int_{0}^{2 \pi} D\left(r e^{i t}\right) D\left(r e^{i t}\right)^{*} d t \leq P
$$

which means that $D \in \mathbf{H}_{2}^{n \times q}$. Let us recall that the point $z \in \mathbb{D}$ was chosen so that $G(z)$ and $H(z)$ are invertible. Thus the inequality (3.8) is valid for all such points. Since all the entries in (3.8) are continuous in $\mathbb{D}$, the inequality extends by continuity to all $z \in \mathbb{D}$. This completes the proof of the first three assertions of the lemma.

Next, under assumption (1.5),

$$
\|W(\zeta)\| \leq \gamma \quad \text { for some } \gamma>0 \text { and a.e. } \zeta \in \mathbb{T},
$$

and therefore, $W \in \mathbf{H}_{2}^{n \times n}$.

To prove the last assertion of lemma we multiply the inequality $(3.8)$ by $\left(\begin{array}{cc}G(z)^{*} & 0 \\ M^{*} & H(z)\end{array}\right)$ from the left and by its adjoint from the right. Since

$$
\left(\begin{array}{cc}
G(z)^{*} & 0 \\
M^{*} & H(z)
\end{array}\right) E(z)=\left(\begin{array}{cc}
I_{n} & 0 \\
0 & C_{1}^{*}
\end{array}\right),
$$


the preceding analysis leads to the inequality

$$
\left(\begin{array}{cc}
G(z)^{*} P G(z) & C^{*}\left(\begin{array}{c}
I_{p} \\
-S(z)^{*}
\end{array}\right) C_{1} \\
C_{1}^{*}\left(I_{p},-S(z)\right) C & C_{1}^{*} \frac{I_{p}-S(z) S(z)^{*}}{\rho_{z}(z)} C_{1}
\end{array}\right) \geq 0 .
$$

But this is equivalent to (3.4) when $\operatorname{rank} C_{1}=p$.

Lemma 3.5 Let the Lyapunov-Stein identity (1.8) be in force and let $S$ be analytic in $\mathbb{D}$ and satisfy the FMI (3.4) at every point $z \in \mathbb{D}$. Then $S$ belongs to $\widehat{\mathcal{S}}(M, N, P, C)$.

Proof: By Lemma 3.4, the function $D$ given by (3.7) belongs to $\mathbf{H}_{2}^{n \times q}$. Therefore,

$$
\zeta D(\zeta)=\zeta H(\zeta)^{-1} C^{*}\left(\begin{array}{c}
-S(\zeta) \\
I_{q}
\end{array}\right) \text { belongs to } \zeta \mathbf{H}_{2}^{n \times q}
$$

Taking adjoints in the latter relation and taking advantage of the fact that

$$
G(\zeta)=\zeta H(\zeta)^{*} \quad(\text { for } \quad \zeta \in \mathbb{T})
$$

we get

$$
\left(-S(\zeta)^{*}, I_{q}\right) C G(\zeta)^{-1} \in\left(\mathbf{H}_{2}^{q \times n}\right)^{\perp} .
$$

We turn next to (3.1). By Lemma 3.4, the function $W$ given by (3.6) is analytic in $\mathbb{D}$. Hence, since $G(z)^{-1}$ and $H(z)^{-1}$ are rational mvf's and $S(z)$ is a Schur function, $W$ has nontangential limits $W(\zeta)$ for almost every point $\zeta \in \mathbb{T}$. Using $(3.15)$ we represent $W(\zeta)$ as

$$
W(\zeta)=-\bar{\zeta} G(\zeta)^{-*} M^{*} P+\bar{\zeta} G(\zeta)^{-*} C_{1}^{*}\left(I_{p},-S(\zeta)\right) C G(\zeta)^{-1}
$$

The identity

$$
P-G(\zeta)^{-*} M^{*} P-P M G(\zeta)^{-1}=-G(\zeta)^{-*} C J C G(\zeta)^{-1}
$$

is a consequence of (1.8) and implies together with (3.16), (1.9) and (1.2) that

$$
\begin{aligned}
\Phi(\zeta) & :=P+\zeta W(\zeta)+\bar{\zeta} W(\zeta)^{*} \\
& =G(\zeta)^{-*}\left(C_{1}^{*}\left(I_{p},-S(\zeta)\right) C+C^{*}\left(\begin{array}{c}
I_{p} \\
-S(\zeta)^{*}
\end{array}\right) C_{1}-C J C\right) G(\zeta)^{-1} \\
& =G(\zeta)^{-*}\left(C_{1}^{*} C_{1}-C_{1}^{*} S(\zeta) C_{2}-C_{2}^{*} S(\zeta) C_{1}+C_{2}^{*} C_{2}\right) G(\zeta)^{-1} \\
& =G(\zeta)^{-*} C^{*}\left(\begin{array}{cc}
I_{p} & -S(\zeta) \\
-S(\zeta)^{*} & I_{q}
\end{array}\right) C G(\zeta)^{-1}
\end{aligned}
$$

Let $\zeta_{1}=e^{i t_{1}}, \cdots, \zeta_{\ell}=e^{i t_{\ell}}$ be all the points on the unit circle at which $G(\zeta)$ is not invertible. Let $\Delta_{\varepsilon}$ be the set which consists of all points of $\mathbb{T}$ except for the $\ell \operatorname{arcs}\left[e^{i\left(t_{j}-\varepsilon\right)} ; e^{i\left(t_{j}+\varepsilon\right)}\right](j=$ $1, \ldots, \ell)$. It was mentioned in the proof of Lemma 3.4 that $\Phi$ is a nonnegative harmonic function which takes the value $P$ at the origin. By Fatou's lemma we get

$$
\begin{aligned}
\int_{\Delta_{\varepsilon}} \Phi\left(e^{i t}\right) d t & =\int_{\Delta_{\varepsilon}}\left(\lim _{r \rightarrow 1} \Phi\left(r e^{i t}\right)\right) d t \leq \liminf _{r \rightarrow 1} \int_{\Delta_{\varepsilon}} \Phi\left(r e^{i t}\right) d t \\
& \leq \liminf _{r \rightarrow 1} \int_{0}^{2 \pi} \Phi\left(r e^{i t}\right) d t=2 \pi \Phi(0)=2 \pi P .
\end{aligned}
$$


This yields the following estimate:

$$
\frac{1}{2 \pi} \int_{\Delta_{\varepsilon}} G\left(e^{i t}\right)^{-*} C^{*}\left(\begin{array}{cc}
I_{p} & -S\left(e^{i t}\right) \\
-S\left(e^{i t}\right)^{*} & I_{q}
\end{array}\right) C G\left(e^{i t}\right)^{-1} d t=\frac{1}{2 \pi} \int_{\Delta_{\varepsilon}} \Phi\left(e^{i t}\right) d t \leq P,
$$

which is uniform with respect to $\varepsilon$. Letting $\varepsilon \rightarrow 0$ we obtain

$$
\frac{1}{2 \pi} \int_{0}^{2 \pi} G\left(e^{i t}\right)^{-*} C^{*}\left(\begin{array}{cc}
I_{p} & -S\left(e^{i t}\right) \\
-S\left(e^{i t}\right)^{*} & I_{q}
\end{array}\right) C G\left(e^{i t}\right)^{-1} d t \leq P
$$

which is equivalent to $(3.1)$.

We now complete the proof of (1.6) with the help of the last inequality which can be written as

$$
\begin{aligned}
& \frac{1}{2 \pi} \int_{0}^{2 \pi} G\left(e^{i t}\right)^{-*} C^{*}\left(\begin{array}{c}
I_{p} \\
-S\left(e^{i t}\right)^{*}
\end{array}\right)\left(I_{p},-S\left(e^{i t}\right)\right) C G\left(e^{i t}\right)^{-1} d t \\
& \leq P-\frac{1}{2 \pi} \int_{0}^{2 \pi} G\left(e^{i t}\right)^{-*} C_{2}^{*}\left(I_{q}-S\left(e^{i t}\right)^{*} S\left(e^{i t}\right)\right) C_{2} G\left(e^{i t}\right)^{-1} d t .
\end{aligned}
$$

This implies in particular, that the function

$$
\left(I_{p},-S(\zeta)\right) C G(\zeta)^{-1} \quad \text { belongs to } L_{2}^{p \times n}(\mathbb{T}) .
$$

We recall that a mvf $B(z)$ which is analytic in $\mathbb{D}$ is said to belong to the Smirnov class $\mathcal{N}_{+}^{p \times q}$ if it can be represented as a ratio of a $\mathbf{H}_{\infty}^{p \times q}-$ function and a scalar $\mathbf{H}_{\infty}(\mathrm{D})$-function which is outer. The following maximum principle holds for functions of the Smirnov class: if $B \in \mathcal{N}_{+}^{p \times q}$ belongs to $L_{2}^{p \times q}(\mathbb{T})$, then $B$ also belongs to $\mathbf{H}_{2}^{p \times q}(\mathbb{D})$; for more information on matrix valued Smirnov classes see [6] and [24]. Let us show that the mvf

$$
B(z)=\left(I_{p},-S(z)\right) C G(z)^{-1}
$$

belongs to the Smirnov class $\mathcal{N}_{+}^{p \times n}$. Multiplying the matrix on the left hand side of (3.4) by $\left(\begin{array}{cc}G(z)^{-1} & 0 \\ 0 & I_{p}\end{array}\right)$ from the right, by its adjoint from the left and making use of the notation (3.19) we get the following inequality

$$
\left(\begin{array}{cc}
P & B(z)^{*} \\
B(z) & \frac{I_{p}-S(z) S(z)^{*}}{\rho_{z}(z)}
\end{array}\right) \geq 0
$$

which holds for all points $z \in \mathbb{D}$ at which $G(z)$ is invertible. The diagonal blocks $P$ and $\frac{I_{p}-S(z) S(z)^{*}}{\rho_{z}(z)}$ in the latter inequality are bounded on every compact subset of $\mathbb{D}$. Thus the offdiagonal block $B(z)$ (which is meromorphic by construction) is bounded on every compact subset of $\mathbb{D}$ and therefore, it is analytic in $\mathbb{D}$. Furthermore, the rational mvf $G^{-1}$ can be represented as

$$
G^{-1}(z)=\frac{G_{1}(z)}{g_{1}(z) g_{2}(z)}, \quad g_{1}(z) g_{2}(z)=\operatorname{det} G(z)
$$




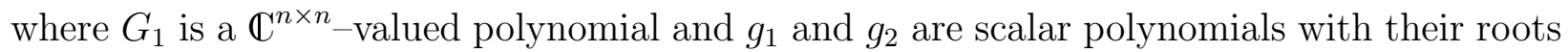
inside $\mathbb{D}$ and in $\mathbb{C} \backslash \mathbb{D}$, respectively. In particular, $g_{2}$ belongs to $\mathbf{H}_{\infty}(\mathbb{D})$ and is outer in $\mathbb{D}$. Substituting the latter representation for $G^{-1}$ into (3.19) we see that

$$
B(z)=\frac{\left(I_{p},-S(z)\right) C G_{1}(z)}{g_{1}(z)} \frac{1}{g_{2}(z)}
$$

and, since $B$ is analytic inside $\mathbb{D}$ it follows that all the zeroes of $g_{1}$ are compensated by the zeroes of the mvf $\left(I_{p},-S(z)\right) C G_{1}(z)$. Therefore, the mvf

$$
\frac{\left(I_{p},-S(z)\right) C G_{1}(z)}{g_{1}(z)} \text { belongs to } \mathbf{H}_{\infty}^{n \times n},
$$

and so by (3.20), $B$ belongs to the matrix Smirnov class $\mathcal{N}_{+}^{p \times n}$. By $(3.18), B \in L_{2}^{p \times n}(\mathbb{T})$ and thus by the maximum principle for Smirnov class functions,

$$
\left(I_{p},-S\left(e^{i t}\right)\right) C G\left(e^{i t}\right)^{-1} \quad \text { belongs to } \mathbf{H}_{2}^{p \times n}(\mathbb{T}) .
$$

This completes the proof that the function $S$ satisfies conditions (1.6) and (3.1) and therefore, belongs to $\widehat{\mathcal{S}}(M, N, P, C)$.

Remark 3.6 If in Lemma 3.5 it is also assumed that $G(\zeta)$ is invertible on the unit circle, then every solution $S$ of the FMI (3.4) belongs to the set $\mathcal{S}(M, N, P, C)$.

The stated conclusion is immediate from Lemmas 3.2 and 3.5. However, it is instructive to prove this fact independently of Lemma 3.5. This may be done by calculating the left hand side of (3.1) via formula (3.17). Because of (1.5), the function $W$ belongs to $\mathbf{H}_{2}^{n \times n}$ by Lemma 3.4. Therefore,

$$
\zeta W(\zeta) \in \zeta \mathbf{H}_{2}^{n \times n} \quad \text { and } \quad \bar{\zeta} W(\zeta)^{*} \in\left(\mathbf{H}_{2}^{n \times n}\right)^{\perp}
$$

Thus,

$$
\langle\zeta W(\zeta) y, x\rangle_{L_{2}^{n}(\mathbb{T})}=\left\langle\bar{\zeta} W(\zeta)^{*} y, x\right\rangle_{L_{2}^{n}(\mathbb{T})}=0 \quad\left(\forall x, y \in \mathbb{C}^{n}\right),
$$

and now it follows from (3.17), that for every choice of vectors $x$ and $y$ in $\mathbb{C}^{n}$,

$$
\langle\Phi(\zeta) y, x\rangle_{L_{2}^{n}(\mathbb{T})}=x^{*} P y=\left\langle G(\zeta)^{-*} C^{*}\left(\begin{array}{cc}
I_{p} & -S(\zeta) \\
-S(\zeta)^{*} & I_{q}
\end{array}\right) C G(\zeta)^{-1} y, x\right\rangle_{L_{2}^{n}(\mathbb{T})}
$$

which is equivalent to $(1.7)$.

As a consequence of the previous analysis we obtain the main result of this section:

Theorem 3.7 Let (1.17) be in force, let $P$ be a solution of the Lyapunov-Stein equation (1.8) and let $S$ be a $p \times q$ mvf which is analytic in $\mathbb{D}$. Then:

1. $S$ belongs to the set $\widehat{\mathcal{S}}(M, N, P, C)$ if and only if it is a solution of the FMI (3.4) at every point $z \in \mathbb{D}$. 
2. If (1.5) is in force, then $S$ is a solution of the $\operatorname{aBIP}(M, N, P, C)$ if and only if it satisfies the FMI (3.4) at every point $z \in \mathbb{D}$.

Proof: The first assertion of the theorem is immediate from Lemmas 3.3 and 3.5. Let now (1.5) be in force. By Lemma 3.2, relations (1.5) and (1.8) imply that $\widehat{\mathcal{S}}(M, N, P, C)$ coincides with $\mathcal{S}(M, N, P, C)$, which is, by Definition 3.1, the set of all solutions of the $\operatorname{aBIP}(M, N, P, C)$.

In Section 5 we will take advantage of the following simple but useful observation.

Corollary 3.8 Let $M_{1}$ and $N_{1}$ be $n \times n$ matrices such that the matrix pencil $G_{1}(z)=$ $M_{1}-z N_{1}$ is nondegenerate:

$$
\operatorname{det}\left(M_{1}-z N_{1}\right) \not \equiv 0
$$

and let

$$
P M=P M_{1} \quad \text { and } \quad P N=P N_{1} .
$$

Then $\widehat{\mathcal{S}}(M, N, P, C)=\widehat{\mathcal{S}}\left(M_{1}, N_{1}, P, C\right)$.

Proof: First we note that the conditions (3.22) imply that $P$ is a solution of the LyapunovStein equation (1.8) if and only if it is a solution of the equation

$$
M_{1}^{*} P M_{1}-N_{1}^{*} P N_{1}=C^{*} J C .
$$

Therefore, by Theorem 3.7, $S$ belongs to $\widehat{\mathcal{S}}(M, N, P, C)$ if and only if it satisfies the inequality (3.4), and it belongs to $\widehat{\mathcal{S}}\left(M_{1}, N_{1}, P, C\right)$ if and only if it satisfies the inequality

$$
\left(\begin{array}{cc}
G_{1}(z)^{*} P G_{1}(z) & C^{*}\left(\begin{array}{c}
I_{p} \\
-S(z)^{*}
\end{array}\right) \\
\left(I_{p},-S(z)\right) C & \frac{I_{p}-S(z) S(z)^{*}}{\rho_{z}(z)}
\end{array}\right) \geq 0 .
$$

However, in view of (3.22),

$$
G_{1}(z)^{*} P G_{1}(z)=\left(M_{1}-z N_{1}\right)^{*} P\left(M_{1}-z N_{1}\right)=(M-z N)^{*} P(M-z N)=G(z)^{*} P G(z)
$$

and the conditions (3.4) and (3.23) coincide.

\section{Some supplementary remarks}

In this section we explain briefly how to obtain a second Fundamental Matrix Inequality which characterizes the solutions of the aBIP. Let $\widetilde{S}$ be the dual function associated with $S$ by the rule

$$
\widetilde{S}(z)=S(\bar{z})^{*} \text {. }
$$

It is easily seen that $S$ belongs to the Schur class $\mathcal{S}^{p \times q}$ if and only if $\widetilde{S}$ belongs to $\mathcal{S}^{q \times p}$. The permutation matrix defined by the formula

$$
\Pi=\left(\begin{array}{cc}
0 & I_{q} \\
I_{p} & 0
\end{array}\right)
$$


will be used to interchange the block rows $C_{1}$ and $C_{2}$ in the decomposition (1.9) of $C$ :

$$
\Pi C=\Pi\left(\begin{array}{c}
C_{1} \\
C_{2}
\end{array}\right)=\left(\begin{array}{c}
C_{2} \\
C_{1}
\end{array}\right)
$$

Lemma 4.1 A function $S$ belongs to $\mathcal{S}(M, N, P, C)$ if and only if the dual function $\widetilde{S}$ belongs to $\mathcal{S}(N, M, P, \Pi C)$, i.e., if and only if

$$
\left(\begin{array}{cc}
I_{q} & -\widetilde{S}(\zeta) \\
-\widetilde{S}(\zeta)^{*} & I_{p}
\end{array}\right) \Pi C H(\bar{\zeta})^{-*} \in\left(\begin{array}{c}
\mathbf{H}_{2}^{q \times n} \\
\left(\mathbf{H}_{2}^{p \times n}\right)^{\perp}
\end{array}\right)
$$

and for every choice of $x, y \in \mathbb{C}^{n}$,

$$
\left\langle\left(\begin{array}{cc}
I_{q} & -\widetilde{S}(\zeta) \\
-\widetilde{S}(\zeta)^{*} & I_{p}
\end{array}\right) \Pi C H(\bar{\zeta})^{-*} y, \Pi C H(\bar{\zeta})^{-*} x\right\rangle_{L_{2}^{p+q}(\mathbb{T})}=x^{*} P y
$$

Proof: Using (4.1), (4.2) and (3.15), we get

$$
\left(\begin{array}{cc}
I_{p} & -S(\zeta) \\
-S(\zeta)^{*} & I_{q}
\end{array}\right) C G(\zeta)^{-1}=\bar{\zeta} \Pi^{*}\left(\begin{array}{cc}
I_{q} & -\widetilde{S}(\bar{\zeta}) \\
-\widetilde{S}(\bar{\zeta})^{*} & I_{p}
\end{array}\right) \Pi C H(\zeta)^{-*}
$$

Therefore, condition (1.6) is equivalent to

$$
\bar{\zeta}\left(\begin{array}{cc}
I_{q} & -\widetilde{S}(\bar{\zeta}) \\
-\widetilde{S}(\bar{\zeta})^{*} & I_{p}
\end{array}\right) \Pi C H(\zeta)^{-*} \in\left(\begin{array}{c}
\left(\mathbf{H}_{2}^{q \times n}\right)^{\perp} \\
\mathbf{H}_{2}^{p \times n}
\end{array}\right) .
$$

It is easily seen that a function $f(\zeta)$ belongs to $\mathbf{H}_{2}$ (respectively $\left(\mathbf{H}_{2}\right)^{\perp}$ ) if and only if the function $\bar{\zeta} f(\bar{\zeta})$ belongs to $\left(\mathbf{H}_{2}\right)^{\perp}$ (respectively $\mathbf{H}_{2}$ ) and hence that (4.6) is equivalent to (4.3). Next, upon substituting (3.15) and (4.5) into (1.7) and taking into account that $\Pi$ is unitary, we conclude that the expressions on the left hand sides of (1.7) and (4.4) coincide and therefore that these two conditions are equivalent.

Remark 4.2 It follows immediately from the proof of the last lemma that a function $S$ belongs to $\widehat{\mathcal{S}}(M, N, P, C)$ if and only if the dual function $\widetilde{S}$ belongs to $\widehat{\mathcal{S}}(N, M, P, \Pi C)$. Moreover, by Lemmas 3.3 and 3.5, $\widetilde{S}$ belongs to $\widehat{\mathcal{S}}(N, M, P, \Pi C)$ if and only if the inequality (3.4) holds, with $M$ and $N$ interchanged and with $C$ and $S$ replaced by $\Pi C$ and $\widetilde{S}$, respectively. That is if and only if

$$
\left(\begin{array}{cc}
H(\bar{z}) P H(\bar{z})^{*} & C^{*} \Pi^{*}\left(\begin{array}{c}
I_{q}, \\
-\widetilde{S}(z)^{*}
\end{array}\right) \\
\left(I_{q},-\widetilde{S}(z)\right) \Pi C & \frac{I_{q}-\widetilde{S}(z) \widetilde{S}(z)^{*}}{\rho_{z}(z)}
\end{array}\right) \geq 0 \quad(\forall z \in \mathbb{D}) .
$$

Upon replacing $z$ by $\bar{z}$ and expressing $\widetilde{S}$ in terms of $S$ we obtain we obtain the dual fundamental matrix inequality, which also characterizes the set $\widehat{\mathcal{S}}(M, N, P, C)$ : 
Theorem 4.3 Let $P \geq 0$ be a solution of the Lyapunov-Stein equation (1.8). Then a $p \times q$ mvf $S$ which is analytic in $\mathbb{D}$ belongs to $\widehat{\mathcal{S}}(M, N, P, C)$ if and only if the inequality

$$
\left(\begin{array}{cc}
H(z) P H(z)^{*} & C^{*}\left(\begin{array}{c}
-S(z) \\
I_{q}
\end{array}\right) \\
\left(-S(z)^{*}, I_{q}\right) C & \frac{I_{q}-S(z)^{*} S(z)}{\rho_{z}(z)}
\end{array}\right) \geq 0
$$

holds for every $z \in \mathbb{D}$.

The same strategy can be used to obtain the dual analogue of Lemma 3.4. It suffices to interchange $M$ and $N, C_{1}$ and $C_{2}$ and to replace $S$ by the dual function $\widetilde{S}$ in formulas (3.6) $-(3.8)$ and then to make use of (4.1). The details are left to the reader.

Lemma 4.4 Let the Lyapunov-Stein identity (1.8) be in force and let the fundamental matrix inequality (4.7) hold for some $S \in \mathcal{S}^{p \times q}$. Then

1. The mvf

$$
\widetilde{W}(z)=P N G(z)^{-1}+H(z)^{-1} C^{*}\left(\begin{array}{c}
-S(z) \\
I_{q}
\end{array}\right) C_{2} G(z)^{-1}
$$

is analytic in $\mathbb{D}$ whereas the mvf

$$
\widetilde{D}(z)=\left(I_{p},-S(z)\right) C G(z)^{-1}
$$

belongs $\mathbf{H}_{2}^{p \times n}$, and the transformed fundamental matrix inequality

$$
\left(\begin{array}{cc}
P & \widetilde{W}(z) \\
\widetilde{W}(z)^{*} & \frac{P+z \widetilde{W}(z)+\bar{z} \widetilde{W}(z)^{*}-\widetilde{D}(z)^{*} \widetilde{D}(z)}{\rho_{z}(z)}
\end{array}\right) \geq 0
$$

holds for every $z \in \mathbb{D}$.

2. If in addition, (1.5) is valid, then $\widetilde{W} \in \mathbf{H}_{2}^{n \times n}$.

3. If $\operatorname{rank} C_{2}=q$, then the inequalities (4.7) and (4.8) are equivalent.

In the last two sections we obtained two characterizations of the set $\widehat{\mathcal{S}}(M, N, P, C)$ : as the set of all solutions of the FMI (3.4) and as the set of all solutions of the dual FMI (4.7). As a corollary we get the following result.

Lemma 4.5 Let the Lyapunov-Stein identity (1.8) be in force. Then a $p \times q$ mvf $S$ which is analytic in $\mathbb{D}$ satisfies (3.4) for every point $z \in \mathbb{D}$ if and only if it satisfies (4.7) for every point $z \in \mathbb{D}$.

A direct proof of this fact for the more general Abstract Interpolation Problem can be found in [23]. Although the inequalities (3.4) and (4.7) are equivalent, their simultaneous use seems to be convenient and fruitful; for applications to concrete interpolation problems see [12] and [26]. 


\section{Some nonrestrictive assumptions}

The objective of this section is to formulate a new aBIP which is equivalent to the original problem but with extra invariance properties. In what follows $\mathbf{U} \mathbf{C}^{n \times n}$ denotes the set of upper triangular $n \times n$ matrices and for each square matrix $R, D_{R}$ denotes the diagonal matrix which has the same main diagonal as $R$.

Lemma 5.1 Let $\mathbf{T} \in \mathbf{U C}^{n \times n}$ be a matrix with $D_{\mathbf{T}}=I_{n}$ and let $\Phi \in \mathbf{U C}^{n \times n}$. Then $\mathbf{T} \Phi \mathbf{T}^{-1}$ and $\mathbf{T}^{-1} \Phi \mathbf{T}$ belong to $\mathbf{U} \mathbf{C}^{n \times n}$ and have the same main diagonals as $\Phi$.

Proof: Clearly,

$$
\Phi=D_{\Phi}+\widetilde{\Phi}, \quad T=I_{n}+\widetilde{T} \quad \text { and } \quad T^{-1}=I_{n}+\widehat{T},
$$

where $\widetilde{\Phi}, \widetilde{T}$ and $\widehat{T}$ are strictly upper triangular matrices. Thus

$$
\mathbf{T} \Phi \mathbf{T}^{-1}=D_{\Phi}+\Psi \quad \text { and } \quad \mathbf{T}^{-1} \Phi \mathbf{T}=D_{\Phi}+\widetilde{\Psi}
$$

where $\Psi$ and $\widetilde{\Psi}$ are the sums of matrix products each of which has at least one strictly upper triangular factor and is therefore, strictly upper triangular. Therefore the main diagonals of $\mathbf{T} \Phi \mathbf{T}^{-1}$ and $\mathbf{T}^{-1} \Phi \mathbf{T}$ coincide with the main diagonal of $\Phi$.

Lemma 5.2 Let $P \in \mathbb{C}^{n \times n}$ be positive semidefinite with rank $P=r \leq n$. Then there exists a matrix $Q \in \mathbb{C}^{n \times r}$ and linear transformations $\widehat{\tau}: R \rightarrow \widehat{R}$ of $\mathbf{U C}^{n \times n}$ into itself and $\tau_{\diamond}: R \rightarrow R_{\diamond}$ of $\mathbf{U C}^{n \times n}$ into $\mathbf{U C}^{r \times r}$ such that:

(1) $\quad Q^{*} P Q>0$.

(2) $\quad D_{R}=D_{\widehat{R}}$.

(3) $\quad P R=P \widehat{R}$.

(4) $\widehat{R} Q=Q R_{\diamond}$.

(5) $\quad \operatorname{spec} R_{\diamond} \subseteq \operatorname{spec} R$.

Proof: We first show that there exists a matrix $\mathbf{T} \in \mathbf{U C}^{n \times n}$ with $D_{\mathbf{T}}=I_{n}$ such that

$$
P=\mathbf{T}^{*} P_{d} \mathbf{T}, \quad \text { where } \quad P_{d}:=\operatorname{diag}\left(k_{1}, k_{2}, \cdots, k_{n}\right) \geq 0 .
$$

The matrix $\mathbf{T}$ is constructed by successive application of Schur complements: setting

$$
P^{(1)}:=P, \quad k_{1}=P_{11}^{(1)}=(1,0, \ldots, 0) P^{(1)}(1,0, \ldots, 0)^{*}
$$

and using the partition

$$
P^{(\ell)}=\left(\begin{array}{ll}
k_{\ell} & F_{\ell} \\
F_{\ell}^{*} & \widehat{P}_{\ell}
\end{array}\right):\left(\begin{array}{l}
\mathbb{C} \\
\mathbb{C}^{n-\ell}
\end{array}\right) \rightarrow\left(\begin{array}{l}
\mathbb{C} \\
\mathbb{C}^{n-\ell}
\end{array}\right),
$$

let

$$
P^{(\ell+1)}=\left\{\begin{array}{lll}
\widehat{P}_{\ell}-F_{\ell}^{*} k_{\ell}^{-1} F_{\ell} & \text { if } & k_{\ell}>0 \\
\widehat{P}_{\ell} & \text { if } & k_{\ell}=0
\end{array}\right.
$$


and

$$
T_{\ell}=\left\{\begin{array}{ccc}
\left(\begin{array}{ccc}
I_{\ell-1} & 0 & 0 \\
0 & 1 & k_{\ell}^{-1} F_{\ell} \\
0 & 0 & I_{n-\ell}
\end{array}\right) & \text { if } \quad k_{\ell}>0, \\
& I_{n} & \text { if } \quad k_{\ell}=0,
\end{array}\right.
$$

for $\ell=1, \ldots, n$. The matrix $\mathbf{T}:=T_{n} T_{n-1} \cdots T_{1}$ clearly does the trick by standard manipulations with Schur complements.

Since $\operatorname{det} \mathbf{T} \neq 0$, it follows from (5.5) that the diagonal matrix $P_{d}$ has the same rank as $P$. Thus, there are exactly $r$ strictly positive elements in the set $\mathcal{K}=\left\{k_{1}, \ldots, k_{n}\right\}$. Let $\mathcal{N}$ denote the set of indices of these strictly positive elements:

$$
\begin{aligned}
& k_{\ell}>0 \quad \text { for } \quad \ell \in\left\{i_{1}, i_{2}, \ldots, i_{r}\right\}=: \mathcal{N}, \quad i_{1}<i_{2}<\ldots<i_{r} \text {; } \\
& k_{\ell}=0 \quad \text { for } \quad \ell \in\{1, \ldots, n\} \backslash \mathcal{N}
\end{aligned}
$$

and let

$$
E_{\mathcal{N}}=\left(\mathbf{e}_{i_{1}}, \mathbf{e}_{i_{2}}, \ldots, \mathbf{e}_{i_{r}}\right),
$$

where $\mathbf{e}_{j}$ denotes the $j$-th column of the matrix $I_{n}$. Then $E_{\mathcal{N}} \in \mathbb{C}^{n \times r}$ is isometric and by the definition (5.6) of the set $\mathcal{N}$, it follows from (5.5) that

$$
E_{\mathcal{N}}^{*} P_{d} E_{\mathcal{N}}=\operatorname{diag}\left\{k_{i_{1}}, k_{i_{2}}, \ldots, k_{i_{r}}\right\}>0 \text { and } P_{d} E_{\mathcal{N}} E_{\mathcal{N}}^{*}=P_{d}
$$

Next, let

$$
Q=\mathbf{T}^{-1} E_{\mathcal{N}} \in \mathbb{C}^{n \times r}
$$

and let us define the mappings $\widehat{\tau}: \quad \mathbf{U C}^{n \times n} \rightarrow \mathbf{U C}^{n \times n}$ and $\tau_{\diamond}: \quad \mathbf{U C}^{n \times n} \rightarrow \mathbf{U C}^{r \times r}$ by the rules

$$
\widehat{\tau} R=\widehat{R}:=\mathbf{T}^{-1}\left\{E_{\mathcal{N}} E_{\mathcal{N}}^{*} \mathbf{T} R \mathbf{T}^{-1}+\left(I_{n}-E_{\mathcal{N}} E_{\mathcal{N}}^{*}\right) D_{R}\right\} \mathbf{T}
$$

and

$$
\tau_{\diamond} R=R_{\diamond}:=E_{\mathcal{N}}^{*} \mathbf{T} R \mathbf{T}^{-1} E_{\mathcal{N}},
$$

respectively. These mappings are obviously linear and the matrices $\widehat{R}$ and $R_{\diamond}$ are upper triangular by Lemma 5.1. Now we show that the requirements (5.1)-(5.4) of the lemma are satisfied for this choice of $Q, \widehat{R}$ and $R_{\diamond}$.

Relations (5.1) and (5.3) follow from (5.5) and (5.8)-(5.10):

$$
Q^{*} P Q=E_{\mathcal{N}}^{*} \mathbf{T}^{-*} P \mathbf{T}^{-1} E_{\mathcal{N}}=E_{\mathcal{N}}^{*} P_{d} E_{\mathcal{N}}>0
$$

and

$$
\begin{aligned}
P \widehat{R} & =\mathbf{T}^{*} P_{d}\left\{E_{\mathcal{N}} E_{\mathcal{N}}^{*} \mathbf{T} R \mathbf{T}^{-1}+\left(I_{n}-E_{\mathcal{N}} E_{\mathcal{N}}^{*}\right) D_{R}\right\} \mathbf{T} \\
& =\mathbf{T}^{*} P_{d} \mathbf{T} R=P R .
\end{aligned}
$$

Next, by Lemma 5.1 and the definition of $D_{R}$, all the diagonal entries of the matrix $\mathbf{T} R \mathbf{T}^{-1}-$ $D_{R}$ are zero. Since the matrix $E_{\mathcal{N}} E_{\mathcal{N}}^{*}$ is diagonal, all the diagonal elements in the matrix 
$E_{\mathcal{N}} E_{\mathcal{N}}^{*}\left(\mathbf{T} R \mathbf{T}^{-1}-D_{R}\right)$ are zero also. Therefore, upon representing the expression inside the brackets in (5.10) as

$$
D_{R}+E_{\mathcal{N}} E_{\mathcal{N}}^{*}\left(\mathbf{T} R \mathbf{T}^{-1}-D_{R}\right)
$$

and applying Lemma 5.1 again, we get (5.2).

To prove (5.4), we express $D_{R}$ as $D_{R}=\operatorname{diag}\left\{R_{j j}\right\}_{j=1}^{n}$ and note that

$$
D_{R} E_{\mathcal{N}}=E_{\mathcal{N}} \operatorname{diag}\left\{R_{i_{k} i_{k}}\right\}_{k=1}^{r} .
$$

Therefore, since $E_{\mathcal{N}}$ is an isometric matrix, $\left(I_{n}-E_{\mathcal{N}} E_{\mathcal{N}}^{*}\right) D_{R} E_{\mathcal{N}}=0$ and hence

$$
\begin{aligned}
\widehat{R} Q & =\mathbf{T}^{-1}\left\{E_{\mathcal{N}} E_{\mathcal{N}}^{*} \mathbf{T} R \mathbf{T}^{-1}+\left(I_{n}-E_{\mathcal{N}} E_{\mathcal{N}}^{*}\right) D_{R}\right\} E_{\mathcal{N}} \\
& =\mathbf{T}^{-1} E_{\mathcal{N}} E_{\mathcal{N}}^{*} \mathbf{T} R \mathbf{T}^{-1} E_{\mathcal{N}}=Q R_{\diamond} .
\end{aligned}
$$

The last assertion of lemma follows easily from (5.11) by Lemma 5.1.

Combining Corollary 3.8 and Lemma 5.1 we deduce the main result of this section. We recall that $\widehat{\mathcal{S}}(M, N, P, C)$ is the set given in Definition 3.1.

Theorem 5.3 Let $M, N \in \mathbb{C}^{n \times n}$ be upper triangular matrices satisfying (1.17) and let $P$ be a positive semidefinite solution of the Lyapunov-Stein equation (1.8) with $\operatorname{rank} P=r \leq n$. Then there exists a matrix $Q \in \mathbb{C}^{n \times r}$ satisfying (5.1) and upper triangular matrices $M_{1}, N_{1} \in$ $\mathbf{U C}^{n \times n}$ and $M_{\diamond}, N_{\diamond} \in \mathbf{U C}^{r \times r}$ such that:

(1) $\hat{\mathcal{S}}(M, N, P, C)=\widehat{\mathcal{S}}\left(M_{1}, N_{1}, P, C\right)$.

(2) $\quad M_{1} Q=Q M_{\diamond} \quad$ and $\quad N_{1} Q=Q N_{\diamond}$

(3) $\quad \operatorname{spec} M_{\diamond} \subseteq \operatorname{spec} M=\operatorname{spec} M_{1} \quad$ and $\quad \operatorname{spec} N_{\diamond} \subseteq \operatorname{spec} N=\operatorname{spec} N_{1}$.

Proof: Let the matrix $Q$ and mappings $\widehat{\tau}$ and $\tau_{\diamond}$ be as in Lemma 5.2 and let

$$
M_{1}=\widehat{\tau} M, \quad N_{1}=\widehat{\tau} N, \quad M_{\diamond}=\tau_{\diamond} M, \quad N_{\diamond}=\tau_{\diamond} N .
$$

Then, relations (5.1), (5.12), (5.13) and (3.22) hold by Lemma 5.1. Since the functions $M-z N$ and $M_{1}-z N_{1}$ are upper triangular and have the same main diagonal for each $z$, the condition (3.21) is an immediate consequence of (1.17). Applying Corollary 3.8, we conclude that $\widehat{\mathcal{S}}(M, N, P, C)=\widehat{\mathcal{S}}\left(M_{1}, N_{1}, P, C\right)$.

Remark 5.4 Let $P \in \mathbb{C}^{n \times n}$ be a positive semidefinite solution of the Lyapunov-Stein equation (1.8) and let rank $P=r \leq n$. In view of Theorem 5.3, it can be assumed without loss of generality that $M$ and $N$ satisfy the invariance conditions

$$
M Q=Q M_{\diamond} \quad \text { and } \quad N Q=Q N_{\diamond}
$$

for some matrices $M_{\diamond}, N_{\diamond} \in \mathbb{C}^{r \times r}$ satisfying the spectral conditions (5.13) and a matrix $Q \in \mathbb{C}^{n \times r}$ satisfying the condition (5.1). 
Remark 5.5 It follows from (5.14) that $\operatorname{det}\left(M_{\diamond}-z N_{\diamond}\right) \neq 0$ whenever $\operatorname{det}(M-z N) \neq 0$.

Proof: In view of (5.14),

$$
(M-z N) Q=Q\left(M_{\diamond}-z N_{\diamond}\right),
$$

and hence, if $\operatorname{det}(M-z N) \neq 0$, then

$$
\operatorname{rank} Q\left(M_{\diamond}-z N_{\diamond}\right)=\operatorname{rank}(M-z N) Q=\operatorname{rank} Q=r .
$$

Therefore, $\operatorname{rank}\left(M_{\diamond}-z N_{\diamond}\right)=r$, which means that $M_{\diamond}-z N_{\diamond}$ is invertible.

The next example shows that in general one cannot achieve the invariance exhibited in (5.14) without modifying $M$ and $N$.

Example 5.6 Let $p=2, q=1$, and let

$$
P=\left(\begin{array}{ll}
0 & 0 \\
0 & 1
\end{array}\right), \quad M=\left(\begin{array}{ll}
1 & 1 \\
0 & 1
\end{array}\right), \quad N=\left(\begin{array}{ll}
0 & 1 \\
0 & 0
\end{array}\right), \quad C_{1}=\left(\begin{array}{ll}
1 & 0 \\
0 & 1
\end{array}\right), \quad C_{2}=(1,0) .
$$

Then (1.8) holds and $\operatorname{rank} P=1$. Let us assume that there exist matrices

$$
Q=\left(\begin{array}{c}
\alpha \\
\beta
\end{array}\right) \in \mathbb{C}^{2 \times 1} \text { and } D \in \mathbb{C}^{1 \times 1}
$$

such that

$$
Q^{*} P Q=|\beta|^{2}>0 \text { and }\left(\begin{array}{c}
\beta \\
0
\end{array}\right)=N Q=Q D=\left(\begin{array}{c}
\alpha D \\
\beta D
\end{array}\right) .
$$

The second relation in (5.15) implies that $\beta D=0$ and $\beta=\alpha D$ and therefore $\beta=0$, which contradicts the first relation in (5.15).

To conclude the section, we wish to clarify the geometrical meaning of conditions (5.1) and (5.14). Since $Q \in \mathbb{C}^{n \times r}$ and $\operatorname{rank} P=r$, it follows from the first of these two conditions that the space $\mathbb{C}^{n}$ can be decomposed as

$$
\mathbb{C}^{n}=\operatorname{Ker} P \dot{+} \mathcal{Q}
$$

where the sum is direct and $\mathcal{Q}$ is the subspace given by

$$
\mathcal{Q}=\operatorname{Ran} Q:=\left\{Q x, \quad x \in \mathbb{C}^{r}\right\} \subset \mathbb{C}^{n} .
$$

Indeed, in view of (5.1), $Q x \neq 0$ for any nonzero $x \in \mathbb{C}^{r}$ and therefore, $\operatorname{rank} Q=r$. Thus

$$
\operatorname{dim} \mathcal{Q}=\operatorname{rank} Q=\operatorname{rank} P=r .
$$

On the other hand, if $y \in \operatorname{Ker} P \cap \mathcal{Q}$, then

$$
P y=0 \quad \text { and } \quad y=Q x \quad \text { for some } \quad x \in \mathbb{C}^{r} .
$$


Therefore,

$$
Q^{*} P y=Q^{*} P Q x=0,
$$

which, on account of (5.1), implies that $x=0$ and hence, that $y=0$. Thus, Ker $P \cap \mathcal{Q}=\{0\}$ and therefore,

$$
\operatorname{dim}(\operatorname{Ker} P+\mathcal{Q})=\operatorname{dim} \mathcal{Q}+\operatorname{dim} \operatorname{Ker} P=n,
$$

which implies (5.16). Next, the relations (5.14) mean that $\mathcal{Q}$ is an invariant subspace of $M$ and $N$, i.e.,

$$
M \mathcal{Q} \subseteq \mathcal{Q} \text { and } N \mathcal{Q} \subseteq \mathcal{Q}
$$

A decomposition of the form (5.16) was used by V. Dubovoj in [11] to study the degenerate matrix Schur problem. This corresponds to the choice $M=I_{n}$ and $N$ equal to the block shift matrix. It turns out that in this case the modification introduced in Theorem 5.3 is not needed.

The invariance properties of the subspace $\mathcal{Q}$ will enable us to construct a linear fractional transformation describing all the solutions of the degenerate $\operatorname{aBIP}(M, N, P, C)$. This will be done in the next section.

\section{The matrix valued function $\Delta_{\omega}(z)$}

In this section we consider the $n \times n$ matrix valued function

$$
\Delta_{\omega}(z)=G(\omega)^{*} P G(z)+\rho_{\omega}(z) C_{2}^{*} C_{2} .
$$

which was introduced in [15] and used extensively in [15] and [16] (in a more general setting) and which will play an important role in this paper too. We begin with a list of formulas which are taken from [15] and [16]. They can be verified by straightforward computation, especially if they are tackled in the order in which they are stated.

Lemma 6.1 If $P \geq 0$ is a solution of the Lyapunov-Stein equation (1.10), then the following formulas are valid for every choice of $z$ and $\omega$ in $\mathbb{C}$ :

(1) $\Delta_{\omega}(z)=H(z) P H(\omega)^{*}+\rho_{\omega}(z) C_{1}^{*} C_{1}$,

(2) $\Delta_{\omega}(z)=\Delta_{z}(\omega)^{*}$,

(3) $\rho_{\omega}(z) G(z)^{*} P G(\omega)+\rho_{\omega}(z)^{*} H(z) P H(\omega)^{*}$

$$
=\rho_{z}(z) G(\omega)^{*} P G(\omega)+\rho_{\omega}(\omega) H(z) P H(z)^{*},
$$

(4) $\rho_{\omega}(z)^{*} \Delta_{\omega}(z)+\rho_{\omega}(z) \Delta_{\omega}(z)^{*}=\rho_{z}(z) G(\omega)^{*} P G(\omega)+\rho_{\omega}(\omega) H(z) P H(z)^{*}$

$$
+\left|\rho_{\omega}(z)\right|^{2}\left(C_{1}^{*} C_{1}+C_{2}^{*} C_{2}\right) \text {. }
$$


There are two cases of interest in this paper: the case when $\omega \in \mathbb{D}$ and the case when $\omega \in \mathbb{T}$ and $G(\omega)$ is invertible. For both the cases the subspace

$$
\mathcal{K}=\operatorname{Ker}\left(\begin{array}{c}
P M \\
P N \\
C
\end{array}\right)
$$

of $\mathbb{C}^{n}$ plays a significant role. First we note the inclusion

$$
\operatorname{Ker} \Delta_{\omega}(z) \cap \operatorname{Ker} \Delta_{\omega}(z)^{*} \supseteq \mathcal{K} \quad \text { for all } z, \omega \in \mathbb{C},
$$

which is selfevident by definitions (6.1) and (6.5). We are interested in the cases when equality prevails in (6.6).

Lemma 6.2 Let $P \geq 0$ be a solution of the Lyapunov-Stein equation (1.10) and let $\omega \in \mathbb{D}$. Then

$$
\operatorname{Ker} \Delta_{\omega}(z)=\operatorname{Ker} \Delta_{\omega}(z)^{*}=\mathcal{K}
$$

for every point $z \in \mathbb{D}$ and for every point $z \in \mathbb{T}$ at which $G(z)$ is invertible.

Proof: Fix $\omega \in \mathbb{D}$ and let $u \in \operatorname{Ker} \Delta_{\omega}(z) \cup \operatorname{Ker} \Delta_{\omega}(z)^{*}$. Then

$$
u^{*}\left\{\rho_{\omega}(z)^{*} \Delta_{\omega}(z)+\rho_{\omega}(z) \Delta_{\omega}(z)^{*}\right\} u=0
$$

and hence, as $\rho_{\omega}(\omega)>0$ for $\omega \in \mathbb{D}$ it follows from (6.4) that

$$
P H(z)^{*} u=0, \quad C_{1} u=0, \quad C_{2} u=0
$$

and

$$
\rho_{z}(z) P G(\omega) u=0 .
$$

Now, if $z \in \mathbb{D}$, then $\rho_{z}(z)>0$ and so formula (6.10) implies that $P G(\omega) u=0$. Therefore,

$$
(1-\bar{z} \omega) P N u=\bar{z} P G(\omega) u-P H(z)^{*} u=0,
$$

which implies that $P N u=0$ and hence by (6.9) that $P M u=0$ also. This proves the inclusion

$$
\operatorname{Ker} \Delta_{\omega}(z) \cup \operatorname{Ker} \Delta_{\omega}(z)^{*} \subseteq \mathcal{K} \quad \text { if } \quad z \in \mathbb{D}
$$

and so, in view of (6.6), the equalities (6.7) for $z \in \mathbb{D}$.

Next, if $z \in \mathbb{T}$, then there is no information in formula (6.10). However, if $G(z)$ is invertible (or equivalently, if $H(z)$ is invertible; see (2.5)), then (6.1), (6.2) and (6.9) imply that

$$
\text { 1) } P H(\omega)^{*} u=0 \text { if } u \in \operatorname{Ker} \Delta_{\omega}(z) \text { and 2) } P G(\omega) u=0 \quad \text { if } u \in \operatorname{Ker} \Delta_{\omega}(z)^{*} \text {. }
$$

In the first case we have

$$
(\bar{z}-\bar{\omega}) P N u=\bar{\omega} P H(z)^{*} u-\bar{z} P H(\omega)^{*} u=0,
$$

which implies that $P N u=0$ and hence by the first equality in (6.9) that $P M u=0$ also. In the second case the same conclusions follow from (6.11). Thus, $u \in \mathcal{K}$, which on account of (6.6), completes the proof.

The situation in the case when $\omega \in \mathbb{T}$ is almost the same. 
Lemma 6.3 Let $P \geq 0$ be a solution of the Lyapunov-Stein equation (1.10), let $\omega \in \mathbb{T}$ and let $G(\omega)$ be invertible. Then the qualities (6.7) hold for every point $z \in \mathbb{D}$ and for every point $z \in \mathbb{T} \backslash\{\omega\}$ at which $G(z)$ is invertible.

Proof: The assertion of the lemma for $z \in \mathbb{D}$ follows by property (6.3) from Lemma 6.2 after interchanging $z$ and $\omega$ in (6.7).

Next, if $z$ is any point on $\mathbb{T}$ different from $\omega$ and $u \in \operatorname{Ker} \Delta_{\omega}(z)$ or $u \in \operatorname{Ker} \Delta_{\omega}(z)^{*}$, then $\rho_{\omega}(z) \neq 0$ and (6.8) implies only that $C_{1} u=0$ and $C_{2} u=0$. However, since $G$ and $H$ are invertible at $z$ and at $\omega$, it follows from (6.1) and (6.2) that

$$
P H(\omega)^{*} u=P G(z) u=P G(\omega) u=P H(z)^{*} u=0 .
$$

The proof is now readily completed as before.

The case when $z=\omega \in \mathbb{T}$, is exceptional: then $\Delta_{\omega}(\omega)=G(\omega)^{*} P G(\omega)$ and therefore, the kernel of $\Delta_{\omega}(\omega)$ can be strictly included in $\mathcal{K}$.

Lemma 6.4 Let $P$ be a nonnegative solution of the Lyapunov-Stein equation (1.8), let $\mu \in$ $\mathbb{T}$ be a point at which $G(\mu)$ is invertible and let

$$
\mathcal{R}:=\operatorname{Ran} C G(\mu)^{-1} \mathbf{P}_{\operatorname{Ker} P}=\left\{C G(\mu)^{-1} g: \quad g \in \operatorname{Ker} P\right\} \subseteq \mathbb{C}^{p+q},
$$

where $\mathbf{P}_{\text {KerP }}$ denotes the orthogonal projection of $\mathbb{C}^{n}$ onto $\operatorname{Ker} P$. Then

$$
\operatorname{rank} \Delta_{\omega}(\omega)= \begin{cases}\operatorname{rank} P+\operatorname{dim} \mathcal{R} & \text { if } \omega \in \mathbb{D} \\ \operatorname{rank} P^{\frac{1}{2}} G(\omega) & \text { if } \omega \in \mathbb{T} .\end{cases}
$$

Proof: The case of $|\omega|=1$ is selfevident, since according to $(6.1), \Delta_{\omega}(\omega)=G(\omega)^{*} P G(\omega)$. Let $\omega \in \mathbb{D}$. Then by Lemma 6.2 ,

$$
\operatorname{Ker} \Delta_{\omega}(\omega)=\mathcal{K}
$$

The rest of the proof is broken into steps.

Step 1. Let $\omega \in \mathbb{D}, \mu \in \mathbb{T}, f \in \operatorname{Ker} P^{\frac{1}{2}} G(\mu)$ and let $G(\mu)$ be invertible. Then

$$
C f=0 \quad \Longleftrightarrow \quad \Delta_{\omega}(\omega) f=0 .
$$

Proof of Step 1: Since $\rho_{\omega}(\omega)>0, \Delta_{\omega}(\omega) f=0$ implies $C_{1} f=0$ and $C_{2} f=0$ by (6.1) and (6.2). To prove the opposite implication, let $C f=0$. Then, by (1.10),

$$
M^{*} P M f-N^{*} P N f=0 .
$$

By assumption, $f \in \operatorname{Ker} P^{\frac{1}{2}} G(\mu)$, i.e.,

$$
P^{\frac{1}{2}} M f=\mu P^{\frac{1}{2}} N f
$$


which enables us to rewrite (6.15) as $G(\mu)^{*} P M f=0$. Since $G(\mu)$ is assumed to be invertible, this implies that $P^{\frac{1}{2}} M f=0$ and hence from (6.16) that also $P^{\frac{1}{2}} N f=0$. Thus, by (6.1), $\Delta_{\omega}(\omega) f=0$.

Step 2. Let $\omega \in \mathbb{D}, \mu \in \mathbb{T}$ and let $G(\mu)$ be invertible. Then

$$
\operatorname{dim} \operatorname{Ker} P^{\frac{1}{2}} G(\mu)=\operatorname{dim} \mathcal{K}+\operatorname{dim} \mathcal{R},
$$

where $\mathcal{R}$ and $\mathcal{K}$ are the subspaces of $\mathbb{C}^{p+q}$ and $\mathbb{C}^{n}$ that are defined in (6.12) and (6.5), respectively.

Proof of Step 2: By definition $(6.5), \mathcal{K} \subseteq \operatorname{Ker} P^{\frac{1}{2}} G(\mu)$. Let

$$
\operatorname{Ker} P^{\frac{1}{2}} G(\mu)=\mathcal{K}+\mathcal{V}
$$

be any direct sum decomposition of $\operatorname{Ker} P^{\frac{1}{2}} G(\mu)$. Let $\left\{f_{1}, \ldots, f_{k}\right\}$ and $\left\{g_{1}, \ldots, g_{m}\right\}$ be bases of $\mathcal{K}$ and $\mathcal{V}$, respectively. Since $G(\mu)$ is invertible, the vectors

$$
\left\{G(\mu) f_{j}\right\}_{j=1}^{k} \text { and }\left\{G(\mu) g_{\ell}\right\}_{\ell=1}^{m}
$$

form a basis of Ker $P$ and therefore, $k+m=n-\operatorname{rank} P$. By definition (6.12),

$$
\mathcal{R}=\operatorname{span}\left\{C G^{-1}(\mu) G(\mu) f_{j}, \quad C G^{-1}(\mu) G(\mu) g_{\ell}\right\} .
$$

Since $f_{j} \in \mathcal{K}, C f_{j}=0$ and therefore,

$$
\mathcal{R}=\operatorname{span}\left\{C g_{\ell}\right\}_{\ell=1}^{m}
$$

Moreover, the vectors $C g_{1}, \ldots C g_{m}$ are linearly independent because if

$$
0=\sum_{\ell=1}^{m} \alpha_{\ell} C g_{\ell}=C\left(\sum_{\ell=1}^{m} \alpha_{\ell} g_{\ell}\right)
$$

then the vector $g=\sum_{\ell=1}^{m} \alpha_{\ell} g_{\ell}$ belongs to $\mathcal{K} \cap \mathcal{V}=\{0\}$. Since the $g_{\ell}$ are linearly independent, this implies that $\alpha_{\ell}=0$ for all $\ell$. Therefore, $\operatorname{dim} \mathcal{V}=\operatorname{dim} \mathcal{R}$, which together with (6.18) implies (6.17).

Finally, rewriting (6.17) as

$$
n-\operatorname{rank} P=n-\operatorname{rank} \Delta_{\omega}(\omega)+\operatorname{dim} \mathcal{R}
$$

we get (6.13) for the case $\omega \in \mathbb{D}$, which completes the proof of lemma.

Now we summarize some implications of the last three lemmas for the particular case when $P$ is strictly positive.

Lemma 6.5 Let $P$ be a strictly positive solution of the Lyapunov-Stein equation (1.10) and suppose that either $\omega \in \mathbb{D}$ or $\omega \in \mathbb{T}$ and $G(\omega)$ is invertible. Then $\Delta_{\omega}(z)$ is invertible for all $z \in \mathbb{D}$ and for all $z \in \mathbb{T}$ at which $G(z)$ is invertible. 
Proof: Since $P$ is invertible and det $G(z) \not \equiv 0$, it follows from $(6.5)$ that $\mathcal{K}=\{0\}$. Therefore the asserted invertibility follows from Lemma 6.2 (if $\omega \in \mathbb{D}$ ) and from Lemma 6.3 (if $z \neq$ $\omega \in \mathbb{T}$ and $G(\omega)$ is invertible). It remains to show that $\Delta_{\omega}(\omega)$ is also invertible for $\omega \in \mathbb{T}$ when $G(\omega)$ is invertible. But this follows immediately from (6.13).

¿From now on, let $\operatorname{dim} \mathcal{K}=k$ and let $\mathrm{Q} \in \mathbb{C}^{n \times(n-k)}$ be an isometric matrix whose columns span $\mathcal{K}^{\perp}$, the orthogonal complement of $\mathcal{K}$ in $\mathbb{C}^{n}$ with respect to the standard inner product.

Lemma 6.6 If $P \geq 0$ is a solution of the Lyapunov-Stein equation (1.10) and $\omega \in \mathbb{D}$, then $\mathbf{Q}^{*} \Delta_{\omega}(z) \mathbf{Q}$ is invertible for every point $z \in \mathbb{D}$ and for every point $z \in \mathbb{I}$ at which $G(z)$ is invertible.

Proof: Let $\mathbf{Q}^{*} \Delta_{\omega}(z) \mathbf{Q} u=0$ for some $u \in \mathbb{C}^{n-k}$. Then

$$
u^{*} \mathbf{Q}^{*}\left\{\rho_{\omega}(z)^{*} \Delta_{\omega}(z)+\rho_{\omega}(z) \Delta_{\omega}(z)^{*}\right\} \mathbf{Q} u=0
$$

and hence, upon invoking formula (6.4), it follows just as in the proof of Lemma 6.2 that $\mathbf{Q} u \in \mathcal{K}$. Therefore, since $\mathbf{Q} u \in \mathcal{K}^{\perp}$ by definition, we see that $\mathbf{Q} u=0$ and

$$
u=\mathbf{Q}^{*} \mathbf{Q} u=0
$$

This completes the proof, since $\mathbf{Q}^{*} \Delta_{\omega}(z) \mathbf{Q}$ is a square matrix.

We now define

$$
\Delta_{\omega}^{[-1]}(z):=\mathbf{Q}\left(\mathbf{Q}^{*} \Delta_{\omega}(z) \mathbf{Q}\right)^{-1} \mathbf{Q}^{*}
$$

for all points $z \in \mathbb{C}$ at which the indicated inverse exists. Since $\operatorname{det}\left\{\mathbf{Q}^{*} \Delta_{\omega}(z) \mathbf{Q}\right\}$ is a polynomial in $z$ of degree at most $n-k$, which has no zeros in $\mathbb{D}$ by the preceding lemma, the inverse can fail to exist at most at $n-k$ points, all of which fall outside $\mathbb{D}$. The next conclusion now follows immediately from Lemma 6.6.

Remark 6.7 If $P \geq 0$ is a solution of the Lyapunov-Stein equation (1.10) and $\omega \in \mathbb{D}$, then the function $\Delta_{\omega}^{[-1]}(z)$ is rational and has at most $n-k$ poles all of which fall outside $\mathbb{D}$. Moreover, it is analytic at every point $z \in \mathbb{T}$ at which $G(z)$ is invertible.

Lemma 6.8 If $P \geq 0$ is a solution of the Lyapunov-Stein equation (1.10) and $\omega \in \mathbb{D}$, then the resolventlike identities

$$
\begin{aligned}
\rho_{\omega}(\omega) \Delta_{\omega}^{[-1]}(\omega)-\rho_{\omega}(z) \Delta_{\omega}^{[-1]}(z) & =\delta_{\omega}(z) \Delta_{\omega}^{[-1]}(\omega) G(\omega)^{*} P H(\omega)^{*} \Delta_{\omega}^{[-1]}(z) \\
& =\delta_{\omega}(z) \Delta_{\omega}^{[-1]}(z) G(\omega)^{*} P H(\omega)^{*} \Delta_{\omega}^{[-1]}(\omega)
\end{aligned}
$$

hold for every point $z$ at which $\Delta_{\omega}^{[-1]}(z)$ is analytic.

Proof: In view of (6.1), (1.4) and (1.15),

$$
\rho_{\omega}(\omega) \Delta_{\omega}(z)-\rho_{\omega}(z) \Delta_{\omega}(\omega)=\delta_{\omega}(z) G(\omega)^{*} P H(\omega)^{*},
$$


which together with (6.19) implies the first equality in (6.20):

$$
\begin{aligned}
& \rho_{\omega}(\omega) \Delta_{\omega}^{[-1]}(\omega)-\rho_{\omega}(z) \Delta_{\omega}^{[-1]}(z) \\
& =\rho_{\omega}(\omega) \mathbf{Q}\left(\mathbf{Q}^{*} \Delta_{\omega}(\omega) \mathbf{Q}\right)^{-1} \mathbf{Q}^{*}-\rho_{\omega}(z) \mathbf{Q}\left(\mathbf{Q}^{*} \Delta_{\omega}(z) \mathbf{Q}\right)^{-1} \mathbf{Q}^{*} \\
& =\mathbf{Q}\left(\mathbf{Q}^{*} \Delta_{\omega}(\omega) \mathbf{Q}\right)^{-1} \mathbf{Q}^{*}\left(\rho_{\omega}(\omega) \Delta_{\omega}(z)-\rho_{\omega}(z) \Delta_{\omega}(\omega)\right) \mathbf{Q}\left(\mathbf{Q}^{*} \Delta_{\omega}(z) \mathbf{Q}\right)^{-1} \mathbf{Q}^{*} \\
& =\delta_{\omega}(z) \Delta_{\omega}^{[-1]}(\omega) G(\omega)^{*} P H(\omega)^{*} \Delta_{\omega}^{[-1]}(z) .
\end{aligned}
$$

The second equality is checked in much the same way.

Lemma 6.9 If $P \geq 0$ is a solution of the Lyapunov-Stein equation (1.10) and $\omega \in \mathbb{D}$, then the following formulas hold at every point $z \in \overline{\mathbb{D}}$ at which $\Delta_{\omega}^{[-1]}(z)$ is analytic:

(1) $\Delta_{\omega}^{[-1]}(z) \Delta_{\omega}(z) \Delta_{\omega}^{[-1]}(z)=\Delta_{\omega}^{[-1]}(z)$,

(2) $\Delta_{\omega}(z) \Delta_{\omega}^{[-1]}(z) \Delta_{\omega}(z)=\Delta_{\omega}(z)$,

(3) $\Delta_{\omega}(z) \Delta_{\omega}^{[-1]}(z)=\Delta_{\omega}^{[-1]}(z) \Delta_{\omega}(z)=I_{n}-\mathbf{P}_{\mathcal{K}}$,

where $\mathbf{P}_{\mathcal{K}}$ denotes the orthogonal projection of $\mathbb{C}^{n}$ onto $\mathcal{K}$.

Proof: The first assertion is an immediate consequence of (6.19), whereas the second is easily verified upon representing an arbitrary vector $u \in \mathbb{C}^{n}$ as

$$
u=y+\mathbf{Q} x \quad \text { with } \quad x \in \mathbb{C}^{n-k} \text { and } y \in \mathcal{K} .
$$

Indeed, using (6.25) and taking advantage of (6.7) we get

$$
\begin{aligned}
\Delta_{\omega}(z)\left(I_{n}-\Delta_{\omega}^{[-1]}(z) \Delta_{\omega}(z)\right) u & =\Delta_{\omega}(z)\left(I_{n}-\Delta_{\omega}^{[-1]}(z) \Delta_{\omega}(z)\right)(y+\mathbf{Q} x) \\
& =\Delta_{\omega}(z)\left(I_{n}-\Delta_{\omega}^{[-1]}(z) \Delta_{\omega}(z)\right) y=0
\end{aligned}
$$

which is equivalent to (6.23). It follows from (6.22) and (6.23) that

$$
\left(\Delta_{\omega}^{[-1]}(z) \Delta_{\omega}(z)\right)^{2}=\Delta_{\omega}^{[-1]}(z) \Delta_{\omega}(z) \quad \text { and } \quad\left(\Delta_{\omega}(z) \Delta_{\omega}^{[-1]}(z)\right)^{2}=\Delta_{\omega}(z) \Delta_{\omega}^{[-1]}(z),
$$

which means that the operators $\Delta_{\omega}^{[-1]}(z) \Delta_{\omega}(z)$ and $\Delta_{\omega}(z) \Delta_{\omega}^{[-1]}(z)$ are projections. Since the matrix $\mathbf{Q}$ is isometric, the representation (6.25) of $u \in \mathbb{C}^{n}$ is orthogonal and thus, the equality

$$
\Delta_{\omega}^{[-1]}(z) \Delta_{\omega}(z)(y+\mathbf{Q} x)=\mathbf{Q} x
$$

implies that $\Delta_{\omega}^{[-1]}(z) \Delta_{\omega}(z)$ is the orthogonal projection of $\mathbb{C}^{n}$ onto $\operatorname{Ran} \mathbf{Q}=\mathcal{K}^{\perp}$, which proves the second equality in (6.24). Similarly, the equality

$$
\Delta_{\omega}^{[-1]}(z)^{*} \Delta_{\omega}(z)^{*}(y+\mathbf{Q} x)=\mathbf{Q} x
$$

implies that $\Delta_{\omega}^{[-1]}(z) \Delta_{\omega}(z)$ is also the orthogonal projection of $\mathbb{C}^{n}$ onto $\mathcal{K}^{\perp}$. Thus,

$$
\Delta_{\omega}^{[-1]}(z)^{*} \Delta_{\omega}(z)^{*}=I_{n}-\mathbf{P}_{\mathcal{K}}
$$

and the proof of lemma is completed upon taking adjoints in the last equality. 
In view of the identities

$$
\Delta_{\omega}(z) \Delta_{\omega}^{[-1]}(z)=\left(\Delta_{\omega}(z) \Delta_{\omega}^{[-1]}(z)\right)^{*} \quad \text { and } \quad \Delta_{\omega}^{[-1]}(z) \Delta_{\omega}(z)=\left(\Delta_{\omega}^{[-1]}(z) \Delta_{\omega}(z)\right)^{*},
$$

which are immediate from (6.24), and the identities (6.22) and (6.23), $\Delta_{\omega}^{[-1]}(z)$ is the MoorePenrose pseudoinverse (see e.g. [?, Section 12.8]) of $\Delta_{\omega}(z)$ for every point $z \in \overline{\mathbb{D}}$ at which $\Delta_{\omega}^{[-1]}(z)$ is analytic.

The function $\Delta_{\omega}^{[-1]}(z)$ is also meaningful if $\omega \in \mathbb{T}$ and $G(\omega)$ is invertible. Moreover, it is analytic inside the unit disc. However, we cannot guarantee that it has no poles on the unit circle and this motivates the introduction of a different pseudoinverse of $\Delta_{\omega}$.

Lemma 6.10 Let $P \geq 0$ be a solution of the Lyapunov-Stein equation (1.10), let $r=\operatorname{rank} P$ and let $Q \in \mathbb{C}^{n \times r}, M_{\diamond} \in \mathbb{C}^{r \times r}$ and $N_{\diamond} \in \mathbb{C}^{r \times r}$ be matrices satisfying (5.1) and (5.14). Furthermore, let $\mu \in \mathbb{T}$ be such that $G(\mu)$ is invertible. Then the $n \times n$ matrix valued rational function

$$
\Delta_{\mu}^{\dagger}(z):=Q\left(Q^{*} \Delta_{\mu}(z) Q\right)^{-1} Q^{*}
$$

has at most $r$ poles but is analytic in $\mathbb{D}$ and at every point on $\mathbb{T}$ at which $G$ is invertible.

Proof: Let

$$
\widetilde{C}=\left(\begin{array}{c}
\widetilde{C}_{1} \\
\widetilde{C}_{2}
\end{array}\right)=C Q \text { and } \widetilde{P}=Q^{*} P Q
$$

By (5.1) and (5.14), $\widetilde{P}$ is strictly positive and satisfies the Lyapunov-Stein equation

$$
M_{\diamond}^{*} \widetilde{P} M_{\diamond}-N_{\diamond}^{*} \widetilde{P} N_{\diamond}=\widetilde{C} J \widetilde{C} .
$$

Furthermore, (5.14) implies that

$$
\begin{aligned}
Q^{*} \Delta_{\mu}(z) Q & =Q^{*}\left\{G(\mu)^{*} P G(z)+\rho_{\mu}(z) C_{2}^{*} C_{2}\right\} Q \\
& =\left(M_{\diamond}-\mu N_{\diamond}\right)^{*} \widetilde{P}\left(M_{\diamond}-\mu N_{\diamond}\right)+\rho_{\mu}(z) \widetilde{C}_{2}^{*} \widetilde{C}_{2} .
\end{aligned}
$$

Since $G(\mu)$ is invertible, $M_{\diamond}-\mu N_{\diamond}$ is also invertible (by Remark 5.5). Therefore, Lemma 6.5 is applicable to the $r \times r$ matrix valued function $Q^{*} \Delta_{\mu}(z) Q$ and guarantees that det $\left(Q^{*} \Delta_{\mu}(z) Q\right) \neq$ 0 for all $z \in \mathbb{D}$ and for all $z \in \mathbb{T}$ at which $G(z)$ is invertible. Finally, $\Delta_{\mu}^{\dagger}(z)$ has at most $r$ poles, since $\operatorname{det}\left(Q^{*} \Delta_{\mu}(z) Q\right)$ is a polynomial of degree at most $r$.

We remark that in general, $\Delta_{\mu}^{\dagger}(z)$ is only a "semipseudoinverse" of $\Delta_{\mu}(z)$ : the equality

$$
\Delta_{\mu}^{\dagger}(z) \Delta_{\mu}(z) \Delta_{\mu}^{\dagger}(z)=\Delta_{\mu}^{\dagger}(z)
$$

holds for every point $z \in \overline{\mathbb{D}}$ at which $\Delta_{\mu}^{\dagger}$ exists, whereas

$$
\Delta_{\mu}(z) \Delta_{\mu}^{\dagger}(z) \Delta_{\mu}(z) x=\Delta_{\mu}(z) x
$$

holds only for $x \in \operatorname{Ran} Q+\operatorname{Ker} \Delta_{\mu}(z)$. This subspace is not equal to $\mathbb{C}^{n}$ when $z \in \mathbb{D}$. However, if $z=\mu$, then $\operatorname{Ran} Q+\operatorname{Ker} \Delta_{\mu}(\mu)=\mathbb{C}^{n}$ and $\Delta_{\mu}^{\dagger}(\mu)$ is a pseudoinverse of $\Delta_{\mu}(\mu)$. 


\section{Description of all solutions}

In this section we describe the set of all solutions $S$ of the FMI (3.4) when $P \in \mathbb{C}^{n \times n}$ is a positive semidefinite solution of the Lyapunov-Stein equation (1.8) with $\operatorname{rank} P=r \leq n$.

In view of the analysis in Section 5 (which is summarized in Remark 5.4) we can assume without loss of generality that there exists a matrix $Q \in \mathbb{C}^{n \times r}$ such that

$$
Q^{*} P Q>0, \quad \operatorname{rank} Q^{*} P Q=\operatorname{rank} P=r
$$

and

$$
M Q=Q M_{\diamond} \text { and } \quad N Q=Q N_{\diamond}
$$

for some matrices $M_{\diamond}, N_{\diamond} \in \mathbb{C}^{r \times r}$ satisfying the spectral conditions (5.13). Let

$$
P^{[-1]}=Q\left(Q^{*} P Q\right)^{-1} Q^{*} \text {. }
$$

The next lemma serves to identify $P^{[-1]}$ as a pseudoinverse of $P$.

Lemma 7.1 For any $Q \in \mathbb{C}^{n \times r}$ satisfying the condition (7.1), the matrix $P^{[-1]}$ defined via (7.3) satisfies the identities

$$
P^{[-1]} P P^{[-1]}=P^{[-1]} \quad \text { and } \quad P P^{[-1]} P=P,
$$

whereas $I_{n}-P^{[-1]} P$ is the projection of $\mathbb{C}^{n}$ onto $\operatorname{Ker} P$ along the subspace $\mathcal{Q}=\operatorname{Ran} Q$.

Proof: The proof is similar to the proof of Lemma 6.9. The first equality in (7.4) follows easily from the definition (7.3) of $P^{[-1]}$. Next, in view of (5.16), every vector $u \in \mathbb{C}^{n}$ can be decomposed as

$$
f=y+Q x \quad \text { for some } \quad y \in \operatorname{Ker} P \quad \text { and } \quad x \in \mathbb{C}^{r}
$$

Therefore,

$$
P\left(I_{n}-P^{[-1]} P\right) u=P\left(I_{n}-P^{[-1]} P\right)(y+Q x)=P\left(I_{n}-P^{[-1]} P\right) y=0,
$$

which is equivalent to the second equality in (7.4). Finally, (7.4) and (7.5) imply that

$$
\left(I_{n}-P^{[-1]} P\right)(y+Q x)=y \quad \text { and } \quad\left(I_{n}-P^{[-1]} P\right)^{2}=I_{n}-P^{[-1]} P,
$$

and thus, $I_{n}-P^{[-1]} P$ is the projection onto $\operatorname{Ker} P$ parallel to the range of $Q$.

Note that in general, the matrix $P^{[-1]}$ defined via (7.4) is not the Moore-Penrose pseudoinverse, since the matrices $P^{[-1]} P$ and $P P^{[-1]}$ are not necessarily Hermitian.

Lemma 7.2 Let assumptions (7.1) and (7.2) be in force and let $\mu \in \mathbb{T}$ be such that $G(\mu)$ is invertible. Then the $\mathbb{C}^{(p+q) \times(p+q)}$-valued function $\Theta$ given by

$$
\Theta(z)=I_{p+q}-\rho_{\mu}(z) C G^{-1}(z) P^{[-1]} G(\mu)^{-*} C^{*} J
$$

is $J$-inner in the unit disc and, moreover,

$$
J-\Theta(z) J \Theta(w)^{*}=\rho_{w}(z) C G^{-1}(z) P^{[-1]} G(w)^{-*} C^{*}
$$

for every pair of points $z$ and $w$ in $\mathcal{A}_{\Theta}$, the domain of analyticity of $\Theta$. 
Proof: The assumptions (7.1) and (7.2) enable us to reduce the case of positive semidefinite $P$ to the strictly positive case: in view of $(7.2)$,

$$
G^{-1}(z) Q=Q\left(M_{\diamond}-z N_{\diamond}\right)^{-1}
$$

which, on account of (7.3), allows us to reexpress formula (7.6) as

$$
\Theta(z)=I_{p+q}-\rho_{\mu}(z) \widetilde{C}\left(M_{\diamond}-z N_{\diamond}\right)^{-1} \widetilde{P}^{-1}\left(M_{\diamond}-\mu N_{\diamond}\right)^{-*} \widetilde{C}^{*} J
$$

where $\widetilde{C}$ and $\widetilde{P}$ are the matrices defined by (6.27). By (7.1) and (7.2), $\widetilde{P}$ is strictly positive and satisfies the Lyapunov-Stein equation (6.28) But, as is well known (see e.g., [16, Section $9]$ ), a function $\Theta$ of the form (7.9) is $J$-inner in the unit disc and

$$
J-\Theta(z) J \Theta(w)^{*}=\rho_{w}(z) \widetilde{C}\left(M_{\diamond}-z N_{\diamond}\right)^{-1} \widetilde{P}^{-1}\left(M_{\diamond}-w N_{\diamond}\right)^{-*} \widetilde{C}^{*} .
$$

It remains only to note that the expressions on the right hand sides of (7.7) and (7.10) coincide, in view of (7.3) and (7.8).

Corollary 7.3 Let $\Theta$ be the function given by (7.6) and let

$$
\Theta=\left(\begin{array}{ll}
\theta_{11} & \theta_{12} \\
\theta_{21} & \theta_{22}
\end{array}\right):\left(\begin{array}{l}
\mathbb{C}^{p} \\
\mathbb{C}^{q}
\end{array}\right) \rightarrow\left(\begin{array}{l}
\mathbb{C}^{p} \\
\mathbb{C}^{q}
\end{array}\right)
$$

be its partition into four blocks of the indicated sizes. Then the rational function $\theta_{22}^{-1} \theta_{21}$ belongs to $\mathcal{S}^{p \times q}$. Moreover, $\theta_{22}^{-1}(z) \theta_{21}(z)$ is strictly contractive for all $z \in \mathbb{D}$ and for every $z \in \mathbb{T}$ at which $G(z)$ is invertible.

Proof: By (7.6), the mvf $\Theta$ is rational and analytic at every point $z$ at which $G(z)$ is invertible. Next, $\Theta(\mu)=I_{p+q}$ and in particular, $\theta_{21}(\mu)=0$ and $\theta_{22}(\mu)=I_{q}$. It follows from (7.7) that for every point $z \in \mathcal{A}_{\Theta} \cap \overline{\mathrm{D}}$,

$$
\theta_{22}(z) \theta_{22}(z)^{*} \geq I_{q}+\theta_{21}(z) \theta_{21}(z)^{*}
$$

and thus, as $\operatorname{det} \theta_{22} \not \equiv 0$, the function $\widetilde{\theta}:=\theta_{22}^{-1} \theta_{21}$ is well defined and

$$
\widetilde{\theta}(z) \tilde{\theta}(z)^{*} \leq I_{p}-\theta_{22}^{-1}(z) \theta_{22}(z)^{-*} \quad \text { for every point } z \in \mathcal{A}_{\Theta} \cap \overline{\mathbb{D}}
$$

Therefore, the singularities of $\tilde{\theta}$ in $\mathbb{D}$ are removable, $\tilde{\theta} \in \mathcal{S}^{p \times q}$ and $\tilde{\theta}(z)$ is strictly contractive at every point $z \in \mathcal{A}_{\Theta} \cap \overline{\mathbb{D}}$ and in particular, at every point $z \in \mathbb{T}$ at which $G(z)$ is invertible. To show this, suppose that $\widetilde{\theta}\left(z_{0}\right)$ is not strictly contractive for some $z_{0} \in \mathbb{D}$, i.e., that

$$
\widetilde{\theta}\left(z_{0}\right) x=y \quad \text { for } \quad x \in \mathbb{C}^{p} \quad \text { and } \quad y \in \mathbb{C}^{q} \quad \text { such that } \quad x^{*} x=y^{*} y \neq 0 .
$$

By the maximum principle, $\tilde{\theta}(z) x=y$ for all $z \in \mathbb{D}$ and so too, by continuity, $\tilde{\theta}(\mu) x=y$, which is impossible, since $\widetilde{\theta}(\mu)=0$.

Now we pass to the main result of this section. 
Theorem 7.4 Let (1.5) and (7.2) be in force and let $P \in \mathbb{C}^{n \times n}$ be a positive semidefinite solution of the Lyapunov-Stein equation (1.8) with $\operatorname{rank} P=r$. Let $\theta_{j k}$ be the block entries from the decomposition (7.11) of the mvf $\Theta$ defined in (7.6). Then all the solutions $S$ of the $\operatorname{aBIP}(M, N, P, C)$ are parametrized by the linear fractional transformation

$$
S(z)=\left(\theta_{11}(z) \mathcal{E}(z)+\theta_{12}(z)\right)\left(\theta_{21}(z) \mathcal{E}(z)+\theta_{22}(z)\right)^{-1},
$$

in which the parameter $\mathcal{E} \in \mathcal{S}^{p \times q}$ is of the form

$$
\mathcal{E}(z)=U\left(\begin{array}{cc}
\widehat{\mathcal{E}}(z) & 0 \\
0 & I_{\nu}
\end{array}\right) V
$$

where $U \in \mathbb{C}^{p \times p}$ and $V \in \mathbb{C}^{q \times q}$ are fixed unitary matrices which depend only on the interpolation data, $\nu$ is the integer given by $(1.11)$ and $\widehat{\mathcal{E}}(z)$ is an arbitrary $\mathbb{C}^{(p-\nu) \times(q-\nu)}$-valued analytic contraction in $\mathbb{D}$ (i.e., $\left.\widehat{\mathcal{E}} \in \mathcal{S}^{(p-\nu) \times(q-\nu)}\right)$.

Proof: In view of Theorem 3.7, it suffices to show that the transformation (7.13) with $\mathcal{E}$ as in (7.14) parametrizes all the solutions of the FMI (3.4). By Corollary 7.3, the function $\theta_{22}^{-1} \theta_{21}$ is strictly contractive in $\mathbb{D}$. Therefore for every $\mathcal{E} \in \mathcal{S}^{p \times q}$,

$$
\operatorname{det}\left(\theta_{21} \mathcal{E}+\theta_{22}\right)=\operatorname{det} \theta_{22}^{-1}(z) \operatorname{det}\left(\theta_{22}^{-1} \theta_{21} \mathcal{E}+I_{q}\right) \not \equiv 0
$$

and therefore, the transformation (7.13) is well defined.

In view of (5.16), the FMI (3.4) is equivalent to the inequality

$$
\left(\begin{array}{cc}
Q^{*} P Q & Q^{*} G(z)^{-*} C^{*}\left(\begin{array}{c}
I_{p} \\
-S(z)^{*}
\end{array}\right) \\
\left(I_{p},-S(z)\right) C G(z)^{-1} Q & \frac{I_{p}-S(z) S(z)^{*}}{\rho_{z}(z)}
\end{array}\right) \geq 0
$$

together with the condition

$$
\left(I_{p},-S(z)\right) C G(z)^{-1} \mathbf{P}_{\mathrm{Ker} P} \equiv 0,
$$

where $\mathbf{P}_{\text {Ker } P}$ is the orthogonal projection of $\mathbb{C}^{n}$ onto Ker $P$. The rest of the proof is divided into the following three steps:

Step 1. All solutions $S$ of the inequality (7.15) are parametrized by (7.13), where the parameter $\mathcal{E}$ varies over $\mathcal{S}^{p \times q}$.

Step 2. A mvf $S \in \mathcal{S}^{p \times q}$ of the form (7.13) satisfies (7.16) if and only if the corresponding parameter $\mathcal{E}$ is subject to

$$
\left(I_{p},-\mathcal{E}(z)\right) C G(\mu)^{-1} \mathbf{P}_{\text {KerP }} \equiv 0 .
$$

Step 3. A function $\mathcal{E}$ satisfies (7.17) if and only if it is of the form (7.14).

Proof of Step 1. Since $Q^{*} P Q>0$, the inequality (7.15) is equivalent to

$$
\left(I_{p},-S(z)\right)\left\{\frac{J}{\rho_{z}(z)}-C G(z)^{-1} P^{[-1]} G(z)^{-*} C^{*}\right\}\left(\begin{array}{c}
I_{p} \\
-S(z)^{*}
\end{array}\right) \geq 0
$$


On account of (7.7), the last inequality can be written as

$$
\left(I_{p},-S(z)\right) \frac{\Theta(z) J \Theta(z)^{*}}{\rho_{z}(z)}\left(\begin{array}{c}
I_{p} \\
-S(z)^{*}
\end{array}\right) \geq 0 .
$$

But it is well known that this is equivalent to the assertion of Step 1 (see e.g., [13, Theorem 3.8]).

Proof of Step 2. Let $S$ be of the form (7.13). Then

$$
\left(I_{p},-S(z)\right) \Theta(z)=\left(\theta_{11}(z)-S(z) \theta_{21}(z)\right)\left(I_{p},-\mathcal{E}(z)\right) .
$$

Substituting the latter relation into (7.16) we obtain

$$
\left(I_{p},-\mathcal{E}(z)\right) \Theta^{-1}(z) C G(z)^{-1} \mathbf{P}_{\mathrm{Ker} P} \equiv 0 .
$$

It follows from (7.7) that $\Theta$ is $J$-unitary on the unit circle and in particular, that it satisfies the symmetry relation

$$
\Theta(z)^{-1}=J \Theta(1 / \bar{z})^{*} J
$$

which together with (7.6) and the identities

$$
z G(1 / \bar{z})^{*}=H(z), \quad z \rho_{\mu}(1 / \bar{z})^{*}=\delta_{\mu}(z) \quad(z \neq 0)
$$

leads to

$$
\Theta^{-1}(z)=I_{p+q}-\delta_{\mu}(z) C G(\mu)^{-1} P^{[-1]} H(z)^{-1} C^{*} J
$$

Using the identity

$$
H(z)^{-1} C^{*} J C G(z)^{-1}=H(z)^{-1} M^{*} P+P N G(z)^{-1},
$$

which follows immediately from (1.8), we obtain

$$
\begin{aligned}
\Theta^{-1}(z) C G(z)^{-1} & =\left(I_{p+q}-\delta_{\mu}(z) C G(\mu)^{-1} P^{[-1]} H(z)^{-1} C^{*} J\right) C G(z)^{-1} \\
& =C G(z)^{-1}-\delta_{\mu}(z) C G(\mu)^{-1} P^{[-1]}\left(H(z)^{-1} M^{*} P+P N G(z)^{-1}\right)
\end{aligned}
$$

Substituting the latter equality into (7.18) we get

$$
\left(I_{p},-\mathcal{E}(z)\right) C\left(I_{p+q}-\delta_{\mu}(z) G(\mu)^{-1} P^{[-1]} P N\right) G(z)^{-1} \mathbf{P}_{\mathrm{Ker} P} \equiv 0 .
$$

Since

$$
G(\mu)-\delta_{\mu}(z) P^{[-1]} P N=G(z)+\delta_{\mu}(z)\left(I-P^{[-1]} P\right) N,
$$

one can rewrite (7.19) as

$$
\left(I_{p},-\mathcal{E}(z)\right) C G(\mu)^{-1}\left\{I_{p+q}+\delta_{\mu}(z)\left(I-P^{[-1]} P\right) N G(z)^{-1}\right\} \mathbf{P}_{\mathrm{Ker} P} \equiv 0 .
$$

It was mentioned in the proof of Lemma 7.1 that $I-P^{[-1]} P$ is a projection (not orthogonal, in general) onto $\operatorname{Ker} P$. Therefore

$$
\left(I-P^{[-1]} P\right)=\mathbf{P}_{\mathrm{Ker} P}\left(I-P^{[-1]} P\right)
$$


and the condition (7.20) is equivalent to

$$
\left(I_{p},-\mathcal{E}(z)\right) C G(\mu)^{-1} \mathbf{P}_{\operatorname{Ker} P}\left(I_{p+q}+\delta_{\mu}(z)\left(I-P^{[-1]} P\right) N G(z)^{-1} \mathbf{P}_{\operatorname{Ker} P}\right) \equiv 0 .
$$

Since the mvf

$$
I_{p+q}+\delta_{\mu}(z)\left(I-P^{[-1]} P\right) N G(z)^{-1} \mathbf{P}_{\operatorname{Ker} P}
$$

is invertible everywhere except for at most finitely many points $z$ (it is a rational function taking the value $I_{p+q}$ at the point $\mu$ ), conditions (7.21) and (7.17) are equivalent.

Proof of Step 3. It follows from (1.8) that

$$
\begin{aligned}
G(\mu)^{-*} C^{*} J C G(\mu)^{-1} & =G(\mu)^{-*} M^{*} P M G(\mu)^{-1}-G(\mu)^{-*} N^{*} P N G(\mu)^{-1} \\
& =\left(I_{n}+\bar{\mu} G(\mu)^{-*} N^{*}\right) P\left(I_{n}+\mu N G(\mu)^{-1}\right)-G(\mu)^{-*} N^{*} P N G(\mu)^{-1} \\
& =P+\bar{\mu} G(\mu)^{-*} N^{*} P+\mu P N G(\mu)^{-1} .
\end{aligned}
$$

Multiplying both sides of the latter equality by $\mathbf{P}_{\text {Ker } P}$ from the right and from the left we get

$$
\mathbf{P}_{\text {Ker } P} G(\mu)^{-*} C^{*} J C G(\mu)^{-1} \mathbf{P}_{\text {KerP }}=0 .
$$

and hence that the subspace $\mathcal{R}$ of $\mathbb{C}^{p+q}$ which was defined in (6.12) is $J$-neutral. It is readily checked by standard arguments that the subspaces

$$
\mathcal{R}_{1}:=\operatorname{Ran} C_{1} G(\mu)^{-1} \mathbf{P}_{\operatorname{Ker} P}=\left\{C_{1} G(\mu)^{-1} g, \quad g \in \operatorname{Ker} P\right\} \subseteq \mathbb{C}^{p}
$$

and

$$
\mathcal{R}_{2}:=\operatorname{Ran} C_{2} G(\mu)^{-1} \mathbf{P}_{\operatorname{Ker} P}=\left\{C_{2} G(\mu)^{-1} g, \quad g \in \operatorname{Ker} P\right\} \subseteq \mathbb{C}^{q}
$$

have the same dimensions as $\mathcal{R}$. Indeed, in view of (7.22), every vector $x \in \mathcal{R}$ can be represented as $x=\left(\begin{array}{c}\xi \\ \eta\end{array}\right)$ for some $\xi \in \mathcal{R}_{1}$ and $\eta \in \mathcal{R}_{2}$ such that $\|\xi\|=\|\eta\|$. Let the vectors

$$
x_{j}=\left(\begin{array}{c}
\xi_{j} \\
\eta_{j}
\end{array}\right) \in \mathcal{R} \quad(j=1, \ldots, k)
$$

be linearly independent, let $\eta:=\sum_{j=1}^{k} \alpha_{j} \eta_{j}=0$ for some choice of $\alpha_{j} \in \mathbb{C}$ and let

$$
x=\sum_{j=1}^{k} \alpha_{j} x_{j}=\left(\begin{array}{l}
\xi \\
0
\end{array}\right) .
$$

Then, as $x \in \mathcal{R},\|\xi\|=\|\eta\|=0$. Thus, $x=0$ and $\alpha_{j}=0$ for all $j=1, \ldots, k$. This shows that the vectors $\eta_{j}$ are linearly independent and therefore, that $\operatorname{dim} \mathcal{R} \leq \operatorname{dim} \mathcal{R}_{2}$. Thus, as the opposite inequality is selfevident, $\operatorname{dim} \mathcal{R}=\operatorname{dim} \mathcal{R}_{2}$ and, similarly, $\operatorname{dim} \mathcal{R}=\operatorname{dim} \mathcal{R}_{1}$. The condition (7.17), rewritten with help of (1.9) as

$$
\mathcal{E}(z) C_{2} G(\mu)^{-1} \mathbf{P}_{\mathrm{Ker} P} \equiv C_{1} G(\mu)^{-1} \mathbf{P}_{\mathrm{Ker} P},
$$


displays the fact that the Schur function $\mathcal{E} \in \mathcal{S}^{p \times q}$ maps $\mathcal{R}_{2}$ isometrically onto $\mathcal{R}_{1}$ for every $z \in \mathbb{D}$. Therefore (see e.g., [13, Lemma 0.13]), $\mathcal{E}$ admits a representation of the form (7.14) in terms of a pair of unitary matrices $U \in \mathbb{C}^{p \times p}$ and $V \in \mathbb{C}^{q \times q}$ which depend only on $\mathcal{R}$ (i.e., only on the interpolation data) and a Schur function $\widehat{\mathcal{E}} \in \mathcal{S}^{(p-\nu) \times(q-\nu)}$, where

$$
\nu=\operatorname{dim} \mathcal{R}_{1}=\operatorname{dim} \mathcal{R}_{2}=\operatorname{dim} \mathcal{R} .
$$

It follows from (6.13) that

$$
\operatorname{dim} \mathcal{R}=\operatorname{rank} \Delta_{\omega}(\omega)-\operatorname{rank} P
$$

By (6.14), rank $\Delta_{\omega}(\omega)$ does not depend on the choice of the point $\omega \in \mathbb{D}$. Setting $\omega=0$ in the latter equality and making use of (6.1) we get

$$
\operatorname{dim} \mathcal{R}=\operatorname{rank} \Delta_{0}(0)-\operatorname{rank} P=\operatorname{rank}\left(M^{*} P M+C_{2}^{*} C_{2}\right)-\operatorname{rank} P,
$$

which together with (7.23) implies (1.11) and completes the proof of theorem.

Note that the inequalities (1.12) are immediate from (7.23) since $\mathcal{R}_{1}$ and $\mathcal{R}_{2}$ are subspaces of $\mathbb{C}^{p}$ and of $\mathbb{C}^{q}$, respectively.

Note also that the description of the set of all solutions of the nondegenerate aBIP (see [16, Theorem 10.1]) can be obtained as a particular case of Theorem 7.4. Indeed, for invertible $P, \nu=0$; therefore, there is no constant block in (7.14) and the parameter $\mathcal{E}$ in (7.13) varies over all of $\mathcal{S}^{p \times q}$. Moreover, the function $\Theta$ is now given by formula (7.6) with $P^{[-1]}$ replaced by $P^{-1}$, which coincides with formula (8.14) from [16].

Proof of Theorem 1.2: Let (1.5) be in force and let $P$ be a nonnegative solution of the Lyapunov-Stein equation (1.8). By Theorem 5.3, there exist matrices $Q, M_{1}$ and $N_{1}$ satisfying conditions (3.22), (5.1), (5.12) and (5.13) and such that $\mathcal{S}(M, N, P, C)=$ $\mathcal{S}\left(M_{1}, N_{1}, P, C\right)$. By Theorem 7.4, the set $\mathcal{S}\left(M_{1}, N_{1}, P, C\right)$ is parametrized by the linear fractional transformation (7.13) based on the $J$-inner function $\Theta$ given by (7.6) with $M$ and $N$ replaced by $M_{1}$ and $N_{1}$, respectively. The parameter $\mathcal{E}$ in (7.13) is of the form

$$
\mathcal{E}(z)=U\left(\begin{array}{cc}
\widehat{\mathcal{E}}(z) & 0 \\
0 & I_{\nu_{1}}
\end{array}\right) V, \quad \widehat{\mathcal{E}} \in \mathcal{S}^{\left(p-\nu_{1}\right) \times\left(q-\nu_{1}\right)}
$$

where $U$ and $V$ are unitary matrices and

$$
\nu_{1}=\operatorname{rank}\left(M_{1}^{*} P M_{1}+C_{2}^{*} C_{2}\right)-\operatorname{rank} P .
$$

In view of $(3.22), \nu_{1}$ is equal to the integer $\nu$ defined via (1.11). Moreover, setting

$$
\Theta(z)=\Theta(z)\left(\begin{array}{cc}
U & 0 \\
0 & V^{*}
\end{array}\right)
$$

it is easily seen that the formulas (7.13) and (1.13) with parameters of the form (7.14) and (1.14), respectively, are equivalent. It remains to note that $\Theta$ is $J$-inner since $\Theta$ is $J$-inner and the matrices $U$ and $V$ are unitary. 


\section{The Redheffer transform}

In the previous section the set $\mathcal{S}(M, N, P, C)$ of all solutions of the $\operatorname{aBIP}(M, N, P, C)$ was parametrized by the linear fractional transformation (7.13) of those $\mathcal{E} \in \mathcal{S}^{p \times q}$ which have the special structure (7.14). It is easily seen from (7.14) that in fact, the independent parameter in (7.13) is the function $\widehat{\mathcal{E}} \in \mathcal{S}^{(p-\nu) \times(q-\nu)}$. In this section we shall parametrize the set $\mathcal{S}(M, N, P, C)$ directly in terms of $\widehat{\mathcal{E}} \in \mathcal{S}^{(p-\nu) \times(q-\nu)}$ via a special Redheffer linear fractional transformation which was already mentioned in Section 1 and which will be explicitly constructed below.

Let $\Theta$ be the $J$-inner mvf given by (7.6). By Corollary 7.3, $\operatorname{det} \theta_{22} \not \equiv 0$ and hence, the $\mathbb{C}^{(p+q) \times(p+q)}$-valued function

$$
\Sigma(z)=\left(\begin{array}{cc}
I_{p} & -\theta_{12}(z) \\
0 & -\theta_{22}(z)
\end{array}\right)^{-1}\left(\begin{array}{cc}
\theta_{11}(z) & 0 \\
\theta_{21}(z) & -I_{q}
\end{array}\right)
$$

is well defined. By (8.1), the entries of $\Sigma$ in the block decomposition

$$
\Sigma=\left(\begin{array}{ll}
\Sigma_{11} & \Sigma_{12} \\
\Sigma_{21} & \Sigma_{22}
\end{array}\right):\left(\begin{array}{l}
\mathbb{C}^{p} \\
\mathbb{C}^{q}
\end{array}\right) \rightarrow\left(\begin{array}{l}
\mathbb{C}^{p} \\
\mathbb{C}^{q}
\end{array}\right)
$$

are given by the formulas

$$
\Sigma_{11}=\theta_{11}-\theta_{12} \theta_{22}^{-1} \theta_{21}, \quad \Sigma_{12}=\theta_{12} \theta_{22}^{-1}, \quad \Sigma_{21}=-\theta_{22}^{-1} \theta_{21}, \quad \Sigma_{22}=\theta_{22}^{-1} .
$$

To obtain the explicit formula for $\Sigma$ in terms of the interpolation data (1.3) we use the function $\Delta_{\mu}^{\dagger}(z)$ defined in $(6.26)$.

Lemma 8.1 Under the assumptions of Theorem 7.4, let $\Theta$ and $\Sigma$ be functions defined by (7.6) and (8.1) respectively. Then

$$
\Sigma(z)=I_{p+q}-\rho_{\mu}(z) J C \Delta_{\mu}^{\dagger}(z) C^{*} J
$$

and

$$
I_{p+q}-\Sigma(z) \Sigma(w)^{*}=\rho_{w}(z) J C \Delta_{\mu}^{\dagger}(z) P \Delta_{\mu}^{\dagger}(w)^{*} C^{*} J .
$$

Moreover, $\Sigma$ is inner in $\mathbb{D}$ and its block entry $\Sigma_{21}$ is strictly contractive in $\overline{\mathrm{D}}$.

Proof: Using (7.3) and the invariance relations (5.14) we represent $\Theta$ in the form (7.9), where the matrices $M_{\diamond}, N_{\diamond}, \widetilde{C}$ and $\widetilde{P}>0$ are subject to the Lyapunov-Stein equation (6.28). It was shown in [16, Theorem 11.2] that for $\Theta$ of the form (7.9) (i.e., with a strictly positive Pick matrix $\widetilde{P}$ ) the associated function $\Sigma$ can be represented as

$$
\Sigma(z)=I_{p+q}-\rho_{\mu}(z) J \widetilde{C} \widetilde{\Delta}_{\mu}^{-1}(z) \widetilde{C}^{*} J
$$

and

$$
I_{p+q}-\Sigma(z) \Sigma(w)^{*}=\rho_{w}(z) J \widetilde{C} \widetilde{\Delta}_{\mu}^{-1}(z) \widetilde{P} \widetilde{\Delta}_{\mu}(w)^{-*} \widetilde{C}^{*} J
$$

where

$$
\widetilde{\Delta}_{\mu}(z):=\left(M_{\diamond}-\mu N_{\diamond}\right)^{*} \widetilde{P}\left(M_{\diamond}-z N_{\diamond}\right)+\rho_{\mu}(z) \widetilde{C}_{2}^{*} \widetilde{C}_{2}
$$


Comparing the latter definition with (6.29) we get

$$
\widetilde{\Delta}_{\mu}(z)=Q^{*} \Delta_{\mu}(z) Q
$$

which, with the help of (6.27) and (6.26), implies (8.4):

$$
\begin{aligned}
\Sigma(z) & =I_{p+q}-\rho_{\mu}(z) J C Q^{*}\left(Q \Delta_{\mu}(z) Q\right)^{-1} Q^{*} C^{*} J \\
& =I_{p+q}-\rho_{\mu}(z) J C \Delta_{\mu}^{\dagger}(z) C^{*} J
\end{aligned}
$$

In much the same way, (8.7) implies (8.5).

By Lemma 6.10, $\Delta_{\mu}^{\dagger}(z)$ is analytic in $\overline{\mathbb{D}}$ and then, (8.5) implies that $\Sigma$ is inner in $\mathbb{D}$. Finally, since $\Sigma_{21}=-\theta_{22}^{-1} \theta_{21}$, the last assertion of the lemma is immediate from Corollary 7.3.

The next theorem expresses the set of all solutions of the aBIP in terms of the Redheffer transform of a parameter which is still of special structure.

Theorem 8.2 Under assumptions of Theorem 7.4, let $\Sigma_{j k}$ be the block entries in the decomposition (8.2) of the mvf $\Theta$ defined in (8.4). Then all the solutions $S$ of the $\operatorname{aBIP}(M, N, P, C)$ are parametrized by the transformation

$$
S(z)=\Sigma_{12}(z)+\Sigma_{11}(z) \mathcal{E}(z)\left(I_{q}-\Sigma_{21}(z) \mathcal{E}(z)\right)^{-1} \Sigma_{22}(z)
$$

in which the parameter $\mathcal{E} \in \mathcal{S}^{p \times q}$ is of the form (7.14).

Proof: It suffices to note that (7.13) can be written in the form (8.8) for the $\Sigma_{j k}$ expressed in terms of the $\theta_{j k}$ by (8.3). The latter equalities display the link (8.1) between $\Sigma$ and $\Theta$ and thus, $\Sigma$ is of the form (8.4), by Lemma 8.1 .

The transformation (8.8) is called the Redheffer transform based on the transfer function $\Sigma$ of the parameter $\mathcal{E}$. For future purposes it is convenient to recall some well known facts for Redheffer transforms based on general mvf's $\Sigma$ in the Schur class $\mathcal{S}^{(p+q) \times(p+q)}$ which are partitioned conformally with $J$ (see e.g., [25]).

Lemma 8.3 Let $\Sigma$ be partitioned as in (8.2) and let $S$ be of the form (8.8). Then

$$
\begin{aligned}
I-S S^{*}= & \Sigma_{11}\left(I-\mathcal{E} \Sigma_{21}\right)^{-1}\left(I-\mathcal{E} \mathcal{E}^{*}\right)\left(I-\mathcal{E} \Sigma_{21}\right)^{-*} \Sigma_{11}^{*} \\
& +\left(I, \Sigma_{11} \mathcal{E}\left(I-\Sigma_{21} \mathcal{E}\right)^{-1}\right)\left(I-\Sigma \Sigma^{*}\right)\left(\begin{array}{c}
I \\
\left(I-\mathcal{E}^{*} \Sigma_{21}^{*}\right)^{-1} \mathcal{E}^{*} \Sigma_{11}^{*}
\end{array}\right),
\end{aligned}
$$

whenever $\left(I-\mathcal{E} \Sigma_{21}\right)^{-1}$ exists.

Proof: Using (8.3), (8.8) and the identity

$$
I+\mathcal{E}\left(I-\Sigma_{21} \mathcal{E}\right)^{-1} \Sigma_{21}=\left(I-\mathcal{E} \Sigma_{21}\right)^{-1}
$$

we obtain the formula

$$
\left(I, \Sigma_{11} \mathcal{E}\left(I-\Sigma_{21} \mathcal{E}\right)^{-1}\right) \Sigma=\left(\Sigma_{11}\left(I-\mathcal{E} \Sigma_{21}\right)^{-1}, S\right)
$$


Therefore, since $\mathcal{E}\left(I-\Sigma_{21} \mathcal{E}\right)^{-1}=\left(I-\mathcal{E} \Sigma_{21}\right)^{-1} \mathcal{E}$, it follows that

$$
\begin{aligned}
\left(I, \Sigma_{11} \mathcal{E}\left(I-\Sigma_{21} \mathcal{E}\right)^{-1}\right)\left(I-\Sigma \Sigma^{*}\right)\left(\begin{array}{c}
I \\
\left(I-\mathcal{E}^{*} \Sigma_{21}^{*}\right)^{-1} \mathcal{E}^{*} \Sigma_{11}^{*}
\end{array}\right) \\
=I+\Sigma_{11} \mathcal{E}\left(I-\Sigma_{21} \mathcal{E}\right)^{-1}\left(I-\mathcal{E}^{*} \Sigma_{21}^{*}\right)^{-1} \mathcal{E}^{*} \Sigma_{11}^{*} \\
\quad-\Sigma_{11}\left(I-\mathcal{E} \Sigma_{21}\right)^{-1}\left(I-\Sigma_{21}^{*} \mathcal{E}^{*}\right)^{-1} \Sigma_{11}^{*}-S S^{*} \\
=I-S S^{*}-\Sigma_{11}\left(I-\mathcal{E} \Sigma_{21}\right)^{-1}\left(I-\mathcal{E} \mathcal{E}^{*}\right)\left(I-\mathcal{E} \Sigma_{21}\right)^{-*} \Sigma_{11}^{*}
\end{aligned}
$$

which is equivalent to $(8.9)$.

Corollary 8.4 If $\Sigma$ and $\mathcal{E}$ are inner in $\mathbb{D}$, then $S$ is inner too. If $\Sigma$ is strictly contractive in $\overline{\mathrm{D}}$, then $S$ is also strictly contractive in $\overline{\mathbb{D}}$.

Proof: If $\Sigma$ and $\mathcal{E}$ are inner in $\mathbb{D}$, then both of the terms on the right hand side of (8.9) are nonnegative inside $\mathbb{D}$ and are equal to zero almost everywhere on the unit circle. This proves the first assertion. If $\Sigma$ is strictly contractive in $\overline{\mathrm{D}}$, then the second term on the right hand side of (8.9) is strictly positive in $\overline{\mathbb{D}}$ which means that $S$ is strictly contractive in $\overline{\mathbb{D}}$.

The next theorem gives a new description of the set of solutions of the aBIP in terms of a new Redheffer transformation which is based on a $(p+q-\nu) \times(p+q-\nu)$ matrix valued inner function and in which the parameter is now an arbitrary element of $\mathcal{S}^{(p-\nu) \times(q-\nu)}$. It is included primarily for the sake of comparison with the representation which will be established in Theorem 9.4 below, by other methods.

Theorem 8.5 Let $U \in \mathbb{C}^{p \times p}$ and $V \in \mathbb{C}^{q \times q}$ be unitary matrices from the representation (7.14) depending only on the data (1.3), let $\Sigma_{j k}$ be the block entries in the block decomposition (8.2) of the function $\Sigma$ given by (8.1), let $\nu$ be the integer defined via (1.11) and let $\gamma_{11} \in$ $\mathcal{S}^{(q-\nu) \times(p-\nu)}, \gamma_{12} \in \mathcal{S}^{(q-\nu) \times \nu}, \gamma_{21} \in \mathcal{S}^{\nu \times(p-\nu)}, \gamma_{22} \in \mathcal{S}^{\nu \times \nu}$ be the functions defined as

$$
\left(\begin{array}{cc}
\gamma_{11}(z) & \gamma_{12}(z) \\
\gamma_{21}(z) & \gamma_{22}(z)
\end{array}\right)=V \Sigma_{21}(z) U=\rho_{\mu}(z) V C_{2} \Delta_{\mu}^{\dagger}(z) C_{1}^{*} U
$$

Then all the solutions $S$ of the $\mathbf{a B I P}(M, N, P, C)$ are parametrized by the Redheffer transform

$$
S(z)=\Psi_{12}(z)+\Psi_{11}(z) \widehat{\mathcal{E}}(z)\left(I_{q-\nu}-\Psi_{21}(z) \widehat{\mathcal{E}}(z)\right)^{-1} \Psi_{22}(z),
$$

where

$$
\begin{aligned}
& \Psi_{11}(z)=\Sigma_{11}(z) U\left(\begin{array}{c}
I_{p-\nu} \\
\left(I_{\nu}-\gamma_{22}(z)\right)^{-1} \gamma_{21}(z)
\end{array}\right) \\
& \Psi_{12}(z)=\Sigma_{12}(z)+\Sigma_{11}(z) U\left(\begin{array}{cc}
0 & 0 \\
0 & \left(I_{\nu}-\gamma_{22}(z)\right)^{-1}
\end{array}\right) V \Sigma_{22}(z), \\
& \Psi_{21}(z)=\gamma_{11}(z)+\gamma_{12}(z)\left(I_{\nu}-\gamma_{22}(z)\right)^{-1} \gamma_{21}(z) \\
& \Psi_{22}(z)=\left(I_{q-\nu}, \gamma_{12}(z)\left(I_{\nu}-\gamma_{22}(z)\right)^{-1}\right) V \Sigma_{22}(z)
\end{aligned}
$$


and $\widehat{\mathcal{E}}$ is an independent free parameter from $\mathcal{S}^{(p-\nu) \times(q-\nu)}$. The function

$$
\Psi=\left(\begin{array}{ll}
\Psi_{11} & \Psi_{12} \\
\Psi_{21} & \Psi_{22}
\end{array}\right)
$$

is inner in $\mathbb{D}$ and the block $\Psi_{21}$ is strictly contractive in $\overline{\mathrm{D}}$.

Proof: By Theorem 7.4, all the solutions $S$ of the $\operatorname{aBIP}(M, N, P, C)$ are parametrized by the formula (8.8) (which is equivalent to (7.13)) when $\mathcal{E}$ varies over $\mathcal{S}^{p \times q}$ and is of the form (7.14). We rewrite (8.8) as

$$
S(z)=\Sigma_{12}(z)+\Sigma_{11}(z) U\left(\begin{array}{cc}
\widehat{\mathcal{E}}(z) & 0 \\
0 & I_{\nu}
\end{array}\right)\left(I_{q}-V \Sigma_{21}(z) U\left(\begin{array}{cc}
\widehat{\mathcal{E}}(z) & 0 \\
0 & I_{\nu}
\end{array}\right)\right)^{-1} V \Sigma_{22}(z) .
$$

Using the block decomposition (8.10) of $V \Sigma_{21} U$ and an inversion formula based on Schur complements (see e.g., [13, Section 0]), we get

$$
\begin{gathered}
\left(I_{q}-V \Sigma_{21} U\left(\begin{array}{cc}
\widehat{\mathcal{E}} & 0 \\
0 & I_{\nu}
\end{array}\right)\right)^{-1}=\left(\begin{array}{cc}
I_{q-\nu}-\gamma_{11} \widehat{\mathcal{E}} & -\gamma_{12} \\
-\gamma_{21} & I_{\nu}-\gamma_{22}
\end{array}\right)^{-1} \\
=\left(\begin{array}{cc}
0 & 0 \\
0 & \left(I_{\nu}-\gamma_{22}\right)^{-1}
\end{array}\right)+\left(\begin{array}{c}
I_{q-\nu} \\
\left(I_{\nu}-\gamma_{22}\right)^{-1} \gamma_{21} \widehat{\mathcal{E}}
\end{array}\right)\left(\begin{array}{c}
\left.I_{q-\nu}-\left(\gamma_{11}+\gamma_{12}\left(I_{\nu}-\gamma_{22}\right)^{-1} \gamma_{21}\right) \widehat{\mathcal{E}}\right)^{-1} \\
\times\left(I_{q-\nu}, \gamma_{12}\left(I_{\nu}-\gamma_{22}\right)^{-1}\right) .
\end{array}\right.
\end{gathered}
$$

Therefore, in view of (8.14),

$$
\begin{aligned}
& \left(\begin{array}{cc}
\widehat{\mathcal{E}} & 0 \\
0 & I_{\nu}
\end{array}\right)\left(I_{q}-V \Sigma_{21} U\left(\begin{array}{cc}
\widehat{\mathcal{E}} & 0 \\
0 & I_{\nu}
\end{array}\right)\right)^{-1} \\
& =\left(\begin{array}{cc}
0 & 0 \\
0 & \left(I_{\nu}-\gamma_{22}\right)^{-1}
\end{array}\right)+\left(\begin{array}{c}
I_{p-\nu} \\
\left(I_{\nu}-\gamma_{22}\right)^{-1} \gamma_{21}
\end{array}\right) \widehat{\mathcal{E}}\left(I_{q-\nu}-\Psi_{21} \widehat{\mathcal{E}}\right)^{-1}\left(I_{q-\nu}, \gamma_{12}\left(I_{\nu}-\gamma_{22}\right)^{-1}\right) .
\end{aligned}
$$

Substituting the last equality into (8.17) and taking into account (8.12), (8.13) and (8.15), we obtain (8.11).

By (8.10) and (8.14), $\Psi_{21}$ is the Redheffer transformation based on the transfer function $U \Sigma_{21}(z) V$, of the inner parameter $\widehat{\mathcal{E}}=I_{\nu}$. By Corollary $7.3, V \Sigma_{21}(z) U$ is strictly contractive in $\overline{\mathrm{D}}$ and therefore, $\Psi_{21}$ is strictly contractive in $\overline{\mathrm{D}}$ by Corollary 8.4.

Using the block decompositions

$$
\Sigma_{11} U=\left(\alpha_{1}, \alpha_{2}\right) \quad \text { and } \quad V \Sigma_{22}=\left(\begin{array}{c}
\delta_{1} \\
\delta_{2}
\end{array}\right) \quad\left(\alpha_{2} \in \mathcal{S}^{p \times \nu}, \quad \delta_{2} \in \mathcal{S}^{\nu \times q}\right),
$$

we rewrite formulas (8.12), (8.13) and (8.15) as

$$
\begin{aligned}
& \Psi_{11}=\alpha_{1}+\alpha_{2}\left(I_{\nu}-\gamma_{22}\right)^{-1} \gamma_{21} \\
& \Psi_{12}=\Sigma_{12}+\alpha_{2}\left(I_{\nu}-\gamma_{22}\right)^{-1} \delta_{2} \\
& \Psi_{22}=\delta_{1}+\gamma_{12}\left(I_{\nu}-\gamma_{22}\right)^{-1} \delta_{2}
\end{aligned}
$$


and substitute them together with (8.14) into (8.16):

$$
\Psi=\left(\begin{array}{ll}
\Psi_{11} & \Psi_{12} \\
\Psi_{21} & \Psi_{22}
\end{array}\right)=\left(\begin{array}{cc}
\alpha_{1} & \Sigma_{12} \\
\gamma_{11} & \delta_{1}
\end{array}\right)+\left(\begin{array}{c}
\alpha_{2} \\
\gamma_{12}
\end{array}\right)\left(I_{\nu}-\gamma_{22}\right)^{-1}\left(\gamma_{21}, \delta_{2}\right) .
$$

Thus, $\Psi$ is the Redheffer transformation based on the transfer function

$$
\boldsymbol{\Sigma}=\left(\begin{array}{c|cc}
\alpha_{2} & \alpha_{1} & \Sigma_{12} \\
\gamma_{12} & \gamma_{11} & \delta_{1} \\
-- & -- & -- \\
\gamma_{22} & \gamma_{21} & \delta_{2}
\end{array}\right)
$$

of the inner parameter $\widehat{\mathcal{E}} \equiv I_{\nu}$. Comparing the latter decomposition of $\boldsymbol{\Sigma}$ with (8.2) and taking into account (8.10) and (8.18), we conclude that

$$
\Sigma(z)=\left(\begin{array}{cc}
I_{p} & 0 \\
0 & V
\end{array}\right) \Sigma(z)\left(\begin{array}{cc}
U & 0 \\
0 & I_{q}
\end{array}\right)\left(\begin{array}{ccc}
0 & I_{\nu} & 0 \\
I_{p-\nu} & 0 & 0 \\
0 & 0 & I_{q}
\end{array}\right) .
$$

By Corollary $7.3, \Sigma$ is inner in $\mathbb{D}$ and therefore, $\Sigma$ is also inner. Thus, by Corollary 8.4, $\Psi$ is inner in $\mathbb{D}$.

We remark that all three of the representations (7.13), (8.8) and (8.11) are based on normalizations which are imposed at a point $\mu \in \mathbb{\Gamma}$ : by (7.6) and (8.6), $\Theta(\mu)=\Sigma(\mu)=I_{p+q}$ and, as follows from (8.12)-(8.15),

$$
\Psi(\mu)=\left(\begin{array}{cc}
U & 0 \\
0 & I_{q-\nu}
\end{array}\right)\left(\begin{array}{ccc}
I_{p-\nu} & 0 & 0 \\
0 & 0 & I_{\nu} \\
0 & I_{q-\nu} & 0
\end{array}\right)\left(\begin{array}{cc}
I_{p-\nu} & 0 \\
0 & V
\end{array}\right) .
$$

In the next section we shall present a parametrization of the set of all solutions to the aBIP in terms of a Redheffer transform which is based on a $(p+q-\nu) \times(p+q-\nu)$ matrix valued inner function which is normalized at a point $\omega \in \mathbb{D}$.

\section{Another approach}

In this section we establish a Redheffer type representation for the set of solutions to the aBIP based on the methods of [23]. The explicit formulas for the transfer function $\Psi$ will be given in Section 10. This extends the analysis which was carried out in [16] which allowed singular $P$ but required $\Delta_{\omega}(\omega)$ to be invertible. Here we relax this requirement and assume that $\operatorname{rank} \Delta_{\omega}(\omega)=n-k$.

As in [16], the function $\Delta_{\omega}(z)(\omega \in \mathbb{D})$ plays a central role in the present analysis. Let

$$
W_{1}=\left(\begin{array}{c}
P^{\frac{1}{2}} G(\omega) \\
\rho_{\omega}(\omega)^{\frac{1}{2}} C_{2}
\end{array}\right) \quad \text { and } \quad W_{2}=\left(\begin{array}{c}
-P^{\frac{1}{2}} H(\omega)^{*} \\
\rho_{\omega}(\omega)^{\frac{1}{2}} C_{1}
\end{array}\right)
$$

Evaluating (6.1) and (6.2) at the point $\omega$ we get

$$
\Delta_{\omega}(\omega)=G(\omega)^{*} P G(\omega)+\rho_{\omega}(\omega) C_{2}^{*} C_{2}=H(\omega) P H(\omega)^{*}+\rho_{\omega}(\omega) C_{1}^{*} C_{1},
$$


which can be written as

$$
\Delta_{\omega}(\omega)=W_{1}^{*} W_{1}=W_{2}^{*} W_{2}
$$

and guarantees that the linear map

$$
\mathrm{V}: W_{1} x \longrightarrow W_{2} x
$$

is an isometry from $\mathcal{D}_{\mathbf{V}}=\operatorname{Ran} W_{1} \subset \mathbb{C}^{n+q}$ onto $\mathcal{R}_{\mathbf{V}}=\operatorname{Ran} W_{2} \subset \mathbb{C}^{n+p}$. By (9.3),

$$
\operatorname{dim} \mathcal{D}_{\mathbf{V}}=\operatorname{dim} \mathcal{R}_{\mathbf{V}}=\operatorname{rank} \Delta_{\omega}(\omega)=n-k .
$$

Thus, the dimensions of the orthogonal complements

$$
\mathcal{D}_{\mathbf{V}}^{\perp}=\mathbb{C}^{n+q} \ominus \mathcal{D}_{\mathbf{V}} \quad \text { and } \quad \mathcal{R}_{\mathbf{V}}^{\perp}=\mathbb{C}^{n+p} \ominus \mathcal{R}_{\mathbf{V}}
$$

are equal to

$$
q^{\prime}:=\operatorname{dim} \mathcal{D}_{\mathbf{V}}^{\perp}=k+q \quad \text { and } \quad p^{\prime}:=\operatorname{dim} \mathcal{R}_{\mathbf{V}}^{\perp}=k+p,
$$

respectively.

Remark 9.1 The orthogonal projection $\mathbf{P}_{\mathcal{D}_{\mathbf{V}}^{\perp}}$ of $\mathbb{C}^{n+q}$ onto $\mathcal{D}_{\mathbf{V}}^{\perp}$ is given by the formula

$$
\mathbf{P}_{\mathcal{D}_{\mathbf{V}}^{\perp}}=I_{n+q}-W_{1}\left(W_{1}^{*} W_{1}\right)^{[-1]} W_{1}^{*}=I_{n+q}-W_{1} \Delta_{\omega}^{[-1]}(\omega) W_{1}^{*},
$$

whereas the orthogonal projection $\mathbf{P}_{\mathcal{R}_{\mathbf{V}}^{\perp}}$ of $\mathbb{C}^{n+p}$ onto $\mathcal{R}_{\mathbf{V}}^{\perp}$ is given by the formula

$$
\mathbf{P}_{\mathcal{R}_{\mathbf{V}}^{\perp}}=I_{n+p}-W_{2}\left(W_{2}^{*} W_{2}\right)^{[-1]} W_{2}^{*}=I_{n+p}-W_{2} \Delta_{\omega}^{[-1]}(\omega) W_{2}^{*} .
$$

Let $W_{1}^{\perp} \in \mathbb{C}^{(n+q) \times q^{\prime}}$ and $W_{2}^{\perp} \in \mathbb{C}^{(n+p) \times p^{\prime}}$ be isometric matrices whose columns span $\mathcal{D}_{\mathbf{V}}^{\perp}$ and $\mathcal{R}_{\mathbf{V}}^{\perp}$ respectively. Then the projections $\mathbf{P}_{\mathcal{D}_{\mathbf{V}}^{\perp}}$ and $\mathbf{P}_{\mathcal{R}_{\mathbf{V}}^{\perp}}$ can also be expressed as

$$
\mathbf{P}_{\mathcal{D}_{\mathbf{v}}^{\perp}}=W_{1}^{\perp}\left(W_{1}^{\perp}\right)^{*} \quad \text { and } \quad \mathbf{P}_{\mathcal{R}_{\mathbf{v}}^{\perp}}=W_{2}^{\perp}\left(W_{2}^{\perp}\right)^{*}
$$

respectively. Moreover, the formulas

$$
\left(W_{1}^{\perp}\right)^{*} W_{1}^{\perp}=I_{q^{\prime}}, \quad\left(W_{2}^{\perp}\right)^{*} W_{2}^{\perp}=I_{p^{\prime}}, \quad\left(W_{1}^{\perp}\right)^{*} W_{1}=0 \quad \text { and } \quad\left(W_{2}^{\perp}\right)^{*} W_{2}=0
$$

hold by definition.

Lemma 9.2 The operator

$$
\mathbf{U}=\left(\begin{array}{ccc}
U_{11} & U_{12} & U_{13} \\
U_{21} & U_{22} & U_{23} \\
U_{31} & U_{32} & 0
\end{array}\right): \quad\left(\begin{array}{l}
\mathbb{C}^{n} \\
\mathbb{C}^{q} \\
\mathbb{C}^{p^{\prime}}
\end{array}\right) \rightarrow\left(\begin{array}{l}
\mathbb{C}^{n} \\
\mathbb{C}^{p} \\
\mathbb{C}^{q^{\prime}}
\end{array}\right)
$$

with entries specified by the rules

$$
\begin{aligned}
\left(\begin{array}{cc}
U_{11} & U_{12} \\
U_{21} & U_{22}
\end{array}\right) & =\left(\begin{array}{c}
-P^{\frac{1}{2}} H(\omega)^{*} \\
\rho_{\omega}(\omega)^{\frac{1}{2}} C_{1}
\end{array}\right) \Delta_{\omega}^{[-1]}(\omega)\left(G(\omega)^{*} P^{\frac{1}{2}}, \rho_{\omega}(\omega)^{\frac{1}{2}} C_{2}^{*}\right) \\
& =W_{2} \Delta_{\omega}^{[-1]}(\omega) W_{1}^{*} \\
\left(U_{31}, U_{32}\right) & =\left(W_{1}^{\perp}\right)^{*} \quad \text { and } \quad\left(\begin{array}{c}
U_{13} \\
U_{23}
\end{array}\right)=W_{2}^{\perp}
\end{aligned}
$$

is a unitary extension of the isometry $\mathbf{V}$ defined by (9.4). 
Proof: It follows from (9.3) and (9.8) that

$$
\begin{aligned}
\left(\begin{array}{cc}
U_{11} & U_{12} \\
U_{21} & U_{22}
\end{array}\right)^{*}\left(\begin{array}{ll}
U_{11} & U_{12} \\
U_{21} & U_{22}
\end{array}\right) & =W_{1} \Delta_{\omega}^{[-1]}(\omega) W_{2}^{*} W_{2} \Delta_{\omega}^{[-1]}(\omega) W_{2}^{*} \\
& =W_{1} \Delta_{\omega}^{[-1]}(\omega) W_{1}^{*}
\end{aligned}
$$

which together with (9.5), (9.6) and (9.9) implies that

$$
\left(\begin{array}{ll}
U_{11} & U_{12} \\
U_{21} & U_{22}
\end{array}\right)^{*}\left(\begin{array}{ll}
U_{11} & U_{12} \\
U_{21} & U_{22}
\end{array}\right)+\left(\begin{array}{c}
U_{31}^{*} \\
U_{32}^{*}
\end{array}\right)\left(U_{31}, U_{32}\right)=I_{n+q}
$$

Continuing in the same vein, it is readily checked that $\mathbf{U}$ is an isometry. Since $\mathbf{U}$ acts from the finite dimensional space $\mathbb{C}^{n+p+q^{\prime}}$ into itself, it is unitary.

To show that $\mathbf{U}$ is an extension of $\mathbf{V}$, it suffices to check that

$$
\left(\begin{array}{ll}
U_{11} & U_{12} \\
U_{21} & U_{22}
\end{array}\right) W_{1} u=W_{2} u \quad\left(\forall u \in \mathbb{C}^{n}\right) .
$$

Let $\mathrm{Q} \in \mathbb{C}^{n \times(n-k)}$ be the isometric matrix from (6.19). Then every vector $u \in \mathbb{C}^{n}$ can be represented in the form $u=y+\mathbf{Q} x$ as in (6.25). By (9.3),

$$
W_{1} y=0 \quad \text { and } \quad W_{2} y=0 \quad(\forall y \in \mathcal{K}) .
$$

Therefore,

$$
\begin{aligned}
\left(\begin{array}{ll}
U_{11} & U_{12} \\
U_{21} & U_{22}
\end{array}\right) W_{1} u & =W_{2} \Delta_{\omega}^{[-1]}(\omega) W_{1}^{*} W_{1} u \\
& =W_{2} \mathbf{Q}\left(\mathbf{Q}^{*} \Delta_{\omega}(\omega) \mathbf{Q}\right)^{-1} \mathbf{Q}^{*} \Delta_{\omega}(\omega)(\mathbf{Q} x+y) \\
& =W_{2} \mathbf{Q} x=W_{2}(\mathbf{Q} x+y)=W_{2} u
\end{aligned}
$$

which ends the proof of lemma.

The next step is to define the function

$$
\begin{aligned}
\widetilde{\Sigma}(z) & =\left(\begin{array}{cc}
\widetilde{\Sigma}_{11}(z) & \widetilde{\Sigma}_{12}(z) \\
\widetilde{\Sigma}_{21}(z) & \widetilde{\Sigma}_{22}(z)
\end{array}\right) \\
& =\left(\begin{array}{cc}
U_{23} & U_{22} \\
0 & U_{32}
\end{array}\right)+z\left(\begin{array}{c}
U_{21} \\
U_{31}
\end{array}\right)\left(I-z U_{11}\right)^{-1}\left(U_{13}, U_{12}\right)
\end{aligned}
$$

Let us consider the auxiliary $\operatorname{aBIP}\left(G(\omega), H(\omega)^{*}, P, \rho_{\omega}(\omega)^{\frac{1}{2}} C\right)$ : find all the functions $\widetilde{S} \in$ $\mathcal{S}^{p \times q}$ such that

$$
\left(\begin{array}{cc}
I_{p} & -\widetilde{S}(\zeta) \\
-\widetilde{S}(\zeta)^{*} & I_{q}
\end{array}\right) \widetilde{C} \widetilde{G}(\zeta)^{-1} \in\left(\begin{array}{c}
\mathbf{H}_{2}^{p \times n} \\
\left(\mathbf{H}_{2}^{q \times n}\right)^{\perp}
\end{array}\right)
$$

and

$$
\left\langle\left(\begin{array}{cc}
I_{p} & -\widetilde{S}(\zeta) \\
-\widetilde{S}(\zeta)^{*} & I_{q}
\end{array}\right) \widetilde{C} \widetilde{G}(\zeta)^{-1} y, \widetilde{C} \widetilde{G}(\zeta)^{-1} x\right\rangle_{L_{2}^{p+q}(\mathbb{T})}=x^{*} P y \quad\left(\forall x, y \in \mathbb{C}^{n}\right) .
$$


where

$$
\widetilde{G}(\zeta)=G(\omega)+\zeta H(\omega)^{*}=M-\omega N+\zeta(\bar{\omega} M-N) \quad \text { and } \quad \widetilde{C}=\rho_{\omega}(\omega)^{\frac{1}{2}} C .
$$

This problem is well posed because $\widetilde{G}(\zeta)$ is invertible on $\mathbb{T}$. Moreover, it admits a solution, since $P$ is a nonnegative solution of the requisite Lyapunov-Stein equation, as follows easily from (9.2). By the general result from [23], all solutions $S$ of the $\operatorname{aBIP}\left(G(\omega), H(\omega)^{*}, P, \rho_{\omega}(\omega)^{\frac{1}{2}} C\right)$ are parametrized by the formula

$$
\widetilde{S}(z)=\widetilde{\Sigma}_{12}(z)+\widetilde{\Sigma}_{11}(z) \widetilde{\mathcal{E}}(z)\left(I_{q}-\widetilde{\Sigma}_{21}(z) \widetilde{\mathcal{E}}(z)\right)^{-1} \widetilde{\Sigma}_{22}(z)
$$

when $\widetilde{\mathcal{E}}$ is the parameter varying over the set $\mathcal{S}^{q^{\prime} \times p^{\prime}}$ of $\mathbb{C}^{q^{\prime} \times p^{\prime}}$-valued Schur functions.

Lemma 9.3 A function $\widetilde{S}$ is a solution of the $\operatorname{aBIP}\left(G(\omega), H(\omega)^{*}, P, \rho_{\omega}(\omega)^{\frac{1}{2}} C\right)$ if and only if the function

$$
S(z):=\widetilde{S}\left(\frac{\delta_{\omega}(z)}{\rho_{\omega}(z)}\right)
$$

is a solution of the initial $\mathbf{a B I P}(M, N, P, C)$.

Proof: It follows from (9.12) that

$$
\widetilde{C} \widetilde{G}^{-1}\left(\frac{\delta_{\omega}(z)}{\rho_{\omega}(z)}\right)=\frac{\rho_{\omega}(z)}{\rho_{\omega}(\omega)^{\frac{1}{2}}} C(M-z N)^{-1}=\frac{\rho_{\omega}(z)}{\rho_{\omega}(\omega)^{\frac{1}{2}}} C G(z)^{-1} .
$$

Note also that for every $X$ and $Y$,

$$
X(\zeta) \in \mathbf{H}_{2} \Leftrightarrow \frac{1}{\rho_{\omega}(z)} X\left(\frac{\delta_{\omega}(z)}{\rho_{\omega}(z)}\right) \in \mathbf{H}_{2}
$$

and

$$
Y(\zeta) \in\left(\mathbf{H}_{2}\right)^{\perp} \Leftrightarrow \frac{1}{\rho_{\omega}(z)} Y\left(\frac{\delta_{\omega}(z)}{\rho_{\omega}(z)}\right) \in\left(\mathbf{H}_{2}\right)^{\perp} .
$$

Applying this latter remark to

$$
X(\zeta)=\left(\begin{array}{ll}
I_{p} & -\widetilde{S}(\zeta)
\end{array}\right) \widetilde{C} \widetilde{G}(\zeta)^{-1}, \quad Y(\zeta)=\left(\begin{array}{ll}
-\widetilde{S}(\zeta)^{*} & I_{q}
\end{array}\right) \widetilde{C} \widetilde{G}(\zeta)^{-1}
$$

and taking into account (9.14) and (9.15), we get

$$
\frac{1}{\rho_{\omega}(\omega)^{\frac{1}{2}}}\left(I_{p}-S(\zeta)\right) C G(\zeta)^{-1} \in \mathbf{H}_{2}^{p \times n}, \quad \frac{1}{\rho_{\omega}(\omega)^{\frac{1}{2}}}\left(-S(\zeta)^{*} \quad I_{q}\right) C G(\zeta)^{-1} \in\left(\mathbf{H}_{2}^{q \times n}\right)^{\perp}
$$

which are equivalent to (1.6). Finally, upon representing the expression on the left hand side of (9.11) as an integral, changing variables

$$
\zeta=e^{i t}=\frac{e^{i \tau}-\omega}{1-e^{i \tau} \bar{\omega}}, \quad d t=\frac{\rho_{\omega}(\omega)}{\left|\rho_{\omega}\left(e^{i \tau}\right)\right|^{2}} d \tau
$$


and using (9.14) and (9.15) we obtain

$$
\begin{aligned}
& \left\langle\left(\begin{array}{cc}
I_{p} & -\widetilde{S}(\zeta) \\
-\widetilde{S}(\zeta)^{*} & I_{q}
\end{array}\right) \widetilde{C} \widetilde{G}(\zeta)^{-1} y, \widetilde{C} \widetilde{G}(\zeta)^{-1} x\right\rangle_{L_{2}^{p+q}(\mathbb{T})} \\
& =\frac{1}{2 \pi} \int_{0}^{2 \pi} x^{*} \widetilde{G}\left(e^{i t}\right)^{-*} \widetilde{C}^{*}\left(\begin{array}{cc}
I_{p} & -\widetilde{S}\left(e^{i t}\right) \\
-\widetilde{S}\left(e^{i t}\right)^{*} & I_{q}
\end{array}\right) \widetilde{C} \widetilde{G}\left(e^{i t}\right)^{-1} y d t \\
& =\frac{1}{2 \pi} \int_{0}^{2 \pi} x^{*} G\left(e^{i \tau}\right)^{-*} C^{*}\left(\begin{array}{cc}
I_{p} & -S\left(e^{i \tau}\right) \\
-S\left(e^{i \tau}\right)^{*} & I_{q}
\end{array}\right) C G\left(e^{i \tau}\right)^{-1} y d \tau \\
& =\left\langle\left(\begin{array}{cc}
I_{p} & -S(\zeta) \\
-S(\zeta)^{*} & I_{q}
\end{array}\right) C G(\zeta)^{-1} y, C G(\zeta)^{-1} x\right\rangle_{L_{2}^{p+q}(\mathbb{T})},
\end{aligned}
$$

which ends the proof of lemma.

As a corollary we obtain the following result:

Theorem 9.4 Let $\widetilde{\Sigma}$ be the function defined in (9.10) and let

$$
\Sigma(z)=\left(\begin{array}{cc}
\Sigma_{11}(z) & \Sigma_{12}(z) \\
\Sigma_{21}(z) & \Sigma_{22}(z)
\end{array}\right):=\widetilde{\Sigma}\left(\frac{\delta_{\omega}(z)}{\rho_{\omega}(z)}\right)
$$

Then all the solutions $S$ of the $\mathbf{a B I P}(M, N, P, C)$ are parametrized by the formula

$$
S(z)=\Sigma_{12}(z)+\Sigma_{11}(z) \mathcal{E}(z)\left(I_{q}-\Sigma_{21}(z) \mathcal{E}(z)\right)^{-1} \Sigma_{22}(z)
$$

when the parameter $\mathcal{E}$ varies over the Schur class $\mathcal{S}^{q^{\prime} \times p^{\prime}}$.

Proof: By Lemma 9.3, $S$ is a solution of the $\operatorname{aBIP}(M, N, P, C)$ if and only if it is of the form (9.14) for some solution $\widetilde{S}$ of the auxiliary $\operatorname{aBIP}\left(G(\omega), H(\omega)^{*}, P, \rho_{\omega}(\omega)^{\frac{1}{2}} C\right)$. But each such solution admits a representation of the form (9.13) for some choice of the parameter $\widetilde{\mathcal{E}} \in \mathcal{S}^{q^{\prime} \times p^{\prime}}$. Replacing $z$ by $\frac{\delta_{\omega}(z)}{\rho_{\omega}(z)}$ in (9.13) and taking (9.14) and (9.16) into account, we conclude that $S$ admits a representation of the form $(9.17)$ with $\mathcal{E}(z)=\widetilde{\mathcal{E}}\left(\frac{\delta_{\omega}(z)}{\rho_{\omega}(z)}\right)$. It remains to note that $\mathcal{E}$ runs over all of $\mathcal{S}^{q^{\prime} \times p^{\prime}}$ when $\widetilde{\mathcal{E}}$ does.

\section{Explicit formulas}

In this section we calculate the entries in the inner mvf

$$
\left(\begin{array}{cc}
\Sigma_{11}(z) & \Sigma_{12}(z) \\
\Sigma_{21}(z) & \Sigma_{22}(z)
\end{array}\right)=\left(\begin{array}{cc}
U_{23} & U_{22} \\
0 & U_{32}
\end{array}\right)+\delta_{\omega}(z)\left(\begin{array}{c}
U_{21} \\
U_{31}
\end{array}\right)\left(\rho_{\omega}(z) I-\delta_{\omega}(z) U_{11}\right)^{-1}\left(U_{13}, U_{12}\right)
$$

which is based on the unitary operator $\mathbf{U}$ described in Lemma 9.2. The formulas and calculations are much the same as those which are given in Section 12.5 of [16] except that 
here we have dropped the assumption that $\Delta_{\omega}(\omega)>0$. Consequently, the term $\Delta_{\omega}^{[-1]}(z)$ now appears in place of $\Delta_{\omega}^{-1}(z)$ in the corresponding formulas in [16]. The following identities

$$
\begin{aligned}
& \frac{I_{p+q^{\prime}}-\Sigma(z) \Sigma(\lambda)^{*}}{\rho_{\lambda}(z) \rho_{\omega}(\omega)}=\left(\begin{array}{c}
U_{21} \\
U_{31}
\end{array}\right)\left(\rho_{\omega}(z) I-\delta_{\omega}(z) U_{11}\right)^{-1}\left(\rho_{\omega}(\lambda) I-\delta_{\omega}(\lambda) U_{11}\right)^{-*}\left(U_{21}^{*}, U_{31}^{*}\right) \\
& \frac{I_{q+p^{\prime}}-\Sigma(\lambda)^{*} \Sigma(z)}{\rho_{\lambda}(z) \rho_{\omega}(\omega)}=\left(\begin{array}{c}
U_{13}^{*} \\
U_{12}^{*}
\end{array}\right)\left(\rho_{\omega}(\lambda) I-\delta_{\omega}(\lambda) U_{11}\right)^{-*}\left(\rho_{\omega}(z) I-\delta_{\omega}(z) U_{11}\right)^{-1}\left(U_{13}, U_{12}\right)
\end{aligned}
$$

are immediate consequences of the unitarity of the operator $\mathbf{U}$ and imply in particular that the function $\Sigma$ is inner in $\mathbb{D}$. Applying the matrix identity

$$
\left(I+Y X^{-1} Z\right)^{-1}=I-Y(X+Z Y)^{-1} Z
$$

to the matrices

$$
X=\mathbf{Q}^{*} \Delta_{\omega}(\omega) \mathbf{Q}, \quad Y=\frac{\delta_{\omega}(z)}{\rho_{\omega}(z)} P^{\frac{1}{2}} H(\omega)^{*} \mathbf{Q}, \quad Z=\mathbf{Q}^{*} G(\omega)^{*} P^{\frac{1}{2}}
$$

and taking (9.8), (6.1), (6.19) and (6.21) into account, we get

$$
\begin{aligned}
\left(I-\frac{\delta_{\omega}(z)}{\rho_{\omega}(z)} U_{11}\right)^{-1}= & \left(I+\frac{\delta_{\omega}(z)}{\rho_{\omega}(z)} P^{\frac{1}{2}} H(\omega)^{*} \mathbf{Q}\left(\mathbf{Q}^{*} \Delta_{\omega}(\omega) \mathbf{Q}\right)^{-1} \mathbf{Q}^{*} G(\omega)^{*} P^{\frac{1}{2}}\right)^{-1} \\
= & I-\delta_{\omega}(z) P^{\frac{1}{2}} H(\omega)^{*} \mathbf{Q}\left\{\rho_{\omega}(z) \mathbf{Q}^{*} \Delta_{\omega}(\omega) \mathbf{Q}\right. \\
& \left.+\delta_{\omega}(z) \mathbf{Q}^{*} G(\omega)^{*} P H(\omega)^{*} \mathbf{Q}\right\}^{-1} \mathbf{Q}^{*} G(\omega)^{*} P^{\frac{1}{2}} \\
= & I-\delta_{\omega}(z) P^{\frac{1}{2}} H(\omega)^{*} \mathbf{Q}\left(\rho_{\omega}(\omega) \mathbf{Q}^{*} \Delta_{\omega}(z) \mathbf{Q}\right)^{-1} \mathbf{Q}^{*} G(\omega)^{*} P^{\frac{1}{2}} \\
= & I-\frac{\delta_{\omega}(z)}{\rho_{\omega}(\omega)} P^{\frac{1}{2}} H(\omega)^{*} \Delta_{\omega}(z)^{[-1]} G(\omega)^{*} P^{\frac{1}{2}}
\end{aligned}
$$

Using (6.20), (9.8) and (10.2) we obtain

$$
\begin{aligned}
U_{21}\left(I-\frac{\delta_{\omega}(z)}{\rho_{\omega}(z)} U_{11}\right)^{-1}= & \rho_{\omega}(\omega)^{\frac{1}{2}} C_{1} \Delta_{\omega}^{[-1]}(\omega) G(\omega)^{*} P^{\frac{1}{2}} \\
& \times\left(I-\frac{\delta_{\omega}(z)}{\rho_{\omega}(\omega)} P^{\frac{1}{2}} H(\omega)^{*} \Delta_{\omega}^{[-1]}(z) G(\omega)^{*} P^{\frac{1}{2}}\right) \\
= & \frac{\rho_{\omega}(z)}{\rho_{\omega}(\omega)^{\frac{1}{2}}} C_{1} \Delta_{\omega}^{[-1]}(z) G(\omega)^{*} P^{\frac{1}{2}}
\end{aligned}
$$

and

$$
\begin{aligned}
\left(I-\frac{\delta_{\omega}(z)}{\rho_{\omega}(z)} U_{11}\right)^{-1} U_{12}= & -\rho_{\omega}(\omega)^{\frac{1}{2}}\left(I-\frac{\delta_{\omega}(z)}{\rho_{\omega}(\omega)} P^{\frac{1}{2}} H(\omega)^{*} \Delta_{\omega}^{[-1]}(z) G(\omega)^{*} P^{\frac{1}{2}}\right) \\
& \times P^{\frac{1}{2}} H(\omega)^{*} \Delta_{\omega}^{[-1]}(\omega) C_{2}^{*} \\
= & -\frac{\rho_{\omega}(z)}{\rho_{\omega}(\omega)^{\frac{1}{2}}} P^{\frac{1}{2}} H(\omega)^{*} \Delta_{\omega}^{[-1]}(z) C_{2}^{*} .
\end{aligned}
$$


The direct substitution of (9.9) and the last three formulas into the entries of formula (10.1) leads to

$$
\begin{aligned}
\Sigma_{11}(z) & =U_{23}+\frac{\delta_{\omega}(z)}{\rho_{\omega}(z)} U_{21}\left(I-\frac{\delta_{\omega}(z)}{\rho_{\omega}(z)} U_{11}\right)^{-1} U_{13} \\
& =\left(\frac{\delta_{\omega}(z)}{\rho_{\omega}(z)} U_{21}\left(I-\frac{\delta_{\omega}(z)}{\rho_{\omega}(z)} U_{11}\right)^{-1}, I_{p}\right)\left(\begin{array}{c}
U_{13} \\
U_{23}
\end{array}\right) \\
& =\left(\frac{\delta_{\omega}(z)}{\rho_{\omega}(\omega)^{\frac{1}{2}}} C_{1} \Delta_{\omega}^{[-1]}(z) G(\omega)^{*} P^{\frac{1}{2}}, I_{p}\right) W_{2}^{\perp} \\
\Sigma_{12}(z) & =U_{22}+\frac{\delta_{\omega}(z)}{\rho_{\omega}(z)} U_{21}\left(I-\frac{\delta_{\omega}(z)}{\rho_{\omega}(z)} U_{11}\right)^{-1} U_{12} \\
& =\rho_{\omega}(\omega) C_{1} \Delta_{\omega}^{[-1]}(\omega) C_{2}^{*}-\delta_{\omega}(z) C_{1} \Delta_{\omega}^{[-1]}(z) G(\omega)^{*} P H(\omega)^{*} \Delta_{\omega}^{[-1]}(\omega) C_{2}^{*} \\
& =\rho_{\omega}(z) C_{1} \Delta_{\omega}^{[-1]}(z) C_{2}^{*} \\
\Sigma_{21}(z) & =\frac{\delta_{\omega}(z)}{\rho_{\omega}(z)} U_{31}\left(I-\frac{\delta_{\omega}(z)}{\rho_{\omega}(z)} U_{11}\right)^{-1} U_{13} \\
& =\frac{\delta_{\omega}(z)}{\rho_{\omega}(z)}\left(W_{1}^{\perp}\right)^{*}\left(\begin{array}{c}
I_{n} \\
0
\end{array}\right)\left(I-\frac{\delta_{\omega}(z)}{\rho_{\omega}(\omega)} P^{\frac{1}{2}} H(\omega)^{*} \Delta_{\omega}^{[-1]}(z) G(\omega)^{*} P^{\frac{1}{2}}\right)\left(I_{n}, 0\right) W_{2}^{\perp}
\end{aligned}
$$

and

$$
\begin{aligned}
& \Sigma_{22}(z)=U_{32}+\frac{\delta_{\omega}(z)}{\rho_{\omega}(z)} U_{31}\left(I-\frac{\delta_{\omega}(z)}{\rho_{\omega}(z)} U_{11}\right)^{-1} U_{12} \\
& =\left(\begin{array}{ll}
U_{31}, & U_{32}
\end{array}\right)\left(\begin{array}{c}
\frac{\delta_{\omega}(z)}{\rho_{\omega}(z)}\left(I-\frac{\delta_{\omega}(z)}{\rho_{\omega}(z)} U_{11}\right)^{-1} U_{12} \\
I_{q}
\end{array}\right) \\
& =\left(W_{1}^{\perp}\right)^{*}\left(\begin{array}{c}
-\frac{\delta_{\omega}(z)}{\rho_{\omega}(\omega)^{\frac{1}{2}}} P^{\frac{1}{2}} H(\omega)^{*} \Delta_{\omega}^{[-1]}(z) C_{2}^{*} \\
I_{q}
\end{array}\right) .
\end{aligned}
$$

Since $\operatorname{rank} P=r$, it follows from (9.1) that the isometric matrices $W_{1}^{\perp}$ and $W_{2}^{\perp}$ can be chosen in the form

$$
W_{1}^{\perp}=\left(\begin{array}{cc}
X & Y_{1} \\
0 & Z_{1}
\end{array}\right) \quad \text { and } \quad W_{2}^{\perp}=\left(\begin{array}{cc}
X & Y_{2} \\
0 & Z_{2}
\end{array}\right)
$$

where $X \in \mathbb{C}^{n \times(n-r)}$ is an isometric matrix whose columns are an orthonormal basis for Ker P. Substituting (10.7) into (10.3), (10.5) and (10.6) we get

$$
\Sigma_{11}(z)=\left(0, \Psi_{11}(z)\right), \quad \Sigma_{21}(z)=\left(\begin{array}{cc}
\frac{\delta_{\omega}(z)}{\rho_{\omega}(z)} I_{n-r} & 0 \\
0 & \Psi_{21}(z)
\end{array}\right), \quad \Sigma_{22}(z)=\left(\begin{array}{c}
0 \\
\Psi_{22}(z)
\end{array}\right),
$$

where

$$
\begin{aligned}
\Psi_{11}(z) & =Z_{2}+\rho_{\omega}(\omega)^{-\frac{1}{2}} \delta_{\omega}(z) C_{1} \Delta_{\omega}^{[-1]}(z) G(\omega)^{*} P^{\frac{1}{2}} Y_{2} \\
\Psi_{21}(z) & =\frac{\delta_{\omega}(z)}{\rho_{\omega}(z)} Y_{1}^{*}\left(I-\frac{\delta_{\omega}(z)}{\rho_{\omega}(\omega)} P^{\frac{1}{2}} H(\omega)^{*} \Delta_{\omega}^{[-1]}(z) G(\omega)^{*} P^{\frac{1}{2}}\right) Y_{2} \\
\Psi_{22}(z) & =Z_{1}^{*}-\rho_{\omega}(\omega)^{-\frac{1}{2}} \delta_{\omega}(z) Y_{1}^{*} P^{\frac{1}{2}} H(\omega)^{*} \Delta_{\omega}^{[-1]}(z) C_{2}^{*} .
\end{aligned}
$$


Upon writing the parameter $\mathcal{E}(z) \in \mathcal{S}^{p^{\prime} \times q^{\prime}}$ in $(9.17)$ in the block form

$$
\mathcal{E}(z)=\left(\begin{array}{ll}
\mathcal{E}_{11}(z) & \mathcal{E}_{12}(z) \\
\mathcal{E}_{21}(z) & \mathcal{E}_{22}(z)
\end{array}\right): \quad\left(\begin{array}{l}
\mathbb{C}^{n-r} \\
\mathbb{C}^{q-\nu}
\end{array}\right) \rightarrow\left(\begin{array}{l}
\mathbb{C}^{n-r} \\
\mathbb{C}^{p-\nu}
\end{array}\right)
$$

and using the block decompositions (10.8) of $\Sigma_{j k}$ and an inversion formula based on Schur complements, one can check that

$$
\Sigma_{11}(z) \mathcal{E}(z)\left(I_{q}-\Sigma_{21}(z) \mathcal{E}(z)\right)^{-1} \Sigma_{22}(z)=\Psi_{11}(z) \widehat{\mathcal{E}}(z)\left(I_{q}-\Psi_{21}(z) \widehat{\mathcal{E}}(z)\right)^{-1} \Psi_{22}(z),
$$

where

$$
\widehat{\mathcal{E}}(z)=\mathcal{E}_{22}(z)+\frac{\delta_{\omega}(z)}{\rho_{\omega}(z)} \mathcal{E}_{21}(z)\left(I-\frac{\delta_{\omega}(z)}{\rho_{\omega}(z)} \mathcal{E}_{11}(z)\right)^{-1} \mathcal{E}_{12}(z)
$$

The function $\widehat{\mathcal{E}}$ is the Redheffer transform based the transfer function $\left(\begin{array}{cc}\mathcal{E}_{21}(z) & \mathcal{E}_{22}(z) \\ \mathcal{E}_{11}(z) & \mathcal{E}_{12}(z)\end{array}\right) \in$ $\mathcal{S}^{q^{\prime} \times p^{\prime}}$ of the parameter $\frac{\delta_{\omega}(z)}{\rho_{\omega}(z)} I_{n-r} \in \mathcal{S}^{(n-r) \times(n-r)}$. Therefore, $\widehat{\mathcal{E}} \in \mathcal{S}^{(p-\nu) \times(q-\nu)}$. On the other hand, the set of all functions $\widehat{\mathcal{E}}$ of the form (10.14) (where $\mathcal{E}$ partitioned as in (10.12) varies over $\left.\mathcal{S}^{q^{\prime} \times p^{\prime}}\right)$ covers all $\widehat{\mathcal{E}} \in \mathcal{S}^{(p-\nu) \times(q-\nu)}$. Therefore, upon setting

$$
\Psi_{12}(z):=\Sigma_{12}(z)=\rho_{\omega}(z) C_{1} \Delta_{\omega}^{[-1]}(z) C_{2}^{*},
$$

and substituting (10.13) into (9.17) we obtain

Theorem 10.1 All the solutions $S$ of the $\mathbf{~ B B I P}$ are parametrized by the Redheffer transformation

$$
S(z)=\Psi_{12}(z)+\Psi_{11}(z) \widehat{\mathcal{E}}(z)\left(I_{q}-\Psi_{21}(z) \widehat{\mathcal{E}}(z)\right)^{-1} \Psi_{22}(z),
$$

where $\Psi=\left(\begin{array}{ll}\Psi_{11} & \Psi_{12} \\ \Psi_{21} & \Psi_{22}\end{array}\right)$ is the inner function defined by (10.9)-(10.11) and (10.15) and $\widehat{\mathcal{E}}$ is a free independent parameter varying over $\mathcal{S}^{(p-\nu) \times(q-\nu)}$.

To show that the function $\Psi$ is inner it suffices to note that in view of (10.8),

$$
\Sigma(z)=\left(\begin{array}{cc}
\Sigma_{11}(z) & \Sigma_{12}(z) \\
\Sigma_{21}(z) & \Sigma_{22}(z)
\end{array}\right)=\left(\begin{array}{ccc}
0 & \Psi_{11}(z) & \Psi_{12}(z) \\
\frac{\delta_{\omega}(z)}{\rho_{\omega}(z)} I_{n-r} & 0 & 0 \\
0 & \Psi_{21}(z) & \Psi_{22}(z)
\end{array}\right)
$$

and to use the fact that $\Sigma(z)$ is inner.

It turns out that the function $\Psi_{12}$ is a very special solution of the aBIP with important extremal properties. The next lemma establishes some relations which will be used in Section 11.

Lemma 10.2 Let $\omega \in \mathbb{D}$ and let $\Psi_{12}$ be the function given by (10.4). Then

$$
\begin{aligned}
\left(I_{p},-\Psi_{12}(z)\right) C G^{-1}(z) & =C_{1} \Delta_{\omega}^{[-1]}(z) G(\omega)^{*} P \\
\left(-\Psi_{12}(z)^{*}, I_{q}\right) C H(z)^{-*} & =C_{2} \Delta_{\omega}^{[-1]}(z)^{*} H(\omega) P
\end{aligned}
$$


and for every choice of $\zeta \in \mathbb{T}$,

$$
\begin{aligned}
& G(\zeta)^{-*} C^{*}\left(\begin{array}{cc}
I_{p} & -\Psi_{12}(\zeta) \\
-\Psi_{12}(\zeta)^{*} & I_{q}
\end{array}\right) C G^{-1}(\zeta) \\
& =\frac{\rho_{\omega}(\omega)}{\left|\rho_{\omega}(\zeta)\right|^{2}} P-\frac{\bar{\zeta}}{\rho_{\omega}(\zeta)^{*}} P G(\omega) \Delta_{\omega}^{[-1]}(\zeta)^{*} H(\omega) P-\frac{1}{\delta_{\omega}(\zeta)^{*}} P H(\omega)^{*} \Delta_{\omega}^{[-1]}(\zeta) G(\omega)^{*} P .
\end{aligned}
$$

Proof: First we note that in view of (9.2),

$$
P G(\omega) \mathbf{P}_{\mathcal{K}}=P H(\omega)^{*} \mathbf{P}_{\mathcal{K}}=0, \quad C_{1} \mathbf{P}_{\mathcal{K}}=0 \quad \text { and } \quad C_{2} \mathbf{P}_{\mathcal{K}}=0
$$

where $\mathbf{P}_{\mathcal{K}}$ denotes the orthogonal projection of $\mathbb{C}^{n}$ onto $\mathcal{K}=\operatorname{Ker} \Delta_{\omega}(\omega)$. Using (6.1) and (6.24) we get

$$
\rho_{\omega}(z) \Delta_{\omega}^{[-1]}(z) C_{2}^{*} C_{2}=I_{n}-\mathbf{P}_{\mathcal{K}}-\Delta_{\omega}^{[-1]}(z) G(\omega)^{*} P G(z) .
$$

Multiplying both sides of the latter equality by $C_{1}$ from the left and taking (10.15) into account, we obtain

$$
\Psi_{12}(z) C_{2}=C_{1}-C_{1} \Delta_{\omega}^{[-1]}(z) G(\omega)^{*} P G(z),
$$

which is equivalent to (10.17). The equality (10.18) is proved in much the same way with the help of the relation

$$
\rho_{\omega}(z) C_{1}^{*} C_{1} \Delta_{\omega}^{[-1]}(z)=I_{n}-\mathbf{P}_{\mathcal{K}}-H(z) P H(\omega)^{*} \Delta_{\omega}^{[-1]}(z)
$$

Next, by (10.17), (10.18) and (3.15),

$$
\begin{aligned}
& G(\zeta)^{-*} C^{*}\left(\begin{array}{cc}
I_{p} & -\Psi_{12}(\zeta) \\
-\Psi_{12}(\zeta)^{*} & I_{q}
\end{array}\right) C G^{-1}(\zeta) \\
& =G(\zeta)^{-*} C_{1}^{*} C_{1} \Delta_{\omega}^{[-1]}(\zeta) G(\omega)^{*} P+\bar{\zeta} G(\zeta)^{-*} C_{2}^{*} C_{2} \Delta_{\omega}^{[-1]}(\zeta)^{*} H(\omega) P .
\end{aligned}
$$

In view of (10.20) and (10.21),

$$
\bar{\zeta} G(\zeta)^{-*} C_{2}^{*} C_{2} \Delta_{\omega}^{[-1]}(\zeta)^{*} H(\omega) P=\frac{\bar{\zeta}}{\rho_{\omega}(\zeta)^{*}}\left\{G(\zeta)^{-*}-P G(\omega) \Delta_{\omega}^{[-1]}(\zeta)^{*}\right\} H(\omega) P,
$$

whereas (10.22) implies that

$$
G(\zeta)^{-*} C_{1}^{*} C_{1} \Delta_{\omega}^{[-1]}(\zeta) G(\omega)^{*} P=\frac{1}{\rho_{\omega}(\zeta)}\left\{G(\zeta)^{-*}-\zeta P H(\omega)^{*} \Delta_{\omega}^{[-1]}(\zeta)\right\} G(\omega)^{*} P .
$$

Finally, we get (10.19) by substituting (10.24) and (10.25) into the right hand side of (10.23) and making use of the identity

$$
\frac{1}{\rho_{\omega}(\zeta)} G(\omega)^{*}+\frac{\bar{\zeta}}{\rho_{\omega}(\zeta)^{*}} H(\omega)=\frac{\rho_{\omega}(\omega)}{\left|\rho_{\omega}(\zeta)\right|^{2}} G(\zeta)^{*}
$$


Lemma 10.3 Let $\Psi_{11}$ and $\Psi_{22}$ be the functions defined by (10.9) and (10.11), respectively. Then

$$
C_{1}^{*} \Psi_{11}(z)=H(z) P^{\frac{1}{2}} \Upsilon_{\omega}(z) Y_{2} \quad \text { and } \quad \Psi_{22}(z) C_{2}=-Y_{1}^{*} \Upsilon_{\omega}(z) P^{\frac{1}{2}} G(z)
$$

where

$$
\Upsilon_{\omega}(z)=\frac{\rho_{\omega}(\omega)^{\frac{1}{2}}}{\rho_{\omega}(z)}\left\{I-\frac{\delta_{\omega}(z)}{\rho_{\omega}(\omega)} P^{\frac{1}{2}} H(\omega)^{*} \Delta_{\omega}^{[-1]}(z) G(\omega)^{*} P^{\frac{1}{2}}\right\}
$$

Proof: Substituting the block decompositions (9.1) and (10.7) of $W_{j}$ and $W_{j}^{\perp}(j=1,2)$ into the two last relations in (9.7), we get in particular,

$$
\rho_{\omega}(\omega)^{\frac{1}{2}} C_{1}^{*} Z_{2}-H(\omega) P^{\frac{1}{2}} Y_{2}=0 \quad \text { and } \quad \rho_{\omega}(\omega)^{\frac{1}{2}} Z_{1}^{*} C_{2}+Y_{1}^{*} P^{\frac{1}{2}} G(\omega)=0 .
$$

Using (10.9) and the first relation in (10.28) we obtain

$$
C_{1}^{*} \Psi_{11}(z)=\rho_{\omega}(\omega)^{-\frac{1}{2}}\left\{H(\omega)+\delta_{\omega}(z) C_{1}^{*} C_{1} \Delta_{\omega}^{[-1]}(z) G(\omega)^{*}\right\} P^{\frac{1}{2}} Y_{2}
$$

However, by (10.22) and (10.20),

$$
C_{1}^{*} C_{1} \Delta_{\omega}^{[-1]}(z) G(\omega)^{*} P^{\frac{1}{2}}=\frac{1}{\rho_{\omega}(z)}\left\{G(\omega)^{*}-H(z) P H(\omega)^{*} \Delta_{\omega}^{[-1]}(z) G(\omega)^{*}\right\} P^{\frac{1}{2}},
$$

whereas

$$
\frac{\delta_{\omega}(z)}{\rho_{\omega}(z)} G(\omega)^{*}+H(\omega)=\frac{\rho_{\omega}(\omega)}{\rho_{\omega}(z)} H(z) .
$$

Finally, upon substituting (10.30) into (10.29) and taking advantage of (10.31), we get the first relation in (10.26) with $\Upsilon_{\omega}$ defined by (10.27). The second relation in (10.26) is proved quite similarly.

\section{Maximum entropy}

It is well known that for a large class of problems whose solutions can be expressed as a linear fractional transformation of the Schur class $\mathcal{S}^{p \times q}$ based on a $J$-inner function there is exactly one solution which maximizes the $\omega$-entropy integral

$$
\mathbf{E}_{\omega}(S)=\int_{0}^{2 \pi} \ln \operatorname{det}\left(I_{p}-S\left(e^{i t}\right) S\left(e^{i t}\right)^{*}\right) d \sigma_{\omega}(t)
$$

where

$$
\omega \in \mathbb{D} \quad \text { and } \quad d \sigma_{\omega}(t)=\frac{1}{2 \pi} \frac{1-|\omega|^{2}}{\left|e^{i t}-\omega\right|^{2}} d t .
$$

For additional information see e.g., [13, Section 11] and the references cited therein (especially [7]). In particular, if the Pick matrix $P$ of the $\mathbf{a B I P}$ is strictly positive, then the linear fractional representation (7.13) of the set of all solutions of the aBIP is based on the $J_{-}$ inner function $\Theta$ given in (7.6) and the parameter $\mathcal{E}$ is an arbitrary element in the Schur class $\mathcal{S}^{p \times q}$ (i.e., $\nu=0$ in (7.14)). In this setting we have the following conclusion: 
Theorem 11.1 Let (1.5) be in force, let $P>0$ and let $S$ be of the form (7.13) for some $\mathcal{E} \in \mathcal{S}^{p \times q}$, where the mvf $\Theta$ is given by (7.6). Then $\theta_{21}$ is analytic at each point $\omega \in \mathbb{D}$ in the domain of analyticity of $\theta_{22}$ and

$$
\mathbf{E}_{\omega}(S) \leq \ln \operatorname{det}\left(\theta_{22}(\omega) \theta_{22}(\omega)^{*}-\theta_{21}(\omega) \theta_{21}(\omega)^{*}\right)^{-1}
$$

with equality if and only if ${ }^{1}$

$$
S(z)=S_{\max }(z)=\left(\theta_{11}(z) \theta_{21}(\omega)^{*}-\theta_{12}(z) \theta_{22}(\omega)^{*}\right)\left(\theta_{21}(z) \theta_{21}(\omega)^{*}-\theta_{22}(z) \theta_{22}(\omega)^{*}\right)^{-1},
$$

i.e., the inequality (11.2) holds for every solution $S$ of the $\mathrm{aBIP}$ with equality if and only if $S$ is the function corresponding via (7.13) to the parameter

$$
\mathcal{E}_{\max }(z) \equiv-\theta_{21}(\omega)^{*} \theta_{22}(\omega)^{-*} .
$$

Theorem 11.1 is not directly applicable if $P$ is singular. One reason is that the expression on the right hand side of (11.3) is a strictly contractive matrix (this follows from (7.12)) and therefore cannot be used as a parameter in the transformation (7.13) if $r<n$. Another reason is that

$$
\operatorname{det}\left(I_{p}-S(z) S(z)^{*}\right) \equiv 0
$$

for each solution $S$ of the degenerate problem and therefore the entropy integral (11.1) does not exist. To overcome these difficulties we shall use a generalized definition of entropy which was introduced by Y. Inouye in [19] to study regular random processes with degenerate rank. The idea is to use a sum of principal minors of appropriate order instead of the determinant in (11.1). To clarify this, let $\mathbf{D}_{r}[T]$ denote the sum of principal minors of a matrix $T \in \mathbb{C}^{m \times m}$ for $r \leq m$. The following result is an easy consequence of the Binet-Cauchy formula [18].

Lemma 11.2 If $F \in \mathbb{C}^{m \times n}, G \in \mathbb{C}^{n \times m}$ and $r \leq \min (m, n)$, then

$$
\mathbf{D}_{r}[F G]=\mathbf{D}_{r}[G F] .
$$

The number $\mathbf{D}_{r}[T]$ can be expressed more explicitly for a Hermitian matrix $T$ of rank $r$.

Lemma 11.3 Let $T \in \mathbb{C}^{m \times m}$ be a Hermitian matrix with $\operatorname{rank} T=r \leq m$. Then $\mathbf{D}_{r}[T]$ is equal to the product of all the nonzero eigenvalues of $T$ (counting multiplicities).

Proof: Let $\lambda_{1}(T), \ldots, \lambda_{r}(T)$ be the nonzero eigenvalues of $T$ and let $U \in \mathbb{C}^{m \times m}$ be a unitary matrix such that

$$
U T U^{*}=\left(\begin{array}{cc}
\operatorname{diag}\left(\lambda_{j}(T)\right)_{j=1}^{r} & 0 \\
0 & 0
\end{array}\right) .
$$

\footnotetext{
${ }^{1}$ The inequality (11.2) corrects the misprinted stars in inequality (11.2) of [13].
} 
Then, by (11.4),

$$
\mathbf{D}_{r}[T]=\mathbf{D}_{r}\left[T U^{*} U\right]=\mathbf{D}_{r}\left[U T U^{*}\right]=\operatorname{det} \operatorname{diag}\left(\lambda_{j}(T)\right)_{j=1}^{r}=\prod_{j=1}^{n} \lambda_{j}(T) .
$$

Following [19], we shall consider entropy integrals of the form

$$
\mathbf{E}_{\omega}^{(j)}(S)=\int_{0}^{2 \pi} \ln \mathbf{D}_{p-j}\left[I_{p}-S\left(e^{i t}\right) S\left(e^{i t}\right)^{*}\right] d \sigma_{\omega}(t), \quad d \sigma_{\omega}(t)=\frac{1}{2 \pi} \frac{1-|\omega|^{2}}{\left|e^{i t}-\omega\right|^{2}} d t,
$$

where $\omega \in \mathbb{D}$ and $j$ is an integer between 0 and $p-1$. If $j=0$, then formula (11.5) coincides with (11.1). If $P$ is singular and $\nu$ is given by (1.11), then the integral (11.5) diverges to minus infinity for $j \leq \nu-1$ for every solution $S$ of the aBIP. However, if $j=\nu$, then there exists solutions for which this integral converges and the problem of finding its maximum value makes sense. In this section we obtain an analogue of Theorem 11.1 for $j=\nu$ and with the integral (11.5) in place of (11.1).

In order to obtain this analogue, it is convenient to express the solutions $S$ of the aBIP in terms of a Redheffer transform of the form (10.16). We begin with a formulation for a general inner mvf $\Psi$ and subsequently, in the next theorem, observe that if $\Psi$ is normalized as in (10.9)-(10.11) and (10.15) at the same point $\omega$ as in (11.5), then the main conclusions simplify.

Theorem 11.4 Let $\Psi=\left(\begin{array}{ll}\Psi_{11} & \Psi_{12} \\ \Psi_{21} & \Psi_{22}\end{array}\right):\left(\begin{array}{l}\mathbb{C}^{p-\nu} \\ \mathbb{C}^{q}\end{array}\right) \rightarrow\left(\begin{array}{l}\mathbb{C}^{p} \\ \mathbb{C}^{q-\nu}\end{array}\right)$ be an inner mvf in which the block $\Psi_{21}$ is strictly contractive in $\mathbb{D}$ and let $S$ be of the form (10.16) for some $\widehat{\mathcal{E}} \in \mathcal{S}^{(p-\nu) \times(q-\nu)}$. Then for each $\omega \in \mathbb{D}$,

$$
\mathbf{E}_{\omega}^{(\nu)}(S) \leq \int_{0}^{2 \pi} \ln \operatorname{det}\left[I_{p-\nu}-\Psi_{21}\left(e^{i t}\right)^{*} \Psi_{21}\left(e^{i t}\right)\right] d \sigma_{\omega}(t)+\ln \operatorname{det}\left[I_{p-\nu}-\Psi_{21}(\omega)^{*} \Psi_{21}(\omega)\right]^{-1}
$$

with equality if and only if

$$
S(z)=S_{\max }(z)=\Psi_{12}(z)+\Psi_{11}(z) \Psi_{21}(\omega)^{*}\left(I_{q}-\Psi_{21}(z) \Psi_{21}(\omega)^{*}\right)^{-1} \Psi_{22}(z)
$$

or, equivalently, if and only if $S$ is the function corresponding via (10.16) to the parameter

$$
\widehat{\mathcal{E}}_{\max }(z) \equiv \Psi_{21}(\omega)^{*} .
$$

Proof: Let $S$ be of the form (10.16) for some $\widehat{\mathcal{E}} \in \mathcal{S}^{(p-\nu) \times(q-\nu)}$. By Theorem 8.5, the transfer function $\Psi$ is inner in $\mathbb{D}$ and so, in particular,

$$
\Psi_{11}\left(e^{i t}\right)^{*} \Psi_{11}\left(e^{i t}\right)=I_{p-\nu}-\Psi_{21}\left(e^{i t}\right)^{*} \Psi_{21}\left(e^{i t}\right) .
$$

On the other hand, by Lemma 8.3,

$$
I_{p}-S(\zeta) S(\zeta)^{*}=\Psi_{11}(\zeta) K(\zeta) \Psi_{11}(\zeta)^{*}
$$


at almost every point of the unit circle $\mathbb{T}$, where

$$
K:=\left(I-\widehat{\mathcal{E}} \Psi_{21}\right)^{-1}\left(I-\widehat{\mathcal{E}} \widehat{\mathcal{E}}^{*}\right)\left(I-\widehat{\mathcal{E}} \Psi_{21}\right)^{-*}
$$

for short. Therefore,

$$
\begin{aligned}
\mathbf{D}_{p-\nu}\left[I_{p}-S\left(e^{i t}\right) S\left(e^{i t}\right)^{*}\right] & =\mathbf{D}_{p-\nu}\left[\Psi_{11}\left(e^{i t}\right) K\left(e^{i t}\right) \Psi_{11}\left(e^{i t}\right)^{*}\right] \\
& =\mathbf{D}_{p-\nu}\left[K\left(e^{i t}\right) \Psi_{11}\left(e^{i t}\right)^{*} \Psi_{11}\left(e^{i t}\right)\right] \\
& =\mathbf{D}_{p-\nu}\left[K\left(e^{i t}\right)\left(I_{p-\nu}-\Psi_{21}\left(e^{i t}\right)^{*} \Psi_{21}\left(e^{i t}\right)\right)\right] \\
& =\operatorname{det} K\left(e^{i t}\right) \times \operatorname{det}\left(I_{p-\nu}-\Psi_{21}\left(e^{i t}\right)^{*} \Psi_{21}\left(e^{i t}\right)\right) .
\end{aligned}
$$

The rest of the proof amounts to estimating $\int \ln \operatorname{det} K\left(e^{i t}\right) d \sigma_{\omega}(t)$. The argument is adapted from [13, Section 11], which in turn is adapted from [7]. To begin with, since $\widehat{\mathcal{E}} \Psi_{21}$ is a Schur function which is strictly contractive in $\mathbb{D}$, the function $\left(I_{p-\nu}-\widehat{\mathcal{E}} \Psi_{21}\right)$ is outer and

$$
\begin{aligned}
\int_{0}^{2 \pi} \ln \operatorname{det}\left(I_{p-\nu}-\widehat{\mathcal{E}}\left(e^{i t}\right) \Psi_{21}\left(e^{i t}\right)\right) d \sigma_{\omega}(t) & =\ln \operatorname{det}\left(I_{p-\nu}-\widehat{\mathcal{E}}(\omega) \Psi_{21}(\omega)\right) \\
& =\int_{0}^{2 \pi} \ln \operatorname{det}\left(I_{p-\nu}-\widehat{\mathcal{E}}\left(e^{i t}\right) \Psi_{21}(\omega)\right) d \sigma_{\omega}(t) .
\end{aligned}
$$

Thus,

$$
\begin{aligned}
& \int_{0}^{2 \pi} \ln \operatorname{det} K\left(e^{i t}\right) d \sigma_{\omega}(t) \\
& =\int_{0}^{2 \pi} \ln \operatorname{det}\left(I_{p-\nu}-\widehat{\mathcal{E}}\left(e^{i t}\right) \Psi_{21}(\omega)\right)^{-1}\left(I-\widehat{\mathcal{E}}\left(e^{i t}\right) \widehat{\mathcal{E}}\left(e^{i t}\right)^{*}\right)\left(I_{p-\nu}-\widehat{\mathcal{E}}\left(e^{i t}\right) \Psi_{21}(\omega)\right)^{-*} d \sigma_{\omega}(t) .
\end{aligned}
$$

The following matrix identity

$$
\left(I_{k}-F G\right)\left(I_{k}-G^{*} G\right)^{-1}\left(I_{k}-G^{*} F^{*}\right)=I_{k}-F F^{*}+\left(F-G^{*}\right)\left(I_{\ell}-G G^{*}\right)^{-1}\left(F^{*}-G\right)
$$

holds for any contractive $F \in \mathbb{C}^{k \times \ell}$ and strictly contractive $G \in \mathbb{C}^{\ell \times k}$; it is easily verified by straightforward calculation. As a consequence we get the inequality

$$
\left(I_{k}-F G\right)^{-1}\left(I_{k}-F F^{*}\right)\left(I_{k}-G^{*} F^{*}\right)^{-1} \leq\left(I_{k}-G^{*} G\right)^{-1}
$$

with equality if and only if $F=G^{*}$. Applying (11.10) to $F=\widehat{\mathcal{E}}\left(e^{i t}\right)$ and $G=\Psi_{21}(\omega)$ we conclude that

$$
\int_{0}^{2 \pi} \ln \operatorname{det} K\left(e^{i t}\right) d \sigma_{\omega}(t) \leq \ln \operatorname{det}\left(I_{p}-\Psi_{21}(\omega)^{*} \Psi_{21}(\omega)\right)^{-1}
$$

with equality if and only if (11.7) holds. The assertion of the theorem follows immediately from (11.9) and (11.11).

As a corollary we obtain the following generalization of Theorem 11.1. 
Theorem 11.5 Let (1.5) be in effect, let $P$ be a nonnegative solution of the Lyapunov-Stein equation (1.8) and let $\nu$ and $\Psi_{21}$ be defined by (1.11) and (10.10), respectively. Then for each solution $S$ of the $\operatorname{aBIP}(M, N, P, C)$,

$$
\mathbf{E}_{\omega}^{(\nu)}(S) \leq \int_{0}^{2 \pi} \ln \operatorname{det}\left[I_{p-\nu}-\Psi_{21}\left(e^{i t}\right)^{*} \Psi_{21}\left(e^{i t}\right)\right] d \sigma_{\omega}(t)
$$

with equality if and only if $S$ is taken equal to

$$
S_{\max }(z)=\rho_{\omega}(z) C_{1} \Delta_{\omega}^{[-1]}(z) C_{2}^{*}
$$

For the proof it is enough to note that if $\Psi$ is normalized as in (10.9)-(10.11) and (10.15), then in particular, $\Psi_{21}(\omega)=0$. Note also that the extremal function $S_{\max }$ is unique and therefore, the formula (11.12) can be obtained from (11.6) if $\Psi$ is normalized at a point $\widetilde{\omega}$ which is different from $\omega$. The direct calculation, however, is rather complicated.

\section{On maximum determinant extension problems}

In this section we shall study the problem of completing a partially specified structured matrix in such a way as to maximize its determinant over the set of all positive semidefinite completions. The structure of the unknown matrix will be defined by a Lyapunov-Stein equation and thus the problem can be formulated in terms of finding the solution of a partially specified Lyapunov-Stein equation with maximum determinant. Extremal problems of this sort with strictly positive data were considered in [17] for the classical Nevanlinna-Pick and Carathéodory-Fejér interpolation problems and in [15] for the general aBIP.

It can happen that the given entries of the partially specified matrix are such that every positive semidefinite completion will be degenerate (i.e., will have zero determinant). To make this extension problem meaningful for such cases also we shall maximize a sum of principal minors of appropriate size (much as in the previous section) instead of the determinant.

Lemma 12.1 Let $P \in \mathbb{C}^{n \times n}$ be a nonnegative matrix with rank $P=r \leq n$ and let

$$
\widehat{P}=\left(\begin{array}{ll}
P & q \\
q^{*} & d
\end{array}\right) \geq 0 \quad\left(d \in \mathbb{C}^{\kappa \times \kappa}, q \in \mathbb{C}^{n \times \kappa}\right)
$$

be its extension. Then

$$
\mathbf{D}_{r+\kappa}[\widehat{P}]=\mathbf{D}_{r}[P] \times \operatorname{det}\left(d-q^{*} P^{[-1]} q\right)
$$

where $P^{[-1]}$ is the Moore-Penrose pseudoinverse of $P$.

Proof: Let $U \in \mathbb{C}^{n \times n}$ be a unitary matrix such that

$$
U^{*} P U=\left(\begin{array}{cc}
0 & 0 \\
0 & P_{r}
\end{array}\right), \quad P_{r}>0
$$


By definition, the Moore-Penrose pseudoinverse of $P$ is given by

$$
P^{[-1]}=U\left(\begin{array}{cc}
0 & 0 \\
0 & P_{r}^{-1}
\end{array}\right) U^{*} .
$$

Multiplying (12.1) by the matrix $\mathbf{U}=\left(\begin{array}{cc}U & 0 \\ 0 & I_{\kappa}\end{array}\right)$ from the right, by its adjoint from the left and using (12.3) we get

$$
\mathbf{U}^{*} \widehat{P} \mathbf{U}=\left(\begin{array}{ccc}
0 & 0 & U^{*} q \\
0 & P_{r} & \\
q^{*} U & d
\end{array}\right)
$$

Therefore,

$$
U^{*} q=\left(\begin{array}{c}
0 \\
q_{r}
\end{array}\right) \quad \text { for some } \quad q_{r} \in \mathbb{C}^{r \times \kappa}
$$

and

$$
\begin{aligned}
\mathbf{D}_{r+\kappa}[\widehat{P}] & =\mathbf{D}_{r+\kappa}\left[\mathbf{U}^{*} P \mathbf{U}\right] \\
& =\operatorname{det}\left(\begin{array}{cc}
P_{r} & q_{r} \\
q_{r}^{*} & d
\end{array}\right)=\operatorname{det} P_{r} \times \operatorname{det}\left(d-q_{r}^{*} P_{r}^{-1} q_{r}\right) .
\end{aligned}
$$

But this is clearly the same as (12.2), since

$$
q_{r}^{*} P_{r}^{-1} q_{r}=q^{*} P^{[-1]} q .
$$

Let $M, N \in \mathbb{C}^{n \times n}$ satisfy the condition (1.5), let $P \in \mathbb{C}^{n \times n}$ be a positive semidefinite solution of the Lyapunov-Stein equation (1.10) with $\operatorname{rank} P=r \leq n$ and let

$$
\begin{array}{rlrl}
\widehat{M} & =\left(\begin{array}{cc}
M & u \\
0 & I_{\kappa}
\end{array}\right), & \widehat{N} & =\left(\begin{array}{cc}
N & v \\
0 & \bar{\omega} I_{\kappa}
\end{array}\right) \quad(|\omega|<1), \\
\widehat{C}_{1}=\left(C_{1}, c_{1}\right), & \widehat{C}_{2}=\left(C_{2}, x\right) & \left(c_{1} \in \mathbb{C}^{p \times \kappa}, x \in \mathbb{C}^{q \times \kappa}\right) .
\end{array}
$$

Problem 12.2 Find those positive semidefinite solutions $\widehat{P}=\widehat{P}(x)$ of the Lyapunov-Stein equation

$$
\widehat{M}^{*} \widehat{P} \widehat{M}-\widehat{N}^{*} \widehat{P} \widehat{N}=\widehat{C}_{1}^{*} \widehat{C}_{1}-\widehat{C}_{2}^{*} \widehat{C}_{2}
$$

which attain the maximum value of $\mathbf{D}_{r+\kappa}(\widehat{P})$.

By Lemma 12.1, it suffices to maximize the value of $\operatorname{det}\left(d-q^{*} P^{[-1]} q\right)$. Substituting the decompositions (12.1) and (12.4) into (12.5) we get

$$
d=\frac{1}{\rho_{\omega}(\omega)}\left\{v^{*} P v-u^{*} P u-q^{*}(u-\omega v)-\left(u^{*}-\bar{\omega} v^{*}\right) q+c_{1}^{*} c_{1}-x^{*} x\right\}
$$

and

$$
G(\omega)^{*} q=C_{1}^{*} c_{1}-C_{2}^{*} x+N^{*} P v-M^{*} P u .
$$

Thus, it is easily seen that if $G(\omega)$ is invertible, then the entries $q$ and $d$ of the extended matrix $\widehat{P}$ are uniquely (and explicitly) defined by $\widehat{M}, \widehat{N}$ and $\widehat{C}$. In this case, $\widehat{P}(x)$ is the mvf 
of the matrix argument $x$ and Problem 12.2 can be successfully solved using purely algebraic methods, as was done in [15] and [17]. Here we do not require the invertibility of $G(\omega)$; an explicit expression for $q$ will be established by taking advantage of the preceding analysis of the aBIP. If $G(\omega)$ is not invertible, then the Lyapunov-Stein equation (12.5) may have more than one solution $\widehat{P}$. In this case, by $\widehat{P}(x)$ we mean any nonnegative solution of $(12.5)$ corresponding to a fixed $x \in \mathbb{C}^{q \times \kappa}$.

Under assumption (1.5), the matrix pencils

$$
\widehat{G}(z)=\widehat{M}-z \widehat{N}=\left(\begin{array}{cc}
G(z) & u-z v \\
0 & \rho_{\omega}(z) I_{\kappa}
\end{array}\right)
$$

and

$$
\widehat{H}(z)=z \widehat{M}^{*}-\widehat{N}^{*}=\left(\begin{array}{cc}
H(z) & 0 \\
z u^{*}-v^{*} & \delta_{\omega}(z) I_{\kappa}
\end{array}\right)
$$

are invertible on the unit circle and therefore, the $\operatorname{aBIP}(\widehat{M}, \widehat{N}, \widehat{P}, \widehat{C})$ has a solution. This means that there exists a function $S \in \mathcal{S}^{p \times q}$ such that

$$
\left(\begin{array}{cc}
I_{p} & -S(\zeta) \\
-S(\zeta)^{*} & I_{q}
\end{array}\right) \widehat{C} \widehat{G}(\zeta)^{-1} \in\left(\begin{array}{c}
\mathbf{H}_{2}^{p \times(n+\kappa)} \\
\left(\mathbf{H}_{2}^{q \times(n+\kappa)}\right)^{\perp}
\end{array}\right)
$$

and

$$
\left[\widehat{C} \widehat{G}(\zeta)^{-1}, \widehat{C} \widehat{G}(\zeta)^{-1}\right]_{S}=\widehat{P}
$$

where $[,]_{S}$ is the matrix valued form defined via (2.1). Using the decompositions (12.4) and (12.7) we get

$$
\widehat{C} \widehat{G}^{-1}(z)=\left(C G^{-1}(z), \frac{1}{\rho_{\omega}(z)}\left\{\left(\begin{array}{c}
c_{1} \\
x
\end{array}\right)-C G^{-1}(z)(u-z v)\right\}\right),
$$

and hence that (12.8) is equivalent to (1.6) together with the condition

$$
\frac{1}{\rho_{\omega}(\zeta)}\left(-S(\zeta)^{*}, I_{q}\right)\left\{\left(\begin{array}{c}
c_{1} \\
x
\end{array}\right)-C G^{-1}(\zeta)(u-\zeta v)\right\} \in\left(\mathbf{H}_{2}^{q \times \kappa}\right)^{\perp} .
$$

Next, by Lemma 2.1, every function $S \in \mathcal{S}^{p \times q}$ satisfying the condition (12.8) (or equivalently, (1.6) and (12.11)), defines via (12.9) a nonnegative matrix $\widehat{P}$ satisfying the LyapunovStein equation (12.5). Moreover, if $S$ also satisfies (1.7), then $\widehat{P}$ is an extension of $P$, i.e., $\widehat{P}$ is of the form (12.1). Thus, Problem 12.2 reduces to finding those solutions $S$ of the $\operatorname{aBIP}(M, N, P, C)$ which also satisfy condition (12.11) and for which the value $\mathbf{D}_{r+\kappa}[\widehat{P}]$ is maximal.

Following (6.2), we define the extended function

$$
\widehat{\Delta}_{\omega}(z)=\widehat{H}(z) \widehat{P} \widehat{H}(\omega)^{*}+\rho_{\omega}(z) \widehat{C}_{1}^{*} \widehat{C}_{1} .
$$


Evaluating this function at the point $z=\omega$ and taking into account the decompositions (12.4) of $\widehat{M}, \widehat{N}$ and $\widehat{C}_{1}$ we get the matrix

$$
\begin{aligned}
\widehat{\Delta}_{\omega}(\omega) & =\left(\begin{array}{cc}
\Delta_{\omega}(\omega) & R_{1} \\
R_{1}^{*} & R_{2}
\end{array}\right) \\
& :=\left(\begin{array}{c}
H(\omega) \\
\omega u^{*}-v^{*}
\end{array}\right) P\left(H(\omega)^{*}, \bar{\omega} u-v\right)+\rho_{\omega}(\omega)\left(\begin{array}{c}
C_{1}^{*} \\
c_{1}^{*}
\end{array}\right)\left(C_{1}, c_{1}\right),
\end{aligned}
$$

which will play a central role in our considerations.

The next step is to obtain an explicit formula for the entry $q$ of $\widehat{P}$ by taking advantage of formula (12.9). Upon substituting (12.10) and the block decomposition (12.1) of $\widehat{P}$ into (12.9) we get

$$
\left[\frac{1}{\rho_{\omega}(\zeta)}\left\{\left(\begin{array}{c}
c_{1} \\
x
\end{array}\right)-C G^{-1}(\zeta)(u-\zeta v)\right\}, C G(\zeta)^{-1}\right]_{S}=q .
$$

Thus, the entry $q$ of $\widehat{P}$ is uniquely defined by $S$. An explicit expression for $q$ in terms of the initial data will be given below. Then, by (12.6), we will also obtain a formula for $d$ and thus, we shall not need the expression for $d$ in terms of the matrix form $[,]_{S}$ (which can be easily obtained from (12.9), but is not convenient for direct calculations).

As a solution of the $\operatorname{aBIP}(M, N, P, C), S$ admits a representation of the form

$$
S(z)=\Psi_{12}(z)+\Psi_{11}(z) \mathcal{E}(z)\left(I_{q}-\Psi_{21}(z) \mathcal{E}(z)\right)^{-1} \Psi_{22}(z)
$$

where the mvf's $\Psi_{j k}$ are given by (10.9)-(10.11) and (10.15) and $\mathcal{E}$ is an appropriately chosen Schur function. For a function $S$ of the form (12.14) to satisfy condition (12.11), the parameter $\mathcal{E}$ has to satisfy certain interpolation conditions, which we now establish.

Lemma 12.3 A function $S$ of the form (12.14) satisfies (12.11) if and only if the parameter $\mathcal{E}$ is subject to the bitangential interpolation condition

$$
T \mathcal{E}(\omega) Z_{1}^{*}=x^{*}-R_{1}^{*} \Delta_{\omega}^{[-1]}(\omega) C_{2}^{*},
$$

where

$$
T=c_{1}^{*} Z_{2}-\rho_{\omega}(\omega)^{-\frac{1}{2}}\left(\omega u^{*}-v^{*}\right) P^{\frac{1}{2}} Y_{2}
$$

and $R_{1}$ is defined in (12.12).

Proof: Rewrite (12.11) in the following equivalent form

$$
\frac{1}{\delta_{\omega}(\zeta)}\left\{(u-\zeta v)^{*} G(\zeta)^{-*} C^{*}-\left(c_{1}^{*}, x^{*}\right)\right\}\left(\begin{array}{c}
-S(\zeta) \\
I_{q}
\end{array}\right) \in \mathbf{H}_{2}^{k \times q} .
$$

Since $S$ is a solution of the $\operatorname{aBIP}(M, N, P, C)$ (because of (12.14)), it is easily seen that the function

$$
F(\zeta)=\left\{(u-\zeta v)^{*} G(\zeta)^{-*} C^{*}-\left(c_{1}^{*}, x^{*}\right)\right\}\left(\begin{array}{c}
-S(\zeta) \\
I_{q}
\end{array}\right)
$$


belongs $\mathbf{H}_{2}^{k \times q}$ and hence that the condition (12.17) is equivalent to the condition

$$
F(\omega)=0 .
$$

To evaluate the function $F$ at the point $\omega$ we note that in view of (10.18),

$$
(u-\zeta v)^{*} G(\zeta)^{-*} C^{*}\left(\begin{array}{c}
-\Psi_{12}(\zeta) \\
I_{q}
\end{array}\right)=\left(\zeta u^{*}-v^{*}\right) P H(\omega)^{*} \Delta_{\omega}^{[-1]}(\zeta) C_{2}^{*},
$$

whereas the first relation in (10.26) implies that

$$
(u-\zeta v)^{*} G(\zeta)^{-*} C_{1}^{*} \Psi_{11}(\zeta)=\left(\zeta u^{*}-v^{*}\right) P^{\frac{1}{2}} \Upsilon_{\omega}(\zeta) Y_{2},
$$

where $\Upsilon_{\omega}$ is the function defined in (10.27). Substituting (12.14) into (12.18) and using (12.20) and (12.21), we get

$$
\begin{aligned}
F(\zeta)= & \left(\zeta u^{*}-v^{*}\right) P H(\omega)^{*} \Delta_{\omega}^{[-1]}(\zeta) C_{2}^{*} \\
& -\left(\zeta u^{*}-v^{*}\right) P^{\frac{1}{2}} \Upsilon_{\omega}(\zeta) Y_{2} \mathcal{E}(\zeta)\left(I-\Psi_{21}(\zeta) \mathcal{E}(\zeta)\right)^{-1} \Psi_{22}(\zeta)-x^{*}+c_{1}^{*} S(\zeta) .
\end{aligned}
$$

Using (10.9)-(10.11), (10.15) and (10.27), we obtain the evaluations

$$
\begin{gathered}
\Psi_{11}(\omega)=Z_{2}, \quad \Psi_{12}(\omega)=\rho_{\omega}(\omega) C_{1} \Delta_{\omega}^{[-1]}(\omega) C_{2}^{*}, \quad \Psi_{21}(\omega)=0 \\
\Psi_{22}(\omega)=Z_{1}^{*} \quad \text { and } \quad \Upsilon_{\omega}(\omega)=\rho_{\omega}(\omega)^{-\frac{1}{2}} I_{n}
\end{gathered}
$$

which, upon being substituted into (12.22) and (12.14), lead to

$$
\begin{aligned}
F(\omega)= & \left(\omega u^{*}-v^{*}\right) P H(\omega)^{*} \Delta_{\omega}^{[-1]}(\omega) C_{2}^{*} \\
& -\rho_{\omega}(\omega)^{-\frac{1}{2}}\left(\omega u^{*}-v^{*}\right) P^{\frac{1}{2}} Y_{2} \mathcal{E}(\omega) Z_{1}^{*}-x^{*}+c_{1}^{*} S(\omega)
\end{aligned}
$$

and

$$
S(\omega)=\rho_{\omega}(\omega) C_{1} \Delta_{\omega}^{[-1]}(\omega) C_{2}^{*}+Z_{2} \mathcal{E}(\omega) Z_{1}^{*}
$$

respectively. Substituting the two latter evaluations into (12.19) we get

$$
\begin{aligned}
& \left(c_{1}^{*} Z_{2}-\rho_{\omega}(\omega)^{-\frac{1}{2}}\left(\omega u^{*}-v^{*}\right) P^{\frac{1}{2}} Y_{2}\right) \mathcal{E}(\omega) Z_{1}^{*} \\
& =x^{*}-\left\{\left(\omega u^{*}-v^{*}\right) P H(\omega)^{*}+\rho_{\omega}(\omega) c_{1}^{*} C_{1}\right\} \Delta_{\omega}^{[-1]}(\omega) C_{2}^{*} .
\end{aligned}
$$

It is easily seen from (12.12) and (12.16) that the last equality coincides with (12.15).

Note that the proof of the last lemma is much simpler if $H(\omega)$ is invertible, because then

$$
F(\zeta)=\left\{\left(\zeta u^{*}-v^{*}\right) H(\zeta)^{-1} C^{*}-\left(c_{1}^{*}, x^{*}\right)\right\}\left(\begin{array}{c}
-S(\zeta) \\
I_{q}
\end{array}\right)
$$

and hence, by (12.24),

$$
\begin{aligned}
F(\omega)= & \left(c_{1}^{*}-\left(\omega u^{*}-v^{*}\right) H(\omega)^{-1} C_{1}^{*}\right)\left(\rho_{\omega}(\omega) C_{1} \Delta_{\omega}^{[-1]}(\omega) C_{2}^{*}+Z_{2} \mathcal{E}(\omega) Z_{1}^{*}\right) \\
& -x^{*}+\left(\omega u^{*}-v^{*}\right) H(\omega)^{-1} C_{2}^{*} .
\end{aligned}
$$


Thus $F(\omega)=0$ if and only if

$$
\begin{array}{r}
\left(c_{1}^{*}-\left(\omega u^{*}-v^{*}\right) H(\omega)^{-1} C_{1}^{*}\right) Z_{2} \mathcal{E}(\omega) Z_{1}^{*}=x^{*}-\rho_{\omega}(\omega) c_{1}^{*} C_{1} \Delta_{\omega}^{[-1]}(\omega) C_{2}^{*} \\
-\left(\omega u^{*}-v^{*}\right) H(\omega)^{-1}\left\{I-\rho_{\omega}(\omega) C_{1}^{*} C_{1} \Delta_{\omega}^{[-1]}(\omega)\right\} C_{2}^{*} .
\end{array}
$$

This condition is equivalent to (12.15), since

$$
\left\{I-\rho_{\omega}(\omega) C_{1}^{*} C_{1} \Delta_{\omega}^{[-1]}(\omega)\right\} C_{2}^{*}=H(\omega) P H(\omega)^{*} \Delta_{\omega}^{[-1]}(\omega) C_{2}^{*}
$$

(by (10.22) and (10.20)) and

$$
\left(c_{1}^{*}-\left(\omega u^{*}-v^{*}\right) H(\omega)^{-1} C_{1}^{*}\right) Z_{2}=c_{1}^{*} Z_{2}-\rho_{\omega}(\omega)^{-\frac{1}{2}}\left(\omega u^{*}-v^{*}\right) P^{\frac{1}{2}} Y_{2}=T
$$

(by the first relation in (10.28)).

Lemma 12.4 Let $S$ be of the form (12.14) for some choice of the Schur function $\mathcal{E}$, let $q$ be defined via (12.13) and let $R_{1}$ and $T$ be given by (12.12) and (12.16), respectively. Then

$$
q=\frac{1}{\rho_{\omega}(\omega)} P G(\omega) \Delta_{\omega}^{[-1]}(\omega) R_{1}-\frac{1}{\rho_{\omega}(\omega)} P(u-\omega v)+\rho_{\omega}(\omega)^{-\frac{1}{2}} P^{\frac{1}{2}} Y_{1} \mathcal{E}(\omega)^{*} T^{*} .
$$

Proof: By Remark 6.7, the rational function $\Delta_{\omega}^{[-1]}(z)$ is analytic in $\overline{\mathbb{D}}$ and thus, it belongs to $\mathbf{H}_{2}^{n \times n}$. In view of (10.17) and the second relation in (10.26),

$$
\begin{aligned}
\left(I_{p},-S(z)\right) C G^{-1}(z)= & \left(I_{p},-\Psi_{12}(z)\right) C G^{-1}(z) \\
& -\Psi_{11}(z) \mathcal{E}(z)\left(I_{q}-\Psi_{21}(z) \mathcal{E}(z)\right)^{-1} \Psi_{22}(z) C_{2} G^{-1}(z) \\
= & C_{1} \Delta_{\omega}^{[-1]}(z) G(\omega)^{*} P \\
& +\Psi_{11}(z) \mathcal{E}(z)\left(I_{q}-\Psi_{21}(z) \mathcal{E}(z)\right)^{-1} Y_{1}^{*} \Upsilon_{\omega}(z) P^{\frac{1}{2}} .
\end{aligned}
$$

Thus, by (12.23),

$$
\left(\left(I_{p},-S\right) C G^{-1}\right)(\omega)=C_{1} \Delta_{\omega}^{[-1]}(\omega) G(\omega)^{*} P+\rho_{\omega}(\omega)^{-\frac{1}{2}} Z_{2} \mathcal{E}(\omega) Y_{1}^{*} P^{\frac{1}{2}} .
$$

Moreover, since $S$ is a solution of the $\operatorname{aBIP}(M, N, P, C)$, condition (1.6) is in force. Therefore,

$$
\begin{aligned}
(1) & =\left[\frac{1}{\rho_{\omega}(\zeta)}\left(\begin{array}{c}
c_{1} \\
x
\end{array}\right), C G(\zeta)^{-1}\right]_{S} \\
& =\left\langle\frac{1}{\rho_{\omega}(\zeta)}\left(\begin{array}{c}
c_{1} \\
x
\end{array}\right), \quad\left(\begin{array}{cc}
I_{p} & -S(\zeta) \\
-S(\zeta)^{*} & I_{q}
\end{array}\right) C G(\zeta)^{-1}\right\rangle \\
& =\left\langle\frac{c_{1}}{\rho_{\omega}(\zeta)}, \quad\left(I_{p},-S(\zeta)\right) C G(\zeta)^{-1}\right\rangle \\
& =P G(\omega) \Delta_{\omega}^{[-1]}(\omega) C_{1}^{*} c_{1}+\rho_{\omega}(\omega)^{-\frac{1}{2}} P^{\frac{1}{2}} Y_{1} \mathcal{E}(\omega)^{*} Z_{2}^{*} c_{1} .
\end{aligned}
$$


Next, upon taking advantage of (10.19), it is readily checked that

$$
\begin{aligned}
2= & {\left[\frac{1}{\rho_{\omega}(\zeta)} C G(\zeta)^{-1}(u-\zeta v), C G(\zeta)^{-1}\right]_{\Psi_{12}} } \\
= & \left\langle\frac{1}{\rho_{\omega}(\zeta)} C G(\zeta)^{-1}(u-\zeta v),\left(\begin{array}{cc}
I_{p} & -\Psi_{12}(\zeta) \\
-\Psi_{12}(\zeta)^{*} & I_{q}
\end{array}\right) C G(\zeta)^{-1}\right\rangle \\
= & \rho_{\omega}(\omega) P\left\langle\frac{1}{\rho_{\omega}^{2}(\zeta)}(u-\zeta v), \frac{I_{n}}{\rho_{\omega}(\zeta)}\right\rangle \\
& -P G(\omega)\left\langle\frac{I_{n}}{\rho_{\omega}(\zeta)}, \frac{1}{\rho_{\omega}(\zeta)}\left(\zeta u^{*}-v^{*}\right) P H(\omega)^{*} \Delta_{\omega}^{[-1]}(\zeta)\right\rangle \\
& -P H(\omega)^{*}\left\langle\frac{1}{\rho_{\omega}(\zeta)} \Delta_{\omega}^{[-1]}(\zeta) G(\omega)^{*} P(u-\zeta v), \frac{I_{n}}{\delta_{\omega}(\zeta)}\right\rangle \\
= & \frac{1}{\rho_{\omega}(\omega)} P(u-\omega v)-\frac{1}{\rho_{\omega}(\omega)} P G(\omega) \Delta_{\omega}^{[-1]}(\omega) H(\omega) P(\bar{\omega} u-v),
\end{aligned}
$$

since $\rho_{\omega}(z)^{-1} I_{n}$ is the reproducing kernel for $\mathbf{H}_{2}^{n}$ and $\delta_{\omega}(z)^{-1} u$ is orthogonal to $\mathbf{H}_{2}^{n}$ for every vector $u \in \mathbb{C}^{n}$.

Now set

$$
S_{\varepsilon}(z)=\Psi_{11}(z) \mathcal{E}(z)\left(I_{q}-\Psi_{21}(z) \mathcal{E}(z)\right)^{-1} \Psi_{22}(z)
$$

so that

$$
S(z)=\Psi_{12}(z)+S_{\varepsilon}(z)
$$

It is easily seen that

$$
\left\langle\frac{1}{\rho_{\omega}(\zeta)} C G(\zeta)^{-1}(u-\zeta v), \quad\left(\begin{array}{cc}
0 & S_{\varepsilon}(\zeta) \\
S_{\varepsilon}(\zeta)^{*} & 0
\end{array}\right) C G(\zeta)^{-1}\right\rangle=\left\langle B_{1}, \quad I_{n}\right\rangle+\left\langle\frac{I_{n}}{\rho_{\omega}}, \quad B_{2}\right\rangle
$$

where

$$
B_{1}(\zeta):=\frac{1}{\rho_{\omega}(\zeta)} G(\zeta)^{-*} C_{1}^{*} S_{\varepsilon}(\zeta) C_{2} G(\zeta)^{-1}(u-\zeta v)
$$

and

$$
B_{2}(\zeta):=\left(u^{*}-\bar{\zeta} v^{*}\right) G(\zeta)^{-*} C_{1}^{*} S_{\varepsilon}(\zeta) C_{2} G(\zeta)^{-1}
$$

In view of (10.26),

$$
G(\zeta)^{-*} C_{1}^{*} S_{\varepsilon}(\zeta) C_{2} G(\zeta)^{-1}=-\zeta P^{\frac{1}{2}} \Upsilon_{\omega}(\zeta) Y_{2} \mathcal{E}(\zeta)\left(I_{q}-\Psi_{21}(\zeta) \mathcal{E}(\zeta)\right)^{-1} Y_{1}^{*} \Upsilon_{\omega}(\zeta) P^{\frac{1}{2}}
$$

which implies in particular, that $B_{1}$ belongs to $\zeta \mathbf{H}_{2}^{n \times \kappa}$ whereas $B_{2}$ belongs to $\mathbf{H}_{2}^{n \times \kappa}$. Therefore, the first scalar product on the right hand side of (12.26) is equal to zero, whereas the second is equal to

$$
B_{2}(\omega)^{*}=\frac{1}{\rho_{\omega}(\omega)} P^{\frac{1}{2}} Y_{1} \mathcal{E}(\omega)^{*} Y_{2}^{*} P^{\frac{1}{2}}(v-\bar{\omega} u)
$$


Finally, upon substituting these evaluations into the definition (12.13) of $q$, we obtain

$$
\begin{aligned}
q= & (1)-(2)+B_{2}(\omega)^{*} \\
= & P G(\omega) \Delta_{\omega}^{[-1]}(\omega)\left\{C_{1}^{*} c_{1}+\frac{1}{\rho_{\omega}(\omega)} H(\omega) P(\bar{\omega} u-v)\right\} \\
& +\rho_{\omega}(\omega)^{-\frac{1}{2}} P^{\frac{1}{2}} Y_{1} \mathcal{E}(\omega)^{*}\left\{Z_{2}^{*} c_{1}+\rho_{\omega}(\omega)^{-\frac{1}{2}} Y_{2}^{*} P^{\frac{1}{2}}(v-\bar{\omega} u)\right\}-\frac{1}{\rho_{\omega}(\omega)} P(u-\omega v) .
\end{aligned}
$$

The last formula coincides with (12.25) in view of the definitions (12.12) and (12.16) of $R_{1}$ and $T$.

It was mentioned above that $q$ is not uniquely defined by $x$. This fact follows from the last lemma. Indeed, by $(12.15), x$ defines the matrix $T \mathcal{E}(\omega) Z_{1}^{*}$, but not $T \mathcal{E}(\omega) Y_{1}^{*}$, which appears in the expression (12.25) for $q$. However, if $P G(\omega)$ is invertible, then the matrix $Z_{1}$ is square and invertible. In this case (which was considered in [15]), $x$ defines the matrix $T \mathcal{E}(\omega)$ by (12.15) and thus, also $q$, via formula (12.25).

Now we shall establish the extremal value of $d-q^{*} P^{[-1]} q$, the Schur complement of $P$ in $\widehat{P}$. Using (12.6) together with (12.25) we get by straightforward calculation

$$
d-q^{*} P^{[-1]} q=\rho_{\omega}(\omega)^{-2}\left\{R_{2}-\rho_{\omega}(\omega) x^{*} x-\mathbf{Y}^{*} \mathbf{Y}\right\},
$$

where

$$
\mathbf{Y}=\rho_{\omega}(\omega)^{\frac{1}{2}} Y_{1} \mathcal{E}(\omega)^{*} T^{*}+P^{\frac{1}{2}} G(\omega) \Delta_{\omega}^{[-1]}(\omega) R_{1}
$$

and $R_{1}$ and $R_{2}$ are defined in (12.12). By (12.15),

$$
x=Z_{1} \mathcal{E}(\omega)^{*} T^{*}+C_{2} \Delta_{\omega}^{[-1]}(\omega) R_{1}
$$

and therefore,

$$
\begin{aligned}
\left(\begin{array}{c}
\mathbf{Y} \\
\rho_{\omega}(\omega)^{\frac{1}{2}} x
\end{array}\right) & =\rho_{\omega}(\omega)^{\frac{1}{2}}\left(\begin{array}{c}
Y_{1} \\
Z_{1}
\end{array}\right) \mathcal{E}(\omega)^{*} T^{*}+\left(\begin{array}{c}
P^{\frac{1}{2}} G(\omega) \\
\rho_{\omega}(\omega)^{\frac{1}{2}} C_{2}
\end{array}\right) \Delta_{\omega}^{[-1]}(\omega) R_{1} \\
& =\rho_{\omega}(\omega)^{\frac{1}{2}}\left(\begin{array}{c}
Y_{1} \\
Z_{1}
\end{array}\right) \mathcal{E}(\omega)^{*} T^{*}+W_{1} \Delta_{\omega}^{[-1]}(\omega) R_{1} .
\end{aligned}
$$

Therefore, since $\left(\begin{array}{c}Y_{1} \\ Z_{1}\end{array}\right)$ is an isometric column of $W_{1}^{\perp}$ and $W_{1}^{*} W_{1}^{\perp}=0$,

$$
\rho_{\omega}(\omega) x^{*} x+\mathbf{Y}^{*} \mathbf{Y}=\rho_{\omega}(\omega) T \mathcal{E}(\omega) \mathcal{E}(\omega)^{*} T^{*}+R_{1}^{*} \Delta_{\omega}^{[-1]}(\omega) R_{1} .
$$

Consequently,

$$
d-q^{*} P^{[-1]} q=\rho_{\omega}(\omega)^{-2}\left\{R_{2}-R_{1}^{*} \Delta_{\omega}^{[-1]}(\omega) R_{1}-\rho_{\omega}(\omega) T \mathcal{E}(\omega) \mathcal{E}(\omega)^{*} T^{*}\right\},
$$

and thus,

$$
d-q^{*} P^{[-1]} q \leq \rho_{\omega}(\omega)^{-2}\left\{R_{2}-R_{1}^{*} \Delta_{\omega}^{[-1]}(\omega) R_{1}\right\}
$$


with equality if and only if

$$
T \mathcal{E}(\omega)=0 .
$$

It follows from (12.15) that for every such parameter, the corresponding extremal matrix $x$ is uniquely defined and is equal to

$$
x=C_{2} \Delta_{\omega}^{[-1]}(\omega) R_{1} .
$$

Upon substituting (12.28) and (12.29) into (12.25) and (12.6) we obtain explicit formulas for the block entries $q$ and $d$ of the extremal matrix $\widehat{P}(x)$ :

$$
\begin{aligned}
q=\rho_{\omega}(\omega)^{-1} P\{ & \left.G(\omega) \Delta_{\omega}^{[-1]}(\omega) R_{1}-(u-\omega v)\right\} \\
d=\rho_{\omega}(\omega)^{-2}\{ & R_{2}+(u-\omega v)^{*} P(u-\omega v)-\rho_{\omega}(\omega) R_{1}^{*} \Delta_{\omega}^{[-1]}(\omega) G(\omega)^{*} P(u-\omega v) \\
& \quad \rho_{\omega}(\omega)(u-\omega v)^{*} P G(\omega) \Delta_{\omega}^{[-1]}(\omega) R_{1} \\
& \left.\quad-\rho_{\omega}(\omega) R_{1}^{*} \Delta_{\omega}^{[-1]}(\omega) C_{2}^{*} C_{2} \Delta_{\omega}^{[-1]}(\omega) R_{1}\right\} .
\end{aligned}
$$

We summarize the results obtained above in the following theorem.

Theorem 12.5 Let $\widehat{M}, \widehat{N}, \widehat{C}_{1}$ and $\widehat{C}_{2}$ be defined as in (12.4), where all entries except for the entry $x$ of $\widehat{C}_{2}$ are given. Let $Y_{j}, Z_{j}, R_{j}$ and $T$ be given by (10.7), (12.12) and (12.16), respectively. Then:

1. There exists a nonnegative solution $\widehat{P}=\widehat{P}(x)$ of the form (12.1) to the Lyapunov-Stein equation (12.5) if and only if

$$
x=Z_{1} \mathbf{S} T^{*}+C_{2} \Delta_{\omega}^{[-1]}(\omega) R_{1}
$$

for some contractive matrix $\mathbf{S}$, i.e., if and only if $x$ belongs to the matrix ball with center $C_{2} \Delta_{\omega}^{[-1]}(\omega) R_{1}$, left semi-radius $\left(Z_{1} Z_{1}^{*}\right)^{\frac{1}{2}}$ and right semi-radius $\left(T T^{*}\right)^{\frac{1}{2}}$.

2. The Schur complement of $P$ in $\widehat{P}(x)$ is equal to

$$
d-q^{*} P^{[-1]} q=E-\rho_{\omega}(\omega)^{-2} T \mathbf{S}^{*} \mathbf{S} T^{*},
$$

where

$$
E=\rho_{\omega}(\omega)^{-2}\left\{R_{2}-R_{1}^{*} \Delta_{\omega}^{[-1]}(\omega) R_{1}\right\}
$$

is proportional to the Schur complement of $\Delta_{\omega}(\omega)$ in the matrix $\widehat{\Delta}_{\omega}(\omega)$ given by (12.12).

3. $E \geq 0$ for every choice of $\kappa \geq 1$. If $\operatorname{det} E=0$, then $\mathbf{D}_{r+\kappa}[\widehat{P}(x)]=0$ for every choice of the matrix $x$.

4. If $E>0$, then $\mathbf{D}_{r+\kappa}[\widehat{P}(x)] \leq \mathbf{D}_{r}[P] \times \operatorname{det} E$ with equality if and only if formulas (12.29)-(12.31) prevail. 
If $P>0$ and $G(\omega)$ is invertible, then Theorem 12.5 reduces to Theorem 2.1 of [15]. In this case $r=n$, all the pseudoinverses in the statement of Theorem 12.5 are ordinary inverses and the formulas furnished for $E$ and $x$ are the same in both theorems. Moreover, $\mathbf{D}_{r}(P)$ and $\mathbf{D}_{r+\kappa}(\widehat{P})$ in item 4 are both ordinary determinants.

Finally, we complete this section with a short discussion of another variant of the extension problem 12.2: let $M, N \in \mathbb{C}^{n \times n}$ satisfy the condition (1.5), let $P \in \mathbb{C}^{n \times n}$ be a positive semidefinite solution of the Lyapunov-Stein equation (1.10) with rank $P=r \leq n$ as above, but now let

$$
\begin{aligned}
& \widehat{M}=\left(\begin{array}{cc}
M & u \\
0 & \bar{\omega} I_{\kappa}
\end{array}\right), \quad \widehat{N}=\left(\begin{array}{cc}
N & v \\
0 & I_{\kappa}
\end{array}\right) \quad(|\omega|<1), \\
& \widehat{C}_{1}=\left(C_{1}, y\right) \quad \text { and } \quad \widehat{C}_{1}=\left(C_{2}, c_{2}\right) \quad\left(c_{1} \in \mathbb{C}^{p \times \kappa}, x \in \mathbb{C}^{q \times \kappa}\right) .
\end{aligned}
$$

Problem 12.6 Find those positive semidefinite solutions $\widehat{P}=\widehat{P}(y)$ of the Lyapunov-Stein equation (12.5) which attain the maximum value of $\mathbf{D}_{r+\kappa}(\widehat{P})$.

This problem can be solved by using the same methods which were applied to solve Problem 12.2. The main role now is played by the matrix

$$
\begin{aligned}
\widehat{\Delta}_{\omega}(\omega) & =\left(\begin{array}{cc}
\Delta_{\omega}(\omega) & \widetilde{R}_{1} \\
\widetilde{R}_{1}^{*} & \widetilde{R}_{2}
\end{array}\right) \\
& :=\left(\begin{array}{c}
G(\omega)^{*} \\
u^{*}-\bar{\omega} v^{*}
\end{array}\right) P(G(\omega), u-\omega v)+\rho_{\omega}(\omega)\left(\begin{array}{c}
C_{2}^{*} \\
c_{2}^{*}
\end{array}\right)\left(C_{2}, c_{2}\right),
\end{aligned}
$$

which is the value of the function

$$
\widehat{\Delta}_{\omega}(z)=\left(\widehat{M}^{*}-\bar{\omega} \widehat{N}^{*}\right) \widehat{P}(\widehat{M}-z \widehat{N})+\rho_{\omega}(z) \widehat{C}_{2}^{*} \widehat{C}_{2}
$$

at the point $z=\omega$.

Theorem 12.7 Let $\widehat{M}, \widehat{N}, \widehat{C}_{1}$ and $\widehat{C}_{2}$ be defined as in (12.32), where all entries except for the entry $y$ of $\widehat{C}_{1}$ are given. Let $Y_{j}, Z_{j}$ and $\widetilde{R}_{j}$ be given by (10.7) and (12.33), respectively. Then:

1. There exists a nonnegative solution $\widehat{P}=\widehat{P}(y)$ of the form (12.1) to the Lyapunov-Stein equation (12.5) if and only if

$$
y=Z_{2} \mathbf{S} \widetilde{T}^{*}+C_{1} \Delta_{\omega}^{[-1]}(\omega) \widetilde{R}_{1}
$$

for some contractive matrix $\mathbf{S}$, where

$$
\widetilde{T}=c_{2}^{*} Z_{1}+\rho_{\omega}(\omega)^{-\frac{1}{2}}(u-\omega v)^{*} P^{\frac{1}{2}} Y_{1} .
$$

2. The Schur complement of $P$ in $\widehat{P}(y)$,

$$
d-q^{*} P^{[-1]} q=\widetilde{E}-\rho_{\omega}(\omega)^{-2} \widetilde{T} \mathbf{S}^{*} \mathbf{S} \widetilde{T}^{*},
$$

where

$$
\widetilde{E}=\rho_{\omega}(\omega)^{-2}\left\{\widetilde{R}_{2}-\widetilde{R}_{1}^{*} \Delta_{\omega}^{[-1]}(\omega) \widetilde{R}_{1}\right\}
$$

is proportional to the Schur complement of $\Delta_{\omega}(\omega)$ in the matrix $\widehat{\Delta}_{\omega}(\omega)$ given by $(12.33)$. 
3. $\widetilde{E} \geq 0$ for every choice $\kappa \geq 1$. If $\operatorname{det} \widetilde{E}=0$, then $\mathbf{D}_{r+\kappa}[\widehat{P}(y)]=0$ for every choice of the matrix $y$.

4. If $\widetilde{E}>0$, then $\mathbf{D}_{r+\kappa}[\widehat{P}(y)] \leq \mathbf{D}_{r}[P] \times \operatorname{det} \widetilde{E}$ with equality if and only if

$$
\begin{aligned}
& y=C_{1} \Delta_{\omega}^{[-1]}(\omega) \widetilde{R}_{1} \\
& q=-\rho_{\omega}(\omega)^{-1} P\left\{H(\omega)^{*} \Delta_{\omega}^{[-1]}(\omega) \widetilde{R}_{1}+(v-\bar{\omega} u)\right\} \\
& \text { and } \\
& d=\rho_{\omega}(\omega)^{-2}\left\{\widetilde{R}_{2}+(v-\bar{\omega} u)^{*} P(v-\bar{\omega} u)+\rho_{\omega}(\omega) \widetilde{R}_{1}^{*} \Delta_{\omega}^{[-1]}(\omega) H(\omega) P(v-\bar{\omega} u)\right. \\
& +\rho_{\omega}(\omega)(v-\bar{\omega} u)^{*} P H(\omega)^{*} \Delta_{\omega}^{[-1]}(\omega) \widetilde{R}_{1} \\
& \left.-\rho_{\omega}(\omega) \widetilde{R}_{1}^{*} \Delta_{\omega}^{[-1]}(\omega) C_{1}^{*} C_{1} \Delta_{\omega}^{[-1]}(\omega) \widetilde{R}_{1}\right\} \text {. }
\end{aligned}
$$

If $H(\omega)$ is invertible, then the entries $q$ and $d$ of $\widehat{P}$ are uniquely defined by $y$. In this case, the matrix $\widehat{P}(y)$ which maximizes the value of $\mathbf{D}_{r+\kappa}[\widehat{P}(y)]$ corresponds to the choice (12.35) of $y$. Under the additional assumption $P>0$, this situation is described in $[15$, Theorem 2.2]. Note also that in this case (in contrast to the degenerate situation), the set (12.34) of all admissible matrices $y$ is the full rank matrix ball (i.e., with strictly positive semi-radii). If $H(\omega)$ is not invertible, there are, in general, many matrices $\widehat{P}(y)$ corresponding via (12.5) to the same $y$. There is, however, only one (with entries $q$ and $d$ given by (12.36) and (12.37), respectively), which maximizes $\mathbf{D}_{r+\kappa}[\widehat{P}(y)]$ and this matrix corresponds to the $y$ defined in $(12.35)$.

\section{Other domains}

For ease of exposition we have focused in this paper on interpolation problems in the unit disc. However, the notation has been chosen so that most of the results discussed above can easily be transferred to the open right half plane, or the open upper half plane or even more general regions by introducing a few auxiliary symbols, much as in [3]-[?]. To illustrate this, let $a, b$ be a pair of functions which are analytic and have no common zeros in an open connected subset $\Omega \subseteq \mathbb{C}$, let

$$
\rho_{\omega}(z)=a(z) a(\omega)^{*}-b(z) b(\omega)^{*} \quad \text { and } \quad \delta_{\omega}(z)=b(z) a(\omega)-a(z) b(\omega)
$$

and let the subsets

$$
\Omega_{+}=\left\{z \in \Omega: \rho_{z}(z)>0\right\} \quad \text { and } \quad \Omega_{-}=\left\{z \in \Omega: \rho_{z}(z)<0\right\}
$$

be nonempty. Then there exists a point $\mu$ such that $|a(\mu)|=|b(\mu)| \neq 0$ (see e.g., [4]) and hence, the subset

$$
\Omega_{0}=\left\{z \in \Omega: \rho_{z}(z)=0\right\}
$$

is non-empty.

The kernel $\rho_{\omega}(z)^{-1}$ is nonnegative in $\Omega_{+}$. This means that for every choice of integer $\kappa \in$ 
$\mathbb{N}$ and of points $\omega_{1}, \ldots, \omega_{\kappa}$ in $\Omega_{+}$, the $\kappa \times \kappa$ Hermitian matrix with $i j$-entry $\rho_{\omega_{i}}\left(\omega_{j}\right)^{-1}$ is nonnegative. Therefore, there exists a reproducing kernel Hilbert space $\mathbf{H}_{\rho}$ with reproducing kernel $\rho_{\omega}(z)^{-1}$. The following direct characterization of the space $\mathbf{H}_{\rho}$ is given in [4, p. 127].

Theorem 13.1 The space $\mathbf{H}_{\rho}$ consists of functions $\tilde{f}$ which are analytic on $\Omega_{+}$and admit a representation of the form

$$
\tilde{f}(z)=\frac{1}{a(z)} \sum_{j=0}^{\infty} \tilde{f}_{j} \sigma(z)^{j}, \quad \text { where } \quad \sigma(z)=\frac{b(z)}{a(z)},
$$

and with

$$
\|\tilde{f}\|_{\mathbf{H}_{\rho}}^{2}=\sum_{j=0}^{\infty}\left|\tilde{f}_{j}\right|^{2}<\infty
$$

We refer to the papers [3], [4] and [?] for further properties of the spaces $\mathbf{H}_{\rho}$ and for various examples of $\rho$. The case of the unit disc considered above, corresponds to the choice $a(z)=1$ and $b(z)=z$. Then $\Omega_{+}=\mathbb{D}, \Omega_{0}=\mathbb{\Gamma}, \rho_{\omega}(z)$ and $\delta_{\omega}(z)$ are given in (1.16) and $\mathbf{H}_{\rho}$ is the Hardy space $\mathbf{H}_{2}$ of the unit disc. The following three cases are of interest:
(1) $\left\{\begin{array}{l}a(z)=\sqrt{\pi}(1-i z) \\ b(z)=\sqrt{\pi}(1+i z)\end{array}\right.$
$\left\{\begin{array}{l}a(z)=\sqrt{\pi}(1+z) \\ b(z)=\sqrt{\pi}(1-z)\end{array}\right.$
(3) $\left\{\begin{array}{l}a(z)=\sqrt{\pi}\left\{z+i\left(z^{2}+1\right)\right\} \\ b(z)=\sqrt{\pi}\left\{z-i\left(z^{2}+1\right)\right\}\end{array}\right.$

In the first case, $\rho_{\omega}(z)=-2 \pi i(z-\bar{\omega}), \Omega_{+}=\mathbb{C}_{+}$(the open upper half plane) and $\Omega_{0}=\mathbb{R}$. In the second case, $\rho_{\omega}(z)=2 \pi(z+\bar{\omega}), \Omega_{+}$is the right half plane and $\Omega_{0}$ is the imaginary axis. In the third case, $\rho_{\omega}(z)=-2 \pi i(z-\bar{\omega})(1-z \bar{\omega}), \Omega_{0}=\mathbb{R} \cup \mathbb{T}$ and $\Omega_{+}=\left(\mathbb{D} \cap \mathbb{C}_{+}\right) \cup\left(\mathbb{E} \cap \mathbb{C}_{-}\right)$ (where $\mathbb{E}=\mathbb{C} \backslash \overline{\mathbb{D}}$ and $\mathbb{C}_{-}$denotes the open lower half plane). Note that, in this case, $\Omega_{+}$is not connected.

We define $\mathcal{S}_{\rho}^{p \times q}$ to be the set of all $\mathbb{C}^{p \times q}$-valued functions $\widetilde{S}$ analytic on $\Omega_{+}$for which the kernel

$$
k(z, \omega)=\frac{I_{p}-\widetilde{S}(z) \widetilde{S}(\omega)^{*}}{\rho_{\omega}(z)}
$$

is nonnegative on $\Omega_{+}$. This is the same as to say that the operator of multiplication by $\widetilde{S}$ is a contraction from $\mathbf{H}_{\rho}^{q}$ into $\mathbf{H}_{\rho}^{p}$ (see [3]). It follows from the nonnegativity of the kernel $k$ that $\widetilde{S}$ of the class $\mathcal{S}_{\rho}^{p \times q}$ takes contractive values. However, although the opposite implication is valid in the classical settings (when $a(z)$ and $b(z)$ are polynomials of degree less than or equal to one), it is not always in force. For example, if $a(z)=1$ and $b(z)=z^{2}$, then $\Omega_{+}=\mathbb{D}$, but not every analytic contraction $\widetilde{S}$ in $\mathbb{D}$ induces a nonnegative kernel $\frac{I_{p}-\widetilde{S}(z) \widetilde{S}(\omega)^{*}}{1-z^{2} \bar{\omega}^{2}}$.

The following lemma (for a proof see [2, Theorem 2.4]) is an analogue of Theorem 13.1 for Schur functions.

Lemma 13.2 A function $\widetilde{S}$ belongs to $\mathcal{S}_{\rho}^{p \times q}$ if and only if it is of the form

$$
\widetilde{S}(z)=S(\sigma(z)), \quad \text { where } \quad \sigma(z)=\frac{b(z)}{a(z)} \quad \text { and } \quad S \in \mathcal{S}^{p \times q} .
$$


The classical Nevanlinna-Pick and Carathéodory-Fejér problems for functions $S \in \mathcal{S}_{\rho}^{p \times q}$ were studied in [1] and [3]. To formulate the general aBIP for functions $S \in \mathcal{S}_{\rho}^{p \times q}$ we need to introduce $\rho$-analogues of the spaces $L_{2}$ and $\mathbf{H}_{2}^{\perp}$.

We shall let $L_{\rho}^{k}$ denote the set of $k \times 1$ mvf's which are defined almost everywhere on $\Omega_{0}$ and can be expressed in the form

$$
\tilde{f}(\xi)=\frac{1}{a(\xi)} \sum_{j=-\infty}^{\infty} \tilde{f}_{j} \sigma(\xi)^{j} \quad\left(\xi \in \Omega_{0}\right)
$$

with $\tilde{f}_{j} \in \mathbb{C}^{k}$ and

$$
\|\tilde{f}\|_{L_{\rho}}^{2}:=\sum_{j=-\infty}^{\infty}\left\|\tilde{f}_{j}\right\|^{2}<\infty
$$

Following (13.4), we say that a function $\tilde{f} \in L_{\rho}^{k}$ belongs to $\mathbf{H}_{\rho}^{k}\left(\operatorname{respectively,~}\left(\mathbf{H}_{\rho}^{k}\right)^{\perp}\right)$ if $\tilde{f}_{j}=0$ for all $j<0$ (respectively, $j \geq 0$ ) in the expansion (13.6). The following lemma reformulates the characterization of the above spaces in a form which is more suitable for the present framework.

Lemma 13.3 A function $\widetilde{f}$ belongs to $L_{\rho}^{k}$ if and only if it can be represented in the form

$$
\widetilde{f}(\xi)=\frac{1}{a(\xi)} f(\sigma(\xi)) \quad \text { for } \quad f \in L_{2}^{k}(\mathbb{T})
$$

The function $f$ is uniquely defined and $\|\widetilde{f}\|_{L_{\rho}^{k}}=\|f\|_{L_{2}^{k}(\mathbb{T})}$. Moreover, $\tilde{f} \in \mathbf{H}_{\rho}^{k}$ (respectively, $\tilde{f} \in\left(\mathbf{H}_{\rho}^{k}\right)^{\perp}$ ) if and only $f \in \mathbf{H}_{2}^{k}$ (respectively, $f \in\left(\mathbf{H}_{2}^{k}\right)^{\perp}$ ) in the representation (13.7).

Much as in the case of the unit disc, we shall let $\mathbf{H}_{\rho}^{p \times q}$ and $\left(\mathbf{H}_{\rho}^{p \times q}\right)^{\perp}$ denote the spaces of $p \times q$ matrices with entries in $\mathbf{H}_{\rho}$ and $\mathbf{H}_{\rho}^{\perp}$, respectively.

Let $a$ and $b$ be given analytic functions and let $\Omega_{0}$ be the set defined in (13.3). We assume that

$$
M, N, P \in \mathbb{C}^{n \times n} \text { and } C \in \mathbb{C}^{(p+q) \times n}
$$

is a given set of matrices and let $\operatorname{aBIP}(M, N, P, C, \rho)$ denote the following interpolation problem under the assumption that the mvf

$$
\widetilde{G}(z)=a(z) M-b(z) N
$$

is invertible at every point on $\Omega_{0}$ :

$$
\operatorname{det} \widetilde{G}(\xi) \neq 0 \quad \text { for } \quad \xi \in \Omega_{0} \text {. }
$$

Find all the functions $\widetilde{S} \in \mathcal{S}_{\rho}^{p \times q}$ such that

$$
\left(\begin{array}{cc}
I_{p} & -\widetilde{S}(\xi) \\
-\widetilde{S}(\xi)^{*} & I_{q}
\end{array}\right) C \widetilde{G}^{-1}(\xi) \in\left(\begin{array}{c}
\mathbf{H}_{\rho}^{p \times n} \\
\left(\mathbf{H}_{\rho}^{q \times n}\right)^{\perp}
\end{array}\right)
$$


and

$$
\left\langle\left(\begin{array}{cc}
I_{p} & -\widetilde{S}(\xi) \\
-\widetilde{S}(\xi)^{*} & I_{q}
\end{array}\right) C \widetilde{G}^{-1}(\xi) y, C \widetilde{G}^{-1}(\xi) x\right\rangle_{L_{\rho}^{p+q}}=x^{*} P y \quad\left(\forall x, y \in \mathbb{C}^{n}\right) .
$$

The next lemma allows us to reduce the $\operatorname{aBIP}(M, N, P, C, \rho)$ to the $\operatorname{aBIP}(M, N, P, C)$ in the unit disc which was considered above.

Lemma 13.4 A function $\widetilde{S}$ is a solution of the $\operatorname{aBIP}(M, N, P, C, \rho)$ if and only if it is of the form (13.5) for some function $S$ which is a solution of the $\mathbf{a B I P}(M, N, P, C)$.

Proof: Comparing (13.9) and (1.4), we conclude that

$$
\widetilde{G}(z)=\frac{1}{a(z)} G(\sigma(z)) .
$$

The rest follows easily by substituting (13.5) and (13.13) into (13.11) and (13.12) and then using Lemma 13.3.

Making use of Theorem 1.2 we obtain the following description of the set of all solutions of the $\operatorname{aBIP}(M, N, P, C, \rho)$.

Theorem 13.5 Let (13.10) be in force and let $P$ be a nonnegative solution of the LyapunovStein equation (1.8). Then the $\operatorname{aBIP}(M, N, P, C, \rho)$ is solvable and the set of all its solutions is parametrized by the linear fractional transformation

$$
\widetilde{S}(z)=\left(\widetilde{\boldsymbol{\Theta}}_{11}(z) \widetilde{\mathcal{E}}(z)+\widetilde{\boldsymbol{\Theta}}_{12}(z)\right)\left(\widetilde{\boldsymbol{\Theta}}_{21}(z) \widetilde{\mathcal{E}}(z)+\widetilde{\boldsymbol{\Theta}}_{22}(z)\right)^{-1}
$$

based on the meromorphic mvf $\widetilde{\boldsymbol{\Theta}}=\left(\begin{array}{cc}\widetilde{\boldsymbol{\Theta}}_{11} & \widetilde{\boldsymbol{\Theta}}_{12} \\ \widetilde{\boldsymbol{\Theta}}_{21} & \widetilde{\boldsymbol{\Theta}}_{22}\end{array}\right)$, which depends only on the interpolation data (13.8), is rational in $\sigma=b / a$ and is such that the kernel

$$
k_{\widetilde{\boldsymbol{\Theta}}}(z, \omega)=\frac{J-\widetilde{\boldsymbol{\Theta}}(z) J \widetilde{\boldsymbol{\Theta}}(\omega)^{*}}{\rho_{\omega}(z)}
$$

is nonnegative on $\Omega_{+}$. The parameter $\widetilde{\mathcal{E}}$ varies over the set $\mathcal{S}_{\rho}^{p \times q}$ and is of the form

$$
\widetilde{\mathcal{E}}(z)=\left(\begin{array}{cc}
\widehat{\mathcal{E}}(z) & 0 \\
0 & I_{\nu}
\end{array}\right), \quad \widehat{\mathcal{E}} \in \mathcal{S}_{\rho}^{(p-\nu) \times(q-\nu)},
$$

where $\nu$ is the integer defined via (1.11).

Proof: By Theorem 1.2 and in view of Lemma 13.4, all solutions of the $\operatorname{aBIP}(M, N, P, C, \rho)$ are of the form

$$
\widetilde{S}(z)=S(\sigma(z))=\left(\boldsymbol{\Theta}_{11}(\sigma(z)) \mathcal{E}(\sigma(z))+\boldsymbol{\Theta}_{12}(\sigma(z))\right)\left(\boldsymbol{\Theta}_{21}(\sigma(z)) \widetilde{\mathcal{E}}(\sigma(z))+\boldsymbol{\Theta}_{22}(\sigma(z))\right)^{-1},
$$

where $\mathcal{E}$ varies on $\mathcal{S}^{p \times q}$ and is of the form (1.14). Setting

$$
\widetilde{\boldsymbol{\Theta}}(z)=\boldsymbol{\Theta}(\sigma(z)) \text { and } \quad \widehat{\mathcal{E}}(z)=\mathcal{E}(\sigma(z))
$$

in the preceding formula, we come to (13.14). By Lemma 13.2, $\widehat{\mathcal{E}} \in \mathcal{S}_{\rho}^{p \times q}$ whereas the kernel $k_{\widetilde{\Theta}}$ is nonnegative, since $\Theta$ is $J$-inner. 
Remark 13.6 If the set

$$
\{\lambda \in \Omega: a(\lambda)=0 \text { and } b(\lambda) \neq 0\} \cup\{\lambda \in \Omega: a(\lambda) \neq 0 \text { and } b(\lambda)=0\}
$$

is not empty and $M-\zeta N$ is invertible for every point $\zeta \in \mathbb{T}$, then the McMillan degree of the mvf $\widetilde{\boldsymbol{\Theta}}$ exhibited in Theorem 13.5 is equal to $r$, the rank of $P$. A proof may be based on the analogue of formula (7.9) for the present setting and the corollary to Theorem 3.5 of $[?]$.

In much the same way one can obtain the $\rho$-analogue of Theorem 10.1 and describe all the solutions of the $\operatorname{aBIP}(M, N, P, C, \rho)$ in terms of a Redheffer transform.

Theorem 13.7 Let $\Psi_{j k}$ be the functions defined via formulas (10.9)-(10.11) and (10.15) by replacing

$$
G(z)=M-z N \quad \text { by } \quad a(z) M-b(z) N, \quad H(z)=z M^{*}-N^{*} \quad b y \quad b(z) M^{*}-a(z) N^{*}
$$

and by writing $\rho_{\omega}(z)$ and $\delta_{\omega}(z)$ as in (13.1). Then

1. The function $\Psi=\left(\begin{array}{ll}\Psi_{11} & \Psi_{12} \\ \Psi_{21} & \Psi_{22}\end{array}\right)$ is inner in $\Omega_{+}$and belongs to $\mathcal{S}_{\rho}^{(p+q-\nu) \times(p+q-\nu)}$.

2. All the solutions $S$ of the aBIP are parametrized by the Redheffer transformation (10.16), where $\widehat{\mathcal{E}}$ is a free independent parameter varying over $\mathcal{S}_{\rho}^{(p-\nu) \times(q-\nu)}$ and $\nu$ is the integer defined by (1.11).

Theorems 1.2 and 10.1 and their analogues for the open upper half plane $\mathbb{C}_{+}$and the open right half plane $\mathbb{C}_{R}$ as well as more general regions emerge from Theorems 13.5 and 13.7 by making appropriate choices of $a(z)$ and $b(z)$. All the main results of this paper were developed in a notation which is applicable to the more general domain $\Omega_{+}$given in (13.2) by introducing the replacements described in (13.15) and and by writing $\rho_{\omega}(z)$ and $\delta_{\omega}(z)$ as in (13.1). Some indications of how this may done for the classical settings of $\Omega_{+}=\mathbb{C}_{+}$and $\Omega_{+}=\mathbb{C}_{R}$ (under more restrictive assumptions on $P$ and $\Delta_{\omega}(\omega)$ than are imposed here) may be found in [15] and [16], respectively.

\section{References}

[1] D. Alpay and V. Bolotnikov. Two sided Nevanlinna-Pick interpolation for a class of matrix-valued functions. Zeitschrift für Analysis und ihre Anwendungen, 12 (1993), 211-238.

[2] D. Alpay, V. Bolotnikov, A. Dijksma, and H.S.V. de Snoo. On some operator colligations and associated reproducing kernel Hilbert spaces. Operator Theory: Adv. Appl., 61, pages 89-159, 1993. 
[3] D. Alpay and H. Dym. On reproducing kernel spaces, the Schur algorithm and interpolation in a general class of domains, volume 50 of Operator Theory: Advances and Applications, pages 30-77. Birkhäuser Verlag, Basel, 1992.

[4] D. Alpay and H. Dym. On a class of reproducing kernel Hilbert spaces and a new generalization of the Iohvidov's laws. Linear Algebra and its Applications, 178:109-183, 1993.

[5] D. Alpay and H. Dym. On a new class of structured reproducing kernel Hilbert spaces. J. Funct. Anal., 111:1-28, 1993.

[6] D. Arov. Darlington realization of matrix-valued functions. Math. USSR Izvestija, 7:1295-1326, 1973.

[7] D.Z. Arov and M.G. KreĬn. On computations of entropy functionals and their minimums in indeterminate continuation problems. Acta Sci. Math., 45:33-50, 1983.

[8] J. Ball, I. Gohberg, and L. Rodman. Interpolation of rational matrix functions. Birkhäuser Verlag, Basel, 1990.

[9] J. Ball and J.W. Helton. Interpolation problems of Pick-Nevanlinna and Loewner types for meromorphic matrix-functions: parametrization of the set of all solutions. Integral Equations and Operator Theory, 9:155-203, 1986.

[10] P. Bruinsma. Degenerate interpolation problems for Nevanlinna pairs. Indag. Math., N. S., 2(2):179-200, 1991.

[11] V. Dubovoy. Indefinite metric in the interpolation problem of schur for analytic matrix functions IV. Theor. Funktsii, Func. Anal. i Prilozen, 42:46-57, 1984.

[12] V. Dubovoy, B. Fritzsche, and B. Kirstein. Matricial version of the classical Schur problem, volume 129 of Teubner-Texte zur Mathematik. B.G. Teubner Verlagsgesellschaft, Stuttgart-Leipzig, 1992.

[13] H. Dym. J contractive matrix functions, reproducing kernel spaces and interpolation, volume 71 of CBMS Lecture Notes. Amer. Math. Soc., Rhodes Island, 1989.

[14] H. Dym. Shifts, realizations and interpolation, redux, volume 73 of Operator theory: advances and applications, pages 182-243. Birkhäuser Verlag, Basel, 1994.

[15] H. Dym. More on maximum entropy interpolants and maximum determinant completions of associated pick matrices. Integral Equations and Operator Theory, 24:188-229, 1996.

[16] H. Dym. A basic interpolation problem. In D. Sarason S. Axler, J. McCarthy, editor, Holomorphic spaces. Cambridge University press, 1998. 
[17] H. Dym and I. Gohberg. On maximum entropy interpolants and maximum determinant completions of associated pick matrices. Integral Equations and Operator Theory, 23:6188, 1995.

[18] F. R. Gantmacher. The Theory of Matrices, Vol. II. Chelsea Publishing Company, New-York, 1959.

[19] Y. Inouye. Maximum entropy spectral estimation for regular time series of degenerate rank. IEEE Transactions on Acoustics, Speech, and signal Processing, 32(4):733-740, 1984.

[20] V. Katsnelson. Methods of J-theory in continuous interpolation problems of analysis. Private translation of T. Ando, Sapporo, 1982.

[21] V. Katsnelson. Continuous analogues of the Hamburger-Nevanlinna theorem and fundamental matrix inequalities. Amer. Math. Soc. Transl., 136:49-96, 1987.

[22] V. Katsnelson. A transformation of Potapov's fundamental matrix inequality, pages 253-281. Operator Theory: Advances and Applications OT95. Birkhäuser Verlag, Basel, 1997.

[23] V. Katsnelson, A. Kheifets, and P. Yuditskii. An abstract interpolation problem and extension theory of isometric operators, pages 83-96. Operators in spaces of functions and problems in the function theory. Naukova dumka, Kiev, 1987.

[24] V. Katsnelson and B. Kirstein. On the theory of matrix-valued functions belonging to the Smirnov class, pages 299-350. Operator Theory: Advances and Applications OT95. Birkhäuser Verlag, Basel, 1997.

[25] A. Kheifets and P. Yuditskii. An analysis and extension approach of V. P. Potapov's approach to scheme interpolation problems with applications to the generalized bitangential Schur-Nevanlinna-Pick problem and J-inner-outer factorization, pages 133161. Operator Theory: Advances and Applications OT72. Birkhäuser Verlag, Basel, 1994.

[26] I. V. Kovalishina. Analytic theory of a class of interpolation problems. Math. USSR Izv., 22:419- 463, 1984.

[27] I.V. Kovalishina and V.P. Potapov. Seven papers translated from the Russian. American Mathematical Society Translations (2), 138, Providence, R.I., 1988.

[28] A.A. Nudelman. On a generalization of classical interpolation problems. Sov. Math. Dokl., 23:125-128, 1981.

[29] R.A. Penrose. A generalized inverse for matrices. Proceedings Cambridge Phil. Soc., 51:406-413, 1955. 
Department of Theoretical Mathematics

The Weizmann Institute of Science

Rehovot 76100, Israel

MSC: 47A57, 30E05

Submitted: December 15, 1997 